Acta Crystallographica Section A

Foundations of Crystallography

ISSN 0108-7673

Received 22 February 2010

Accepted 3 May 2010

(C) 2010 International Union of Crystallography

Printed in Singapore - all rights reserved

\section{Mode crystallography of distorted structures}

\author{
J. M. Perez-Mato, ${ }^{*}$ D. Orobengoa and M. I. Aroyo
}

Departamento de Física de la Materia Condensada, Facultad de Ciencia y Tecnología, Universidad del País Vasco (UPV-EHU), Apdo 644, 48080 Bilbao, Spain. Correspondence e-mail: jm.perez-mato@ehu.es

The description of displacive distorted structures in terms of symmetry-adapted modes is reviewed. A specific parameterization of the symmetry-mode decomposition of these pseudosymmetric structures defined on the setting of the experimental space group is proposed. This approach closely follows crystallographic conventions and permits a straightforward transformation between symmetry-mode and conventional descriptions of the structures. Multiple examples are presented showing the insight provided by the symmetrymode approach. The methodology is shown at work, illustrating its various possibilities for improving the characterization of distorted structures, for example: detection of hidden structural correlations, identification of fundamental and marginal degrees of freedom, reduction of the effective number of atomic positional parameters, quantitative comparison of structures with the same or different space group, detection of false refinement minima, systematic characterization of thermal behavior, rationalization of phase diagrams and various symmetries in families of compounds etc. The close relation of the symmetry-mode description with the superspace formalism applied to commensurate superstructures is also discussed. Finally, the application of this methodology in the field of ab initio or first-principles calculations is outlined. At present, there are several freely available user-friendly computer tools for performing automatic symmetry-mode analyses. The use of these programs does not require a deep knowledge of group theory and can be applied either a posteriori to analyze a given distorted structure or a priori to parameterize the structure to be determined. It is hoped that this article will encourage the use of these tools. All the examples presented here have been worked out using the program AMPLIMODES [Orobengoa et al. (2009). J. Appl. Cryst. 42, 820-833].

\section{Introduction}

Many crystalline structures can be considered pseudosymmetric with respect to some configuration of higher symmetry. This higher-symmetry arrangement may be another phase of the compound or a virtual reference structure. In the following we will refer to this (real or virtual) structure of higher symmetry as the parent structure or parent phase. By definition, a group-subgroup relation necessarily exists between the space groups of the parent structure and the observed one. This latter can then be qualified as a distorted structure and can be described as the parent crystalline structure plus a static symmetry-breaking structural distortion. If the distortion is sufficiently small, a thermally driven structural transition to the configuration of higher symmetry may take place at higher temperatures (Bruce, 1980; Dove, 1993, 1997; Rabe et $a l ., 2007)$. Ferroic structures are a particular case of this type of distorted structure, with the distorted (ferroic) structure having a lower point group than the parent phase (Wadhawan, 2000). Structural distortions can be of displacive type or may include some type of order-disorder component (symmetrybreaking change of the occupation probabilities of some atomic sites). In the present paper we will only consider purely displacive distorted structures, and the term distorted will be used in this restricted sense. We will see below, however, that some simple order-disorder distortions can also be included within this displacive formalism.

Similarly, as happens with dynamic distortions (thermal vibrations), we know since the development of Landau theory (Landau \& Lifshitz, 1969; Toledano \& Toledano, 1987) that the natural language for dealing with the static frozen distortions present in ferroic structures, and distorted structures in general, is the one of modes. Modes are collective correlated atomic displacements fulfilling specific symmetry properties. Structural distortions in distorted structures can be decomposed into contributions from different modes with symmetries given by irreducible representations of the parent space group. One can then distinguish primary and secondary (induced) distortions with different symmetries, which will have in general quite different weights in the structure, and 
will respond differently to external perturbations. In general, the use of symmetry-adapted modes in the description of distorted structures introduces a natural physical hierarchy among the structural parameters. This can be useful not only for investigating the physical mechanisms that stabilize these phases but also for pure crystallographic purposes. The set of structural parameters used in a mode description of a distorted phase is in general better adapted for a controlled refinement of the structure, or for instance for comparative studies between different materials.

The theoretical basis of symmetry-mode analysis of distorted structures and its practice is well documented (see, for instance, Stokes et al., 1991; Hatch \& Stokes, 2001). But despite its obvious advantages the use of symmetry-adapted modes is still scarce in crystallographic studies of distorted structures. Examples where this approach is applied in a quantitative and systematic form regularly appear in the literature but they represent a minority within the large amount of structural studies of distorted structures. For many years there has been one clear reason for this situation: namely, the mode decomposition analysis in non-trivial cases required a deep knowledge of group theory and a considerable calculation effort for each specific case. In more recent years this situation has changed; free computer programs have been developed allowing fast and automatic symmetry-mode analyses [BasIreps (Rodríguez-Carvajal, 1993), SIMREF (Ritter et al., 1998), SARAh (Wills, 2000), SYMMODES (Capillas et al., 2003), MODY (Sikora et al., 2004)]. These tools have focused on the calculation of a basis of distortion modes relevant in each case. However, this has usually been done within the reference of the parent structure, without using the space-group symmetry of the distorted structure in an explicit form. This has implied in general a formalism and parameterization quite distant from the usual crystallographic description, and has hampered its use among crystallographers.

This situation has improved in recent years with new free software [ISODISPLACE (Campbell et al., 2006) and AMPLIMODES (Orobengoa et al., 2009)]. In particular, we have made available in the Bilbao Crystallographic Server (Aroyo, Kirov et al., 2006, Aroyo, Perez-Mato et al., 2006) a tool (AMPLIMODES) that allows the automatic mode decomposition of any distorted structure (Orobengoa et al., 2009). The program provides, apart from a basis of symmetry modes, their amplitudes in the distorted structure. An important feature of the program is that the parameterization of the structural distortion is carried out in a form that is close to the conventions of crystallography. Modes are given in terms of relative displacements for the asymmetric unit of the distorted phase, such that the actual atomic positions describing the structure in a conventional form are readily obtained from the list of basis modes and their amplitudes. By this means we pretend to introduce a mode parameterization that facilitates a straightforward switch from the symmetrymode approach to the conventional description of a structure, and hopefully will help to standardize and generalize its use in crystallographic studies.
A further step in this direction has been adapting the refinement programs FullProf (Rodríguez-Carvajal, 1993) and AMPLIMODES for their combined use, so that now FullProf can directly use the output of AMPLIMODES and refine distorted structures using as refinable parameters the amplitudes of a basis of symmetry modes. The potential of the symmetry-mode approach for the determination of pseudosymmetric or distorted structures, with the introduction among the structural parameters of a strong hierarchy and the reduction of correlations, has recently been demonstrated for a specific case (Campbell et al., 2007). The automatic combination of these two freely available programs should facilitate and extend the application of the direct symmetry-mode refinement of distorted structures, advancing in the development of a standardized quantitative 'mode crystallography'.

Another important development that has taken place in the last decade is the generalization and extensive use of $a b$ initio density-functional-theory (DFT) calculations and simulations in the investigation of the structure and properties of ferroic materials and distorted structures in general. A systematic use of symmetry-mode considerations would in general be convenient both for the design and analysis of these calculations. It can help both to optimize the calculations and to resolve the different relevant degrees of freedom, distinguishing marginal features from fundamental ones. But, as in the case of the experimental investigations, proper use of symmetry-mode analyses is seldom found in this rapidly growing research field.

Within this context and in the light of the new computer tools mentioned above, we review in this article the symmetrymode approach to the analysis of distorted structures, thoroughly discussing a series of examples. By this means, the virtues and wide possibilities of the analysis of distorted structures in terms of symmetry-adapted modes is illustrated. All the examples presented here have been worked out using the program AMPLIMODES (Orobengoa et al., 2009) and other tools of the Bilbao Crystallographic Server (Aroyo, Kirov et al., 2006, Aroyo, Perez-Mato et al., 2006).

\section{Symmetry-mode description of distorted structures}

We review in this section the basic features of the symmetryadapted mode description of distorted structures, introducing the notation and parameterization employed. In order to simplify the notation, we will avoid when possible any explicit indication of the parameters describing the symmetry properties of the modes (wavevectors, wavevector stars etc.). Thus, we reduce the notation to the basic features which are really needed in a practical case, assuming that we have some computing tool to obtain a valid basis of symmetry-adapted modes, given in the crystallographic format explained below. A more practical introduction with additional examples of the notation used here can be found by Orobengoa et al. (2009). For an extended review of the group theory of displacive modes, see, for instance, Izyumov \& Syromyatnikov (1990) and Stokes et al. (1991). 
The distortion relating a parent structure with the actual displacively distorted structure of lower symmetry can be deconvoluted into two parts:

(i) A set of atomic displacements, which may break some translational symmetry but keep the metrics of the underlying parent lattice, so that the basis vectors of the resulting new Bravais lattice are exactly given by some integer combination of the primitive unit-cell basis vectors of the parent structure.

(ii) A strain of the parent lattice mentioned in (i).

This separation into two parts corresponds to the distinction of the elastic degrees of freedom from the internal atomic degrees of freedom in the structure, and is done automatically if the atomic displacements and positions are expressed in relative coordinates with respect to the cell parameters, as usually done in crystallography.

In general, for full consistency and formal rigor (orthogonality properties of modes etc.), the mode analysis should be performed assuming that the relative coordinates of the distorted structure correspond to step (i) above, i.e. to a structure with an unstrained lattice, so that its unit cell, in general a supercell, perfectly matches that of the parent structure. The additional strain present in the real structure can be added automatically a posteriori by taking the real unit cell while keeping the same relative coordinates.

Let $\mathbf{r}_{0}(\mu)$ be the positions of the atoms $\mu(\mu=1, \ldots, s)$ within an asymmetric unit of the parent structure with space group $\mathcal{G}$. The asymmetric unit of the observed distorted structure with lower space group $\mathcal{H}$, subgroup of $\mathcal{G}$, will in general have a larger number of atoms owing to the splitting of the Wyckoff orbits of the higher-symmetry space group (Wondratschek, 1993). Its atomic positions can then in general be expressed as

$$
\mathbf{r}(\mu, i)=\mathbf{r}_{0}(\mu, i)+\mathbf{u}(\mu, i)
$$

where $\mathbf{r}_{0}(\mu, i)\left(\mu=1, \ldots, s ; i=1, \ldots, n_{\mu}\right)$ are the corresponding atomic positions in the parent structure, expressed in the setting of the low-symmetry space group, with the index $i$ enumerating the formally split atomic orbits coming from a single Wyckoff orbit in $\mathcal{G}$.

The set of atomic displacements $\mathbf{u}(\mu, i)$ within an asymmetric unit of the distorted structure, with symmetry given by space group $\mathcal{H}$, fully defines the displacive distortion relating both structures. In general, it can be expressed as a linear combination of the contributions of a basis of symmetryadapted modes,

$$
\mathbf{u}(\mu, i)=\sum_{\tau, m} A_{\tau, m} \boldsymbol{\varepsilon}(\tau, m \mid \mu, i) .
$$

The indices $\tau$ and $m$ label all possible distinct allowed symmetry-adapted distortion modes in the chosen basis. In short, we shall call these modes basis modes. $\tau$ stands for the possible different mode symmetries, while $m\left(m=1, \ldots, n_{\tau}\right)$ enumerates the possible different independent modes for a given symmetry. The contribution of each mode is separated into an amplitude $A_{\tau, m}$ and a set of atomic displacements $\boldsymbol{\varepsilon}$ normalized within a primitive unit cell, both being real quantities. The atomic displacements $\boldsymbol{\varepsilon}$ for a given basis mode $\boldsymbol{\varepsilon}(\tau, m)$ form its so-called polarization vector, that describes the corresponding set of correlated relative atomic displacements. The set of displacements $\boldsymbol{\varepsilon}(\tau, m \mid \mu, i)$ within the asymmetric unit of the $\mathcal{H}$ structure, i.e. with $\mu=1, \ldots, s, i=$ $1, \ldots, n_{\mu}$, define unambiguously the polarization vector of the corresponding symmetry mode $(\tau, m)$. The displacements of the remaining atoms within the $\mathcal{H}$ unit cell are obtained by the symmetry operations of the space group $\mathcal{H}$ that relate these atoms with those in the asymmetric unit. By definition, each of the modes in equation (2) separately maintains at least the symmetry given by the space group $\mathcal{H}$. Therefore, the displacement of an atom related by an operation $(\mathbf{R}, \mathbf{t})$ of $\mathcal{H}$ with atom $(\mu, i)$ in the asymmetric unit will be given by $\boldsymbol{R} \boldsymbol{\varepsilon}(\tau, m \mid \mu, i)$. The polarization vector $\boldsymbol{\varepsilon}(\tau, m)$ defines the symmetry-adapted basis mode $(\tau, m)$ except for an arbitrary amplitude; therefore we will use in the following the terms mode and mode polarization vector as essentially synonymous.

We choose the polarization vectors of the basis modes in (2) normalized within a primitive unit cell of the $\mathcal{H}$ lattice, i.e.

$$
\sum_{\mu, i} \operatorname{mult}(\mu, i)|\boldsymbol{\varepsilon}(\tau, m \mid \mu, i)|^{2}=1
$$

The symmetry relation mentioned above permits the reduction of the sum in (3) to the asymmetric unit by considering the multiplicity $\operatorname{mult}(\mu, i)$ (the multiplicity within a primitive unit cell for the space group $\mathcal{H}$ ) of the corresponding Wyckoff positions. It is important that the normalization in (3) is performed with the mode displacements expressed in an absolute length scale. In addition, the basis modes introduced in (2) are chosen orthogonal, so that their polarization vectors fulfill

$$
\sum_{\mu, i} \operatorname{mult}(\mu, i) \boldsymbol{\varepsilon}(\tau, m \mid \mu, i) \cdot \boldsymbol{\varepsilon}\left(\tau^{\prime}, m^{\prime} \mid \mu, i\right)=\delta_{\tau \tau^{\prime}} \delta_{m m^{\prime}} .
$$

This orthogonality is automatically satisfied by modes of different symmetry, while in the case of modes of the same symmetry a systematic orthogonalization procedure can be applied. Note that this implies that the set of symmetryadapted modes is not unique and a certain arbitrary choice must be made for any practical calculation.

The displacements of the atoms in each Wyckoff orbit of the parent structure form an invariant subspace within the space of the structural distortions for all symmetry operations, so that the symmetry-adapted basis modes can be chosen considering separate basis modes for each atomic Wyckoff orbit of the parent structure, i.e. $\boldsymbol{\varepsilon}(\tau, m \mid \mu, i)=0$ for all $\mu$ except a specific one. In addition, the symmetry constraints of the polarization vector of a given mode only depend on the type of Wyckoff orbit, so that the polarization vectors of the basis modes can be chosen identical for all orbits corresponding to the same Wyckoff position. Hence, the index $m$ in the mode basis $\{\boldsymbol{\varepsilon}(\tau, m)\}$ enumerating the modes associated with the same symmetry can be decomposed into two labels: one indicating the atom representative $\mu$ of the set of the parent-symmetry-related atoms having displacements for this mode, and an additional index for further enumeration of the modes of the same symmetry and the same atoms. We will 
maintain, however, for simplicity whenever possible a single label $m$ as a short symbolic notation.

The maximum number of modes that can be included in (2) coincides with the number of free atomic positional parameters necessary to describe the $\mathcal{H}$ structure in a conventional form, i.e. with the number of $(\mathcal{H}$-symmetry allowed) free parameters in the set of atomic displacements $\{\mathbf{u}(\mu, i)\}$. Expression (2) is then in fact a change of basis in the mathematical vector space of structural parameters describing the structural distortion, i.e. a linear transformation between the atomic positional parameters $\{\mathbf{u}(\mu, i)\}$ that define the atomic positions in the $\mathcal{H}$ structure and the amplitudes $\left\{A_{\tau, m}\right\}$ of the chosen basis of symmetry-adapted modes. It is important to stress that the dimension of these amplitudes $A_{\tau, m}$ is length. Therefore, they can be expressed for instance in angstroms and the magnitude of different distortion modes present in a distorted structure can be directly compared even if they represent collective atomic displacements of very different kinds. The values of the amplitudes $A_{\tau, m}$ can be readily obtained from the set of atomic displacements $\{\mathbf{u}(\mu, i)\}$ once the basis of symmetry-adapted modes $\boldsymbol{\varepsilon}(\tau, m)$ has been chosen. Using their orthonormality properties, a simple scalar product of the distortion with each normalized mode of the basis provides the corresponding amplitude,

$$
A_{\tau, m}=\sum_{\mu, i} \operatorname{mult}(\mu, i) \boldsymbol{\varepsilon}(\tau, m \mid \mu, i) \cdot \mathbf{u}(\mu, i) .
$$

The symmetry of each basis mode $\boldsymbol{\varepsilon}$ is characterized by an irreducible representation (irrep) of the space group $\mathcal{G}$, defining its transformation properties for the operations of the high-symmetry group $\mathcal{G}$, plus generally some additional restrictions such that the mode keeps the observed symmetry $\mathcal{H}$. In general each basis mode in (2) maintains in the structure a symmetry that is intermediate between $\mathcal{G}$ and $\mathcal{H}$ (including $\mathcal{H}$ and $\mathcal{G}$ themselves). In other words, its isotropy subgroup (Jaric \& Senechal, 1984; Hatch \& Stokes, 1985) is in general a supergroup of $\mathcal{H}$. This implies that the basis modes in (2) are normally restricted to a specific subspace within the representation space associated with their irrep. As this additional restriction is always present if we are working with a specific space group $\mathcal{H}$, the irrep $\tau$ associated with the mode can be used as a single label for describing its symmetry, with the additional restriction (forced by the invariance with respect to the space group $\mathcal{H}$ ) left implicit.

The distortion modes with isotropy group equal to $\mathcal{H}$ can be called primary, while those with isotropy groups given by subgroups of $\mathcal{G}$ which are distinct supergroups of $\mathcal{H}$ are usually termed secondary. A primary distortion mode is sufficient to produce the observed symmetry break between the parent and the observed structure, while secondary distortion modes alone would yield a higher symmetry. Trivial examples of secondary distortion modes are those that maintain the symmetry of the parent structure, i.e. they transform according to the identity irrep. This type of secondary symmetry mode always exists except if all the atoms in the parent structure are located in special positions with all their coordinates forced to special values.
The determination of the amplitudes $A_{\tau, m}$ of the symmetrybreaking modes does not require knowing a specific 'real' parent structure. Only the amplitudes of modes transforming according to the identity irrep, i.e. those that do not break the space group and are therefore already allowed in the parent structure, depend on the specific atomic coordinates of the parent structure. A change in the atomic coordinates that are variable under space group $\mathcal{G}$ only introduces additional atomic displacements described by modes that transform according to the identity irrep. Therefore, the rest of the distortion, namely the symmetry-breaking distortion, remains unchanged for any values of these variable coordinates. Hence, the calculation of the amplitudes $A_{\tau, m}$ of the symmetry-breaking distortion modes only requires a minimal knowledge from the high-symmetry structure, namely the set of atoms in its asymmetric unit, their type of Wyckoff position and their correspondence with the atoms in the distorted structure. This association between the atoms in the high- and low-symmetry phases is necessary for the calculation of the set of displacements $\mathbf{u}(\mu, i)$, but for this purpose a rough approximate guess of the crystallographic free coordinates of the atoms in the parent structure is in general sufficient.

Some ambiguity in the results of the mode decomposition occurs if the distorted phase is polar. The set of atomic displacements relating the parent and the distorted structure includes in general a global translation of the crystal that depends on the (arbitrary) choice of origin of the polar structure. A convenient origin choice is the one that makes this global translation zero. This is the choice made in all analyses of polar structures discussed in this article.

It is also very convenient to express the total distortion as a combination of a single distortion mode for each of the allowed irreps,

$$
\mathbf{u}(\mu, i)=\sum_{\tau} A_{\tau} \mathbf{e}(\tau \mid \mu, i)
$$

The amplitudes $A_{\tau}$ are given by $\left(\sum_{m} A_{\tau, m}^{2}\right)^{1 / 2}$, while the corresponding normalized polarization vector $\mathbf{e}(\tau)$ is determined by the linear combination of the basis modes $\boldsymbol{\varepsilon}(\tau, m)$, with fixed $\tau$, realized in the structure

$$
\mathbf{e}(\tau \mid \mu, i)=\sum_{m} a_{\tau, m} \boldsymbol{\varepsilon}(\tau, m \mid \mu, i)
$$

with

$$
a_{\tau, m}=A_{\tau, m} /\left(\sum_{m} A_{\tau, m}^{2}\right)^{1 / 2}
$$

The distortion mode of symmetry $\tau$ present in the structure can therefore be described by a global amplitude $A_{\tau}$ and an $n_{\tau^{-}}$ dimensional normalized polarization vector with components $\left\{a_{\tau, m}\right\}$ in the working basis $\{\boldsymbol{\varepsilon}(\tau, m)\}$. The components $\left\{a_{\tau, m}\right\}$ define the direction taken by the observed distortion in the $n_{\tau^{-}}$ dimensional space of allowed $\tau$ distortions. The set of amplitudes $\left\{A_{\tau, m}\right\}$ are indeed the components of an $n_{\tau}$-dimensional vector expressed in an orthonormal basis, and any possible distortion $\tau$ of the parent structure can be expressed by an amplitude $A_{\tau}$ and the normalized vector $\left\{a_{\tau, m}\right\}$. 
Table 1

Asymmetric unit of the $A m m 2$ structure of $\mathrm{BaTiO}_{3}$ at $190 \mathrm{~K}$ according to Kwei et al. (1993), compared with the reference structure, i.e. the $P m \overline{3} m$ parent structure expressed in the same setting.

The unit cell of the reference structure is used for translating the atomic displacements into absolute values. Note the splitting of the oxygen orbit in the orthorhombic space group. The origin of the published Amm2 structure has been shifted along $z$ so that the atomic displacements relating both structures do not include a global translation.

\begin{tabular}{|c|c|c|c|c|c|c|c|}
\hline \multicolumn{5}{|c|}{$\begin{array}{l}\text { Amm } 2 \text { phase } \\
(a=3.9828, b=5.6745, c=5.6916 \AA)\end{array}$} & \multicolumn{3}{|c|}{$\begin{array}{l}P m \overline{3} m \text { phase in } A m m 2 \text { setting } \\
\text { (reference structure) } \\
(a=4.006, b=5.665, c=5.665 \AA)\end{array}$} \\
\hline Atom & $\begin{array}{l}\text { Wyckoff } \\
\text { position }\end{array}$ & $x$ & $y$ & $z$ & $x$ & $y$ & $z$ \\
\hline $\mathrm{Ba}$ & $2 a$ & 0.0 & 0.0 & 0.00508 & 0.0 & 0.0 & 0.0 \\
\hline $\mathrm{Ti}$ & $2 b$ & 0.5 & 0.0 & $0.5221(5)$ & 0.5 & 0.0 & 0.5 \\
\hline O1_1 & $4 e$ & 0.5 & $0.2561(3)$ & $0.2394(4)$ & 0.5 & 0.25 & 0.25 \\
\hline O1_2 & $2 a$ & 0.0 & 0.0 & $0.4941(6)$ & 0.0 & 0.0 & 0.5 \\
\hline
\end{tabular}

While the polarization vectors $\boldsymbol{\varepsilon}(\tau, m)$ of the symmetryadapted basis only depend on the symmetry properties of the irrep $\tau$ and include some arbitrary choice, the polarization vectors $\mathbf{e}(\tau)$ describing the $\tau$ distortion are specific for each concrete structure. We shall call these system-dependent symmetry-adapted global distortion modes, present in the distorted structure, irrep distortion components, or simply irrep distortions, to be distinguished from the irrep basis modes $\boldsymbol{\varepsilon}(\tau, m)$. Their amplitudes $A_{\tau}$ define the global amplitude of each irrep distortion in the structure and will vary with external perturbations or with changes of the thermodynamic variables of the system. On the other hand, the corresponding polarization vectors $\mathbf{e}(\tau)$ are expected to be weakly dependent with respect to external fields, and among isomorphic materials. In many cases they can be related to low-energy static normal modes of the system (see $\S 4$ and $\S 11$ ) that characterize not only the free-energy minimum realized by the observed phase but also the low-energy arrangements around this minimum where the system may move with relatively low energy cost.

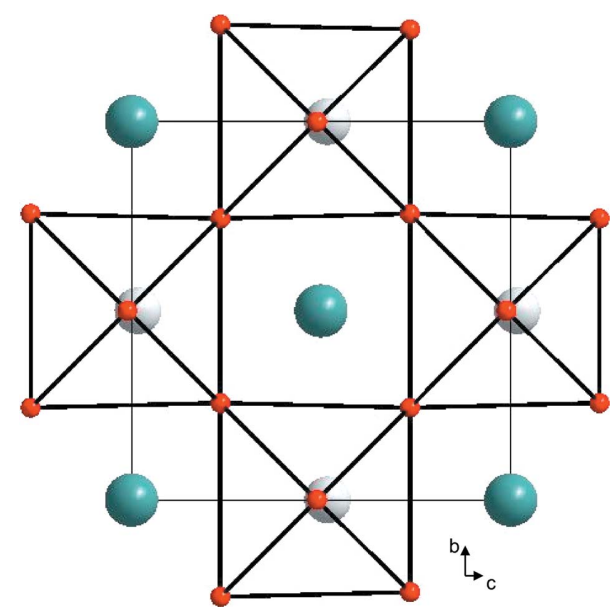

Figure 1

Projection along the $x$ axis of the $A m m 2$ structure of $\mathrm{BaTiO}_{3}$ at $190 \mathrm{~K}$, according to Kwei et al. (1993).
A quantitative assessment of the similitude of the polarization vectors of the irrep distortions of the same symmetry present in different structures can be readily made by calculating their scalar product. If the two distortions are expressed by their components $\left\{a_{\tau, m}\right\}$ and $\left\{a_{\tau, m}^{\prime}\right\}$, using equivalent bases, then this scalar product is just $\sum_{m} a_{\tau, m} a_{\tau, m}^{\prime}$.

\section{Example of the orthorhombic ferroelectric phase of $\mathrm{BaTiO}_{3}$}

Barium titanate, one of the most studied ferroelectrics, is known to have a parent phase having $\operatorname{Pm} \overline{3} m$ symmetry and three consecutive ferroelectric phases of different symmetries as temperature is lowered (Lines \& Glass, 1977). In particular, it has an intermediate orthorhombic phase with space group Amm2 in the temperature interval [183 K, $273 \mathrm{~K}$ ] (Tomaszewski, 1992), which we consider here as a first example.

Without including the orthorhombic strain, the space group $A m m 2$ of the orthorhombic phase of $\mathrm{BaTiO}_{3}$ is related to that of its cubic phase by the transformation $(\mathbf{c}, \mathbf{a}-\mathbf{b}, \mathbf{a}+\mathbf{b}$; $0,0,0) .{ }^{\mathbf{1}}$ The reported structure (Kwei et al., 1993) of this orthorhombic phase of $\mathrm{BaTiO}_{3}$ is reproduced in Table 1 and shown in Fig. 1. The maximum atomic displacement in the distortion with respect to the cubic perovskite phase is smaller than $0.13 \AA$. The number of atomic positional parameters in the structure is five, but, as the structure is polar along $z$, only four of them are really independent, owing to the arbitrariness of the origin along $z$. Before performing the mode decomposition, as discussed in the previous section, we shift the origin of the published Amm2 structure along the polar direction so that the atomic displacements relating both structures do not include a global translation. This shift has already been made in the structure given in Table 1 . The atomic displacements relating the $A m m 2$ structure with the parent perovskite structure are then readily obtained from the comparison of the asymmetric unit of the Amm2 structure with that of the cubic parent phase expressed in the same setting (the reference structure), which is also listed in Table 1.

The Amm 2 distortion decomposes into two distortion modes of different symmetry corresponding to the irreps GM4- and GM5-. ${ }^{2}$ Both irreps have Amm2 as isotropy subgroup. The space of the GM4- distortion is fourdimensional while the GM5- distortion subspace is onedimensional $\left(n_{\tau}=4\right.$ and 1 for GM4- and GM5-, respectively). A basis of five symmetry-adapted modes $\boldsymbol{\varepsilon}$ is listed in Table 2. The polarization vectors of the GM4- modes for Ba and $\mathrm{Ti}$ are equal and correspond to $z$ displacements of $1 \AA$,

\footnotetext{
${ }^{1}$ Throughout the paper the transformation between the conventional settings of the high- and low-symmetry space groups will be given in this form, where the first part indicates the basis vectors $\mathbf{a}^{\prime}, \mathbf{b}^{\prime}, \mathbf{c}^{\prime}$ of the subgroup in terms of those of the supergroup $\mathbf{a}, \mathbf{b}, \mathbf{c}$, while the second part defines its origin shift in the $(\mathbf{a}, \mathbf{b}, \mathbf{c})$ basis.

${ }^{2}$ We follow in the whole article the irrep notation of ISOTROPY (Stokes \& Hatch, 2002), which is essentially that of Cracknell et al. (1979). The irreps at the $\Gamma$ point will be labeled with the symbol GM, instead of the Greek letter, to simplify the notation.
} 
Table 2

Basis of symmetry modes of the parent structure $\operatorname{Pm} \overline{3} m$ of $\mathrm{BaTiO}_{3}$, restricted to the isotropy subgroup $A m m 2$.

The atomic displacements for the polarization vector of each mode are expressed in relative units with respect to the reference unit cell, indicating only the displacements of the Amm2 asymmetric unit (see Table 1 ). Modes are normalized within a primitive unit cell of the $A \mathrm{~mm} 2$ structure. The modes are labeled by their irrep, the atom representative of the parent Wyckoff orbit involved in the mode and an additional numerical index in the case of the existence of several independent modes for the same irrep and the same atom.

\begin{tabular}{lllcr}
\hline Mode & & $\delta x$ & $\delta y$ & $\delta z$ \\
\hline GM4-, Ba & Ba & 0.000000 & 0.000000 & 0.176512 \\
GM4-, Ti & Ti & 0.000000 & 0.000000 & 0.176512 \\
GM4-, O1, 1 & O1_1 & 0.000000 & 0.062406 & 0.062406 \\
& O1_2 & 0.000000 & 0.000000 & 0.124813 \\
GM4-, O1, 2 & O1_1 & 0.000000 & -0.088256 & 0.088256 \\
& O1_2 & 0.000000 & 0.000000 & 0.000000 \\
GM5-, O1 & O1_1 & 0.00000 & -0.062406 & -0.062406 \\
& O1_2 & 0.00000 & 0.00000 & 0.124813 \\
\hline
\end{tabular}

while for the $\mathrm{O}$ atoms there are two independent GM4modes that can be chosen as shown in Table 2. The first of these two oxygen modes involves displacements (in angstroms) of $(0,1 / \sqrt{8}, 1 / \sqrt{8})$ for O1_1 and $(0,0,1 / \sqrt{2})$ for O1_2, while for the second one only O_1 in the asymmetric unit has a non-zero displacement given by $(0,-1 / 2,1 / 2)$. Note that the modes for $\mathrm{Ba}$ and $\mathrm{Ti}$ are described by a single threedimensional vector as their orbit is not split by the symmetry break, while in the case of the $\mathrm{O}$ atoms the basis modes are given, in general, by two three-dimensional vectors, one for each oxygen, as they merge into a single orbit in the parent symmetry. The modes in Table 2 are expressed in relative units with respect to the unit cell of the reference structure for practical purposes.

The amplitudes for the five modes listed in Table 2 can be calculated using equation (5). The global amplitudes of the GM4 - and GM5 - distortions result to be 0.165 (3) $\AA$ and 0.006 (3) $\AA$, respectively [see Orobengoa et al. (2009) for more details of the calculation]. These amplitudes can be obtained from the data in Tables 1 and 2. Substracting the reference and the experimental structures of Table 1, the atomic displacements are derived. Calculating their scalar product (in absolute units) with the basis modes of Table 2, as indicated in equation (5), the amplitudes of the basis modes are obtained, and from them the amplitudes of the normalized polarization vectors can be immediately derived [see equation (7)]. The distortion GM5- is therefore more than 25 times smaller than the distortion GM4-, which is the polar one, responsible for the macroscopic polarization. Fig. 2(a) depicts the polarization vector of the distortion mode GM5-, which is fully determined by symmetry and is listed in Table 2 . It is a non-polar mode, totally alien to the ferroelectric instability. Its much smaller weight in the structure is fully consistent with the physical origin of this phase. In fact, the amplitude of mode GM5 - is so small that its contribution to the actual values of the atomic positions is very close to their standard deviations.
Table 3

Polarization vector (normalized to $1 \AA$ ) of the polar GM4- distortion mode present in the orthorhombic phase of $\mathrm{BaTiO}_{3}$.

The mode is defined using the asymmetric unit of the reference structure and unit cell indicated in Table 1. Displacements are expressed in relative units. This polarization vector corresponds to the combination of the four GM4basis vectors described in Table 2 with amplitudes $0.17,0.76,-0.25$ and -0.57 (in the same order as in Table 2).

\begin{tabular}{lllr}
\hline Atom & $\delta x$ & $\delta y$ & \multicolumn{1}{c}{$\delta z$} \\
\hline Ba & 0.0000 & 0.0000 & 0.0308 \\
Ti & 0.0000 & 0.0000 & 0.1339 \\
O1_1 & 0.0000 & 0.0349 & -0.0665 \\
O1_2 & 0.0000 & 0.0000 & -0.0317 \\
\hline
\end{tabular}

The extremely small value of the GM5- distortion implies that the structure has some 'hidden' non-crystallographic approximate correlation among its atomic coordinates. This can be clearly seen by inspecting in Table 3 the polarization vector of the GM4- distortion mode present in the structure. The five non-zero displacement components in this table are not independent but are related by three relations: (i) the absence of global translation, (ii) normalization and (iii) GM4- symmetry. More specifically, the GM4- character of the mode forces the following relation among the components of the oxygen displacements: $\delta y_{\mathrm{O} 1}+\delta z_{\mathrm{O} 1}-\delta z_{\mathrm{O} 2}=0$. These

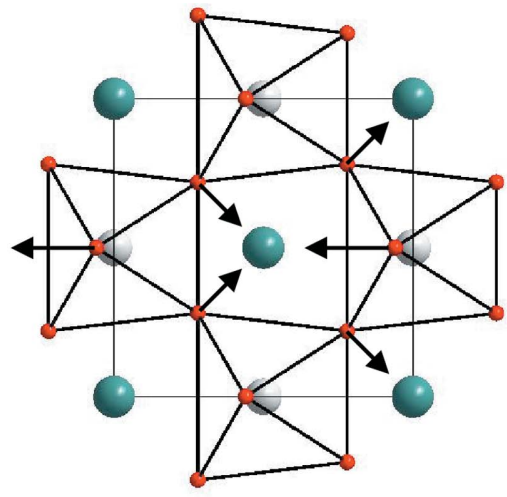

(a)

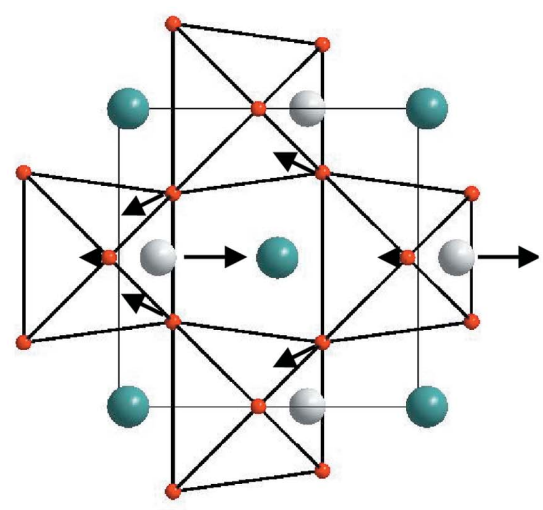

(b)

Figure 2

Scheme of the polarization vectors (projected on the plane $y z$ ) of the distortions GM5- $(a)$ and GM4- (b) present in the Amm2 structure of $\mathrm{BaTiO}_{3}$. The figure shows in each case the distorted structure for an exaggerated amplitude of the mode. Also, schematic arrows indicating the atomic displacements are depicted within a single unit cell. 
three relations reduce the number of adjustable free parameters of the GM4- polarization vector to two, which with its amplitude and the single parameter describing the GM5distortion make the expected total of four degrees of freedom in the structure. As the GM5- distortion mode is very small, the GM4- symmetry is fulfilled to a good approximation by the total distortion, so that the experimental coordinates of the $\mathrm{O}$ atoms satisfy $y_{\mathrm{O} 1}+z_{\mathrm{O} 1}-z_{\mathrm{O} 2} \simeq 0$ (for the reported structure $y_{\mathrm{O} 1}+z_{\mathrm{O} 1}-z_{\mathrm{O} 2}=0.0014 \pm 0.0008$ ). This is a nontrivial non-crystallographic approximate correlation, which is a direct signature of the physical mechanism responsible for the stabilization of this phase, namely the thermal instability of a GM4- polar mode.

The polar GM4- distortion mode present in the Amm2 structure of $\mathrm{BaTiO}_{3}$ is depicted in Fig. 2(b). Although the distortion associated with this phase is usually described as a simple change of the spontaneous polarization (order parameter) from the direction $(1,0,0)$ in the tetragonal phase to the $(1,1,0)$ direction in this orthorhombic one (Lines \& Glass, 1977), it is remarkable that the scheme of correlated atomic displacements is rather complex, and their relation with those associated with the tetragonal ferroelectric phase are not obvious. However, indeed the GM4- distortion shown in Table 3 and Fig. 2(b) is closely connected with the simple polar distortion along a tetragonal axis present in the tetragonal phase. A quantitative comparison of both distortions can be made if they are considered in the common reference of the parent phase.

Note that in this example both irrep distortions could be considered primary from the symmetry viewpoint, as both have as isotropy or invariance subgroup the observed space group. From a structural viewpoint it is, however, the comparison of the amplitudes of the two irrep distortions in the experimental structure that shows the primary role played in the stabilization of the phase of one of them.

\section{Hierarchy of modes}

The large difference in amplitude of the two distortion modes of different symmetry present in the Amm2 structure of $\mathrm{BaTiO}_{3}$ is a simple example of a property that happens rather systematically in all kinds of distorted structures. If a structure is pseudosymmetric, the minimum of the free energy corresponding to this phase within the configuration space of the system should be located in the proximity of a saddle point corresponding to the higher-symmetry configuration. The closeness of both points allows in general a description of this energy minimum by a truncated Taylor expansion around the saddle point associated with the high-symmetry configuration. This Taylor expansion expressed in terms of the amplitudes of normal static distortion modes, i.e. diagonal for the secondorder terms of the expansion, is the starting point of the Landau theory of structural phase transitions (Landau \& Lifshitz, 1969). This topological property of the energy landscape around distorted structures is, however, rather general, and can be used to characterize the structural properties of a distorted structure, independent of the existence or not of a phase transition.

The first terms of a Landau-type expansion around the unstable high-symmetry configuration, with space group $\mathcal{G}$ and close to the distorted phase with space group $\mathcal{H}$ (subgroup of $\mathcal{G})$, can be written as

$$
E=E_{0}+\sum \beta_{m} \rho_{\mathrm{GM} 1, m}+\frac{1}{2} \sum \kappa_{\tau, n}\left(\sum_{j} \rho_{\tau, n, j}^{2}\right)+\ldots,
$$

where the mode amplitudes $\rho_{\tau, n, j}$ in (8) correspond to all displacive normal modes. These are classified according to their irrep $\tau$ (of $\mathcal{G}$ ), a multiplicity label $n$, and a third index $j$ for enumerating the different degenerate modes associated with the same irrep if this latter is multidimensional, so that several modes (same stiffness coefficient $\kappa_{\tau, n}$ ) exist for the same irrep. All symmetry-breaking modes, i.e. all modes not transforming according to the identity representation, do not have linear terms in the energy expansion (8) (the energy of a $\mathcal{G}$ configuration is necessarily extremal with respect to $\mathcal{G}$ symmetrybreaking distortions). The linear terms in (8) are therefore reduced to the distortion modes allowed in the space group $\mathcal{G}$, i.e. those transforming according to identity irrep GM1, and therefore allowed to be non-zero in the $\mathcal{G}$ configuration.

We choose the normal-mode amplitudes $\rho_{\tau, n, j}$ in (8) real, and they refer to modes that, apart from being symmetryadapted, are also eigenmodes of the matrix of second derivatives of the free energy with respect to the atomic displacements. We can say that within the existing freedom in the choice of a symmetry-adapted basis the set of normal modes corresponding to the amplitudes $\rho_{\tau, n, j}$ is a specific choice that apart from being a symmetry-adapted is also a physically adapted basis. These normal modes decompose the space of structural degrees of freedom into collective modes that are energetically independent in the harmonic approximation, their stiffness coefficients $\kappa_{\tau, n}$ being a measure of their energy cost. They are eigenmodes of the matrix of atomic force constants. To distinguish this privileged basis of symmetryadapted modes, we shall call them eigenmodes.

At least one of the stiffness coefficients $\kappa_{\tau, n}$ in (8) must be negative to make the high-symmetry configuration unstable. The anharmonic terms of lowest order, subsequent to those shown in (8), are then sufficient to explain the off-center minima corresponding to the distorted structure. This implies in general that the observed structural distortion corresponding to these off-center minima will contain mainly low-energy eigenmodes. Among them one can distinguish the contribution of primary and secondary eigenmodes, but this distinction includes now a physical condition to the considerations in $\$ 2$, where only symmetry properties were considered.

Primary eigenmodes. Primary eigenmodes are in general those for which their condensation is sufficient to explain the observed symmetry break between the parent and the observed phase and are intrinsically unstable (their stiffness coefficient is negative), while secondary eigenmodes are those that are only present as an induced effect. Within this viewpoint, primary and secondary eigenmodes can be of the same 
symmetry, their difference being their intrinsic instability or stability (see example in previous section).

Secondary eigenmodes. Secondary eigenmodes, despite having in general positive stiffness coefficients and hence being hard modes, appear in the total distortion because they have a symmetry-allowed anharmonic coupling with the primary ones of type $\rho_{\mathrm{s}} P^{(m)}\left(\rho_{\mathrm{p} 1}, \ldots, \rho_{\mathrm{p} n}\right)$ where $P^{(m)}$ is a polynomial term of order $m$ in the amplitudes of the primary eigenmodes $\left(\rho_{\mathrm{p} 1}, \ldots, \rho_{\mathrm{p} n}\right)$. The minimal allowed order $m$ has been called the faintness index (Aizu, 1974) of the corresponding secondary mode. Neglecting higher-order terms, this lowest coupling is sufficient for producing a non-zero amplitude of a secondary mode at the energy minimum, if the primary distortions are non-zero,

$$
\rho_{\mathrm{s}} \simeq\left(1 / \kappa_{\mathrm{s}}\right) P^{(m)}\left(\rho_{\mathrm{p} 1}, \ldots, \rho_{\mathrm{p} n}\right),
$$

where $\kappa_{\mathrm{s}}$ is the stiffness coefficient of the mode amplitude $\rho_{\mathrm{s}}$ in (8). All secondary eigenmodes present in the distorted phase of space group $\mathcal{H}$ are necessarily coupled with the primary ones with terms of this type, i.e. linear in the amplitude of the secondary eigenmode. Any eigenmode having as isotropy group or invariance group a subgroup of $\mathcal{G}$ which is a supergroup of $\mathcal{H}$ has such coupling terms, and is allowed in the distorted phase, in accordance with Curie laws (Authier, 2003). Thus this type of coupling is a necessary and sufficient condition for a mode to be present in the distorted structure. Within this perspective, the space-group symmetry $\mathcal{H}$ associated with the distorted structure is just an efficient form of defining and introducing the symmetry restrictions that all eigenmodes condensed in the distorted phase should fulfill.

The eigenmodes $\left\{\mathbf{e}_{\mathrm{E}}(\tau, n)\right\}$ compatible with the symmetry $\mathcal{H}$ of the distorted phase can be labeled in the same way as we did with a general symmetry-adapted basis for an $\mathcal{H}$ distortion in (2), and we can express them in terms of the chosen symmetryadapted basis,

$$
\mathbf{e}_{\mathrm{E}}(\tau, n \mid \mu, i)=\sum_{m} b_{\tau, m}^{(n)} \boldsymbol{\varepsilon}(\tau, m \mid \mu, i), \quad n=1, \ldots, n_{\tau} .
$$

In a shorter vector notation,

$$
\mathbf{e}_{\mathrm{E}}(\tau, n)=\sum_{m} b_{\tau, m}^{(n)} \boldsymbol{\varepsilon}(\tau, m), \quad n=1, \ldots, n_{\tau}
$$

or

$$
\mathbf{e}_{\mathrm{E}}(\tau, n)=\left(b_{\tau, 1}^{(n)}, b_{\tau, 2}^{(n)}, \ldots, b_{\tau, n_{\tau}}^{(n)}\right) .
$$

The eigenmodes $\left\{\mathbf{e}_{\mathrm{E}}(\tau, n)\right\}$ can be used as a privileged symmetry and physically adapted basis to describe the irrep distortions $\{e(\tau)\}$ [see equation (6)] present in a distorted structure,

$$
\mathbf{e}(\tau)=\sum_{n} a_{\tau, n}^{\mathrm{E}} \mathbf{e}_{\mathrm{E}}(\tau, n)
$$

According to the arguments above, if $\tau$ is the symmetry of a primary mode the decomposition (11) will be dominated by the unstable primary eigenmodes within the set of eigenmodes $\mathbf{e}_{\mathrm{E}}(\tau, n)$ of the same symmetry $\tau$. The rest of the hard eigenmodes of the same symmetry $\tau$ will contribute in general with much smaller amplitudes, described in a first approximation by equations of type (9). As can be seen in (9), secondary eigenmodes with larger stiffness constants are expected to have smaller amplitudes in the distortion, although the strength of the coupling with the primary eigenmodes also plays a role and may alter this general trend.

If $\tau$ corresponds to a symmetry only associated with secondary eigenmodes, the static distortion of this symmetry present in the structure is expected to be much smaller than the primary one, because of its typical dependence on a power of the primary-mode amplitudes. The relative weight of the different eigenmodes of the same symmetry will be essentially governed by (9), i.e. their relative amplitudes are approximately inversely proportional to their stiffness, and proportional to their coupling with the primary modes.

Summarizing, the decomposition of a distorted structure in terms of symmetry modes is expected to show quite different amplitudes for the different irrep distortions present in the structure. Distortion modes that are primary from the symmetry viewpoint will have larger amplitudes and can be identified in a good approximation with the mode(s) that is (are) intrinsically energetically unstable and are the origin of the observed structure (with small corrections owing to the presence of frozen secondary modes of the same symmetry). In the case of ambiguity with respect to the possible irrep associated with the primary distortion, a comparison of their respective amplitudes is in most cases sufficient for their identification, and therefore for identifying the mechanism underlying the stabilization of the phase.

\section{An improper ferroelectric: gadolinium molybdate}

A ferroelectric is said to be improper if its polar distortion, responsible for the spontaneous polarization, is a secondary mode (Toledano \& Toledano, 1987). The symmetry of an improper ferroelectric cannot therefore be explained by the presence of a polar distortion. This latter is usually not intrinsically unstable in the parent paraelectric phase and its appearance in the distorted phase is induced by its coupling with a primary unstable non-polar distortion mode. The spontaneous electric polarization in these materials is usually very small compared with that in proper ferroelectrics, as expected from its secondary role in the stabilization of the phase.

$\mathrm{Gd}_{2}\left(\mathrm{MoO}_{4}\right)_{2}$ is a well known improper ferroelectric (Dvorak, 1971; Jeitschko, 1972). Its ferroelectric phase (see Fig. 3) has space group $\mathrm{Pba2}$, with a duplication of the unit cell (transformation: $\mathbf{a}-\mathbf{b}, \mathbf{a}+\mathbf{b}, \mathbf{c} ; 0,1 / 2,0)$ with respect to its parent structure of symmetry $P \overline{4} 2_{1} m$, which is stable above $433 \mathrm{~K}$. The maximum atomic displacement in the displacive distortion is of the order of $0.4 \AA$. Fig. 4 shows a graph of maximal subgroups relating the space groups of both phases, with an indication of the possible irrep distortions of $P \overline{4} 2_{1} m$ compatible with these symmetries. As shown in the graph, we should expect in the Pba2 phase three distortion modes. A primary one yields directly the observed symmetry and corresponds to the (physically irreducible) (see, for example, 


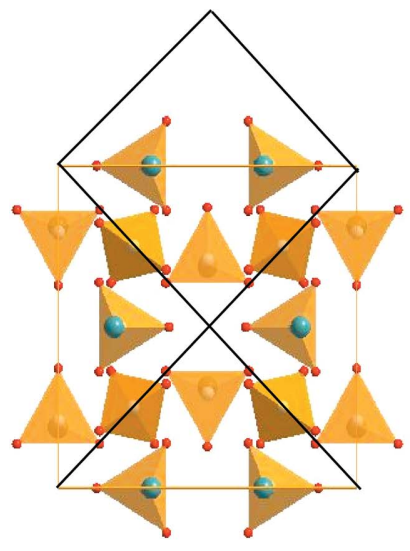

(a)

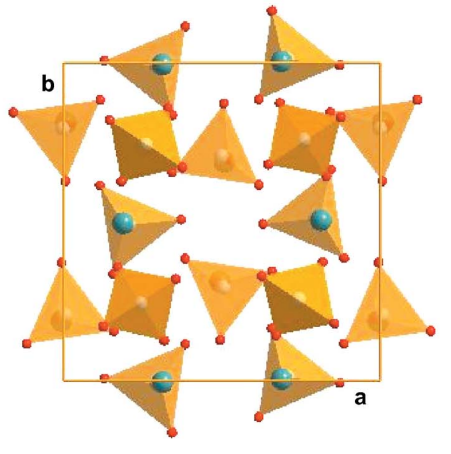

(b)
Figure 3

Structure of $\mathrm{Gd}_{2}\left(\mathrm{MoO}_{4}\right)_{2}$ projected on the $x y$ plane in the parent $P \overline{4} 2_{1} m$ phase (a), and in the distorted $P b a 2$ phase $(b)$. The smaller tetragonal unit cell of the parent phase is indicated in $(a)$.

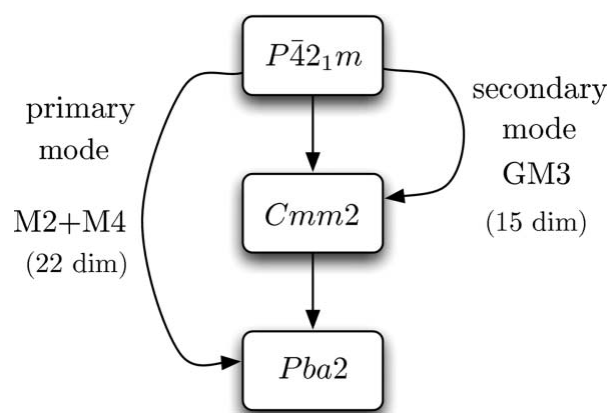

Figure 4

Graph of maximal subgroups relating the space groups of the parent and distorted phases of $\mathrm{Gd}_{2}\left(\mathrm{MoO}_{4}\right)_{2}$. For each subgroup any irrep compatible with it is indicated, together with the dimension of the corresponding distortion subspaces.

Stokes \& Hatch, 1988) irrep M2+M4, associated with the point $\mathrm{M}(1 / 2,1 / 2,0)$ at the border of the Brillouin zone. A second mode at the center of the Brillouin zone with symmetry given by irrep GM3, only breaking the symmetry up to the intermediate subgroup Cmm2, is also symmetry allowed and will also be present as a secondary distortion mode. This second mode is polar and is responsible for the spontaneous polarization of the Pba2 phase. Finally, there can also be a fully symmetric GM1 distortion keeping the parent symmetry. The number of independent symmetry modes corresponding to these symmetries is 22 and 15 for the M2+M4 and GM3 subspaces, respectively, while the subspace of GM1 distortions has 14 dimensions, in accordance with the number of free atomic parameters already present in the parent $P \overline{4} 2_{1} m$ structure. In other words, the determination of the M2+M4, GM3 and GM1 distortions requires 22, 15 and 14 parameters, respectively, so that their total number is 51 , in accordance with the number of free atomic positional parameters in a conventional description of the Pba2 structure (Jeitschko, 1972).

A summary of the mode decomposition of the Pba2 experimental structure reported by Jeitschko (1972) is given in
Table 4

Summary of the mode decomposition (with respect to its $P \overline{4} 2_{1} m$ parent structure) of the $\mathrm{Pba} 2$ structure of $\mathrm{Gd}_{2}\left(\mathrm{MoO}_{4}\right)_{2}$ at $190 \mathrm{~K}$ reported by Jeitschko (1972) (total distortion: $1.63 \AA$ ).

\begin{tabular}{llllll}
\hline K-vector & Irrep & Direction & $\begin{array}{l}\text { Isotropy } \\
\text { subgroup }\end{array}$ & Dimension & $\begin{array}{l}\text { Amplitude } \\
(\AA)\end{array}$ \\
\hline$(0,0,0)$ & GM1 & $(a)$ & $P \overline{4} 2_{1} m$ & 14 & 0.15 \\
$(0,0,0)$ & GM3 & $(a)$ & $C m m 2$ & 15 & 0.07 \\
$(1 / 2,1 / 2,0)$ & M2+M4 & $(a, b)$ & $P b a 2$ & 22 & 1.62 \\
\hline
\end{tabular}

Table 5

Reference structure for $\mathrm{Gd}_{2}\left(\mathrm{MoO}_{4}\right)_{2}$ corresponding to the $P \overline{4} 2_{1} m$ phase in the Pba2 phase setting $(a=10.455281, b=10.455281, c=10.67 \AA)$.

\begin{tabular}{lllll}
\hline & Wyckoff & & & \\
Atom & position & $x$ & 0.50000 & 0.73762 \\
Gd1 & $4 c$ & 0.18744 & 0.81256 & 0.26238 \\
Gd1_2 & $4 c$ & 0.00000 & 0.50000 & 0.35695 \\
Mo1 & $4 c$ & 0.20663 & 0.79337 & 0.64305 \\
Mo1_2 & $4 c$ & 0.00000 & 0.25000 & 0.00000 \\
Mo2 & $4 c$ & 0.25000 & 0.50000 & 0.51950 \\
O1 & $4 c$ & 0.19520 & 0.80480 & 0.48050 \\
O1_2 & $4 c$ & 0.00000 & 0.00000 & 0.31090 \\
O2 & $4 c$ & 0.12890 & 0.87110 & 0.68910 \\
O2_2 & $4 c$ & 0.50000 & 0.13720 & 0.70050 \\
O3 & $4 c$ & 0.13890 & 0.36280 & 0.70050 \\
O3_2 & $4 c$ & 0.36110 & 0.86110 & 0.29950 \\
O3_3 & $4 c$ & 0.63720 & 0.63890 & 0.29950 \\
O3_4 & $4 c$ & 0.86280 & 0.17700 & 0.09550 \\
O4 & $4 c$ & 0.13770 & 0.32300 & 0.09550 \\
O4_2 & $4 c$ & 0.36230 & 0.86230 & 0.90450 \\
O4_3 & $4 c$ & 0.67700 & 0.63770 & 0.90450 \\
O4_4 & $4 c$ & 0.82300 & & \\
\hline
\end{tabular}

Table 4. Again here the primary distortion is dominant, its amplitude being more than one order of magnitude larger than the secondary distortion GM3. In a very good approximation the structure can be described considering only the $\mathrm{M} 2+\mathrm{M} 4$ and the GM1 distortions, i.e. with a significant decrease of $30 \%$ in the number of positional parameters compared with a conventional description.

Tables 5 and 6 complete the symmetry-mode description of the $\mathrm{Pba} 2$ structure of $\mathrm{Gd}_{2}\left(\mathrm{MoO}_{4}\right)_{2}$ in a form following crystallographic conventions. Table 5 lists a $P b a 2$ asymmetric unit, with the atomic positions corresponding to the reference parent phase of higher symmetry (Jeitschko, 1972). For this asymmetric unit, Table 6 lists the atomic displacements in relative coordinates defining the normalized polarization vectors of the GM1, M2+M4 and GM3 distortion modes present in the structure. This information together with the mode amplitudes in Table 4 is sufficient for obtaining, just by adding the three sets of displacements, the atomic coordinates of the asymmetric unit that define the observed $\mathrm{Pba} 2$ structure in a conventional form. This table gives information on the pattern of correlated atomic displacements associated with the modes of different symmetry intervening in the distortion. One can see in Table 6 that the mode GM3 involves mainly atomic displacements on the plane $x y$, while the displacements along $z$, which are the only ones with polar character, are much smaller. In fact, considering the very small amplitude of 
Table 6

Polarization vectors of the GM1, GM3 and M2+M4 distortions present in the $P b a 2$ phase of $\mathrm{Gd}_{2}\left(\mathrm{MoO}_{4}\right)_{2}$.

The atomic displacements for the asymmetric unit listed in Table 5 are listed in relative units with respect to the Pba2 reference unit cell.

\begin{tabular}{|c|c|c|c|c|c|c|c|c|c|}
\hline \multirow[b]{2}{*}{ Atom } & \multicolumn{3}{|l|}{ GM1 } & \multicolumn{3}{|l|}{ GM3 } & \multicolumn{3}{|l|}{$\mathrm{M} 2+\mathrm{M} 4$} \\
\hline & $\delta x$ & $\delta y$ & $\delta z$ & $\delta x$ & $\delta y$ & $\delta z$ & $\delta x$ & $\delta y$ & $\delta z$ \\
\hline Gd1 & 0.0018 & 0.0000 & -0.0022 & 0.0007 & 0.0000 & 0.0004 & 0.0000 & -0.0029 & 0.0000 \\
\hline Gd1_2 & 0.0000 & -0.0018 & 0.0022 & 0.0000 & 0.0007 & 0.0004 & 0.0043 & 0.0000 & 0.0000 \\
\hline Mo1 & -0.0035 & 0.0000 & 0.0026 & -0.0056 & 0.0000 & -0.0069 & 0.0000 & -0.0068 & 0.0000 \\
\hline Mo1_2 & 0.0000 & 0.0035 & -0.0026 & 0.0000 & -0.0056 & -0.0069 & -0.0018 & 0.0000 & 0.0000 \\
\hline Mo2 & 0.0000 & 0.0000 & 0.0000 & 0.0000 & 0.0000 & -0.0057 & -0.0048 & -0.0044 & 0.0000 \\
\hline $\mathrm{O} 1$ & -0.0117 & 0.0000 & -0.0094 & -0.0182 & 0.0000 & 0.0020 & 0.0000 & -0.0073 & 0.0000 \\
\hline O1_2 & 0.0000 & 0.0117 & 0.0094 & 0.0000 & -0.0182 & 0.0020 & 0.0123 & 0.0000 & 0.0000 \\
\hline $\mathrm{O} 2$ & -0.0036 & 0.0000 & -0.0016 & 0.0049 & 0.0000 & 0.0020 & 0.0000 & 0.0043 & 0.0000 \\
\hline $\mathrm{O} 2 \_2$ & 0.0000 & 0.0036 & 0.0016 & 0.0000 & 0.0049 & 0.0020 & 0.0037 & 0.0000 & 0.0000 \\
\hline $\mathrm{O} 3$ & -0.0052 & -0.0073 & -0.0047 & -0.0161 & -0.0010 & 0.0017 & 0.0129 & 0.0122 & -0.0116 \\
\hline O3_2 & 0.0052 & 0.0073 & -0.0047 & 0.0161 & 0.0010 & 0.0017 & 0.0129 & 0.0122 & 0.0116 \\
\hline O3_3 & -0.0073 & 0.0052 & 0.0047 & 0.0010 & -0.0161 & 0.0017 & -0.0129 & 0.0153 & -0.0041 \\
\hline O3_4 & 0.0073 & -0.0052 & 0.0047 & -0.0010 & 0.0161 & 0.0017 & -0.0129 & 0.0153 & 0.0041 \\
\hline O4 & -0.0171 & -0.0063 & 0.0005 & 0.0059 & -0.0066 & 0.0010 & -0.0062 & -0.0029 & -0.0015 \\
\hline O4_2 & 0.0171 & 0.0063 & 0.0005 & -0.0059 & 0.0066 & 0.0010 & -0.0062 & -0.0029 & 0.0015 \\
\hline O4_3 & -0.0063 & 0.0171 & -0.0005 & 0.0066 & 0.0059 & 0.0010 & 0.0038 & 0.0051 & 0.0015 \\
\hline O4_4 & 0.0063 & -0.0171 & -0.0005 & -0.0066 & -0.0059 & 0.0010 & 0.0038 & 0.0051 & -0.0015 \\
\hline
\end{tabular}

this GM3 distortion, its $z$ displacements, except in the case of the Mo atoms, are practically zero within their experimental error. Jeitschko (1972) already pointed out that the estimated value of the spontaneous polarization in this structure considering nominal charges was smaller than its standard deviation.

The fact that the GM3 atomic displacements along $z$ are practically negligible does not mean, however, that the atoms remain static along this direction. They displace indeed along this direction, but following essentially the symmetry pattern corresponding to the mode M2+M4, as shown in Table 6. This means that the total structure has some approximate hidden non-crystallographic atomic correlations which are satisfied within experimental resolution, similar, as it happens, to the orthorhombic phase of $\mathrm{BaTiO}_{3}$ discussed in $\S 3$.

\section{A strongly distorted ferroelastic: leucite}

We consider now the structure of leucite. This mineral, with formula $\mathrm{KAlSi}_{2} \mathrm{O}_{6}$, is tetragonal $\left(I 4_{1} / a\right)$ at room temperature,

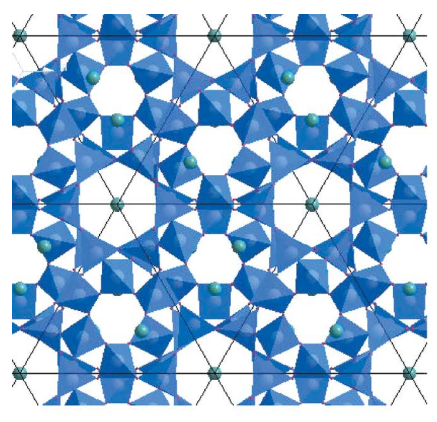

(a)

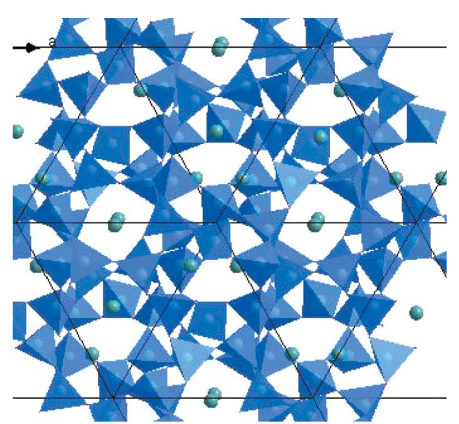

(b)
Figure 5

Structure of leucite $\left(\mathrm{KAlSi}_{2} \mathrm{O}_{6}\right)$ projected along one of its trigonal axis in its cubic phase. (a) High-temperature $I a \overline{3} d$ phase. (b) Room-temperature tetragonal $I 4_{1} / a$ phase (Palmer et al., 1997) but becomes cubic $(\operatorname{Ia} \overline{3} d)$ above approximately $940 \mathrm{~K}$ [see Palmer et al. (1997) and references therein]. An intermediate phase in a very narrow temperature interval with space group $I 4_{1} /$ acd has also been reported (Lange et al., 1986; Palmer et al., 1997). There is a group-subgroup relation between the room-temperature $I 4_{1} / a$ and the high-temperature symmetry $I a \overline{3} d$, but, as can be seen in Fig. 5, the displacive distortion relating both phases is very large. The connected framework of $\mathrm{SiO}_{4}$ and $\mathrm{AlO}_{4}$ tetrahedra suffer a strong rearrangement when passing from the cubic to the tetragonal configuration with a collapse of the cation-stuffed trigonal channels of the cubic phase. In this case the maximum atomic displacement is of the order of $1 \AA$, a value considerably larger than in the examples discussed above. Nevertheless, we will see in the following that despite the large magnitude of the distortion it still can be rationalized in terms of modes. The temperature evolution of the structure includes the variation of two distortion modes with different symmetry, and as a consequence having quite different temperature behavior.

The number of atoms per primitive unit cell is the same in both phases, and therefore only modes at the Brillouin-zone center, i.e. modes keeping the lattice periodicity, are involved in the distortion. It suffices to introduce the experimental structures (Palmer et al., 1997) of the two phases in the program AMPLIMODES, together with the transformation relating the settings of the two space groups $(\mathbf{a}, \mathbf{b}, \mathbf{c} ; 1 / 2,0,0)$, to obtain the amplitudes and the specific features of the irrep distortions present in the tetragonal phase. A scheme of the group-subgroup tree relating the parent and distorted symmetry is shown in Fig. 6. There is a primary distortion (irrep GM4+) which yields the observed symmetry break between the two phases, plus a secondary mode (irrep GM3+) with a higher isotropy subgroup $\left(I 4_{1} /\right.$ acd $)$, and the usual full symmetric distortion mode (irrep GM1+). The 30-dimensional configuration space of the $I 4_{1} / a$ structure (30 independent atomic coordinates define the structure) divides among these 
three distortion subspaces of 16, 10 and 4 dimensions for GM4+, GM3+ and GM1+, respectively. Their amplitudes at room temperature result to be $4.61,1.82$ and $0.41 \AA$. As expected, the primary distortion is significantly larger, although not in such strong proportion as in the other examples discussed above.

Palmer et al. (1997) made a series of high-resolution powder neutron diffraction measurements of leucite as a function of temperature below and above the phase transition around $940 \mathrm{~K}$, and have reported structural models for the material at various temperatures. It is illustrative to analyze these structures in terms of modes and observe the temperature behavior of the three irrep distortions active in the tetragonal phase. Their amplitudes follow a well behaved smooth temperature dependence, shown in Fig. 7(a). Note that apart from the structures determined above room temperature, the study by Palmer et al. (1997) also determined the structure at $4 \mathrm{~K}$. Even the amplitudes corresponding to this isolated point at very low temperature agree with the smooth curves suggested by the high-temperature data. The available points for the amplitude of the GM4+ distortion have been fitted to a continuous function, following the typical law of an order parameter of a discontinuous phase transition (Dove, 1997). What is especially remarkable is that the curve fitted to the GM3+ amplitudes is just the square of the curve used for the GM4+ amplitudes, with only a scale factor having been adjusted. Hence, we are observing a primary component in the structural distortion behaving as the primary order parameter, while a second one, weaker but significant, varies its amplitude as the square of the amplitude of the primary distortion, as expected for a secondary distortion with faintness index 2 (see $\S 4)$. It should be stressed that in general for each individual atomic position the contributions of the two modes superpose, and therefore the simple law underlying the thermal evolution of the structure shown in Fig. 7(a) cannot be directly observed in the thermal changes of single atomic coordinates or atomic distances.

One can also follow the temperature variation of the polarization vectors of the two irrep distortions. The value of their scalar product, with the one corresponding to the structure at $4 \mathrm{~K}$ used as reference, can be used to monitor its

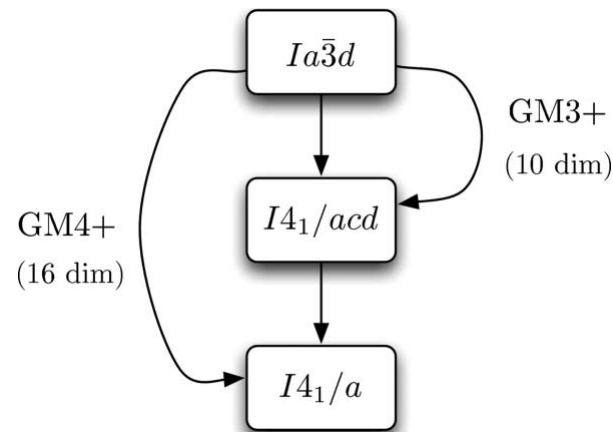

Figure 6

Graph of maximal subgroups relating the space groups of the parent and distorted phases of leucite. For each subgroup the irrep yielding this symmetry is indicated, together with the dimension of the corresponding distortion subspace. change. One can see in Fig. 7(b) that the polarization vectors of both irrep distortions are approximately temperatureinvariant. This means that in each irrep distortion the atoms follow correlated relative displacements which are common to the whole temperature range, the temperature variation being essentially reduced to their global amplitude. This is specially true for the primary mode. For this mode, except for the points closer to the transition, the scalar product maintains values larger than 0.99, and does not decrease in any case below 0.97. The polarization vector of the secondary distortion GM3+ has a more significant variation, but is also quite small, except close to the transition.

The approximate invariance of the polarization vectors of the irrep distortions can be understood in the light of the discussion presented in $\S 4$. If we consider only the lowest anharmonic coupling of the secondary normal modes to the primary one and assume them essentially temperatureindependent as done in Landau theory, the secondary distortion minimizing the free energy will be formed by a linear combination of secondary normal modes that will be kept invariant for changes of the temperature and the amplitude of the primary normal mode. For the primary distortion something similar happens, except that it is expected to have an overwhelming proportion of the primary normal mode. Hence, deviations of the ideal invariance owing to higherorder anharmonic coupling terms are expected to be smaller.

One may argue that the remarkable 'rigidity' of the polarization vectors of the distortion modes is due to the fact that they correspond to rigid unit modes (RUMs) (Giddy et al., 1993; Hammonds et al., 1996) of the tetrahedral framework in the leucite structure. Both irrep distortions can indeed be

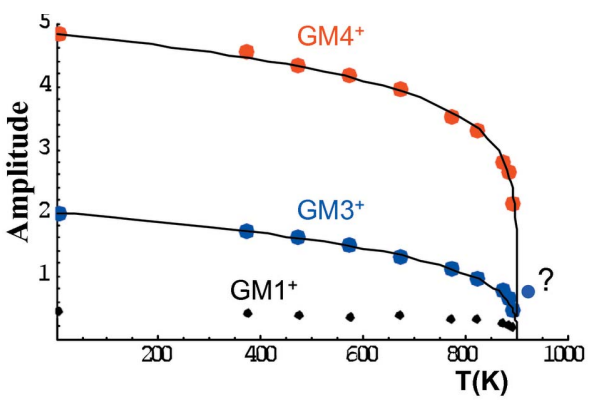

(a)

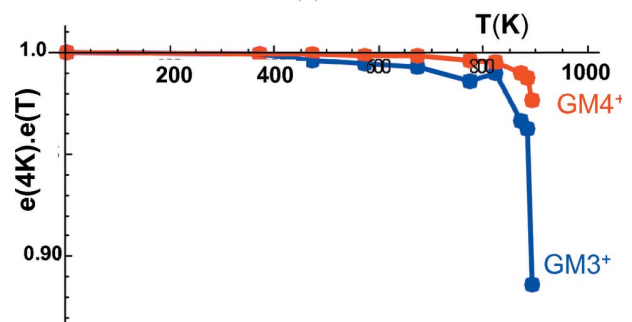

(b)

Figure 7

(a) Temperature dependence of the amplitudes of the primary (GM4+) and secondary (GM3+) distortions in leucite, according to the structures reported by Palmer et al. (1997). (b) Scalar product of the 16- and 10dimensional normalized polarization vectors of the two distortions at each temperature with the corresponding one in the reported structure at $4 \mathrm{~K}$. 
considered RUMs for the framework of $\mathrm{AlO}_{4}$ and $\mathrm{SiO}_{4}$ tetrahedra. But the primary GM4+ distortion includes a significant relative displacement of the $\mathrm{K}$ cations, and its participation in the polarization vector is also invariant. We will see below other examples where connected frameworks of rigid units do not exist, and nevertheless the property of the approximate invariance of the polarization vectors of the irrep distortions is maintained, even between different compounds.

In Fig. 7(b) one can see that the polarization vectors of both the primary and the secondary distortions have their largest variation close to the phase transition. We have also observed this behavior in various other systems. It is not clear if this is a genuine structural feature or an experimental effect coming from the intrinsic difficulty of the measurements in the proximity of a phase transition. It is clear that the experimental uncertainty of the normalized polarization vector increases as the mode amplitude of the mode decreases. The relative decrease of the amplitudes does not seem, however, sufficient to explain this systematic variation of the polarization vectors when the transition is approached. On the other hand, it has been shown in many cases that the primary distortion well below the transition agrees almost $100 \%$ with the primary normal mode calculated by means of $a b$ initio calculations (see $§ 11)$. It can be then hardly understood that this agreement should deteriorate somehow as the system approaches the transition (genuine critical phenomena are irrelevant here as the transitions are either discontinuous or the proximity to the phase transition is not sufficient for these phenomena to appear). The simple model explaining the invariance of the polarization vectors of the irrep distortions is in principle expected to be more appropriate for smaller distortions. Therefore, we speculate that the significant fluctuations of the distortion polarization vectors close to the transition points are an indication of a poorer determination of the structures under these conditions.

It is remarkable that the intermediate phase of symmetry $I 4_{1} /$ acd, which has been proposed for a small temperature interval above $900 \mathrm{~K}$, just before reaching the parent cubic configuration, would correspond to a primary distortion of symmetry GM3+. A distortion of this symmetry is also present at room temperature, but only as a secondary induced distortion, as discussed above and shown in Fig. 7(a). It is then rather peculiar to have a distortion of this symmetry acting as a primary (unstable) mode at higher temperatures. The mode decomposition of the $I 4_{1} /$ acd structural model at $T=923 \mathrm{~K}$ of Palmer et al. (1997) yields an amplitude for this distortion of $0.86 \AA$, also shown in Fig. $7(a)$. This value is clearly at odds with the temperature behavior of the amplitude of the GM3+ distortion in the $I 4_{1} / a$ phase, which shows a fast but smooth tendency to zero at a temperature around $900 \mathrm{~K}$. The polarization vector of the GM3+ distortion mode in the $I 4_{1} /$ acd structural model is in fact very different from the one corresponding to the $I 4_{1} / a$ phase, indicating that it is a quite different type of distortion despite having the same symmetry.

A primary mode responsible for a distorted phase is expected to be intrinsically unstable, with temperature acting as stabilizing factor, so that in general its disappearance at lower temperatures is usually caused by its incompatibility with new stronger instabilities of different symmetry. This is not the case here, since the phase $I 4_{1} / a$ produced by the primary GM4+ distortion mode is compatible with any GM3+ distortion. Of course, there can be exceptional cases of reentrant transitions, but this intermediate $I 4_{1} /$ acd phase would be even a more exceptional case, since the GM3+ distortion present would disappear when the system enters the $I 4_{1} / a$ phase, although it is fully compatible with this symmetry. We can therefore conclude that most probably the symmetry and structural model proposed for this intermediate hightemperature phase is not correct.

\section{Domains and equivalent structures}

It is well known that, for a given distorted structure with symmetry $\mathcal{H}<\mathcal{G}$, there is a series of physically equivalent structures which are crystallographically distinguishable when referred to the common parent structure, i.e. the so-called domains, variants or twin-related structures (Janovec \& Privratska, 2003).

If the left-coset decomposition of $\mathcal{G}$ with respect to $\mathcal{H}$ is given by

$$
\mathcal{G}=\mathcal{H}+g_{2} \mathcal{H}+\ldots+g_{n} \mathcal{H}
$$

with $n$ being the index of the subgroup $\mathcal{H}$, the application of the $\mathcal{G}$ operations chosen as coset representatives $g_{2}, \ldots, g_{n}$ on the distorted structure $\mathcal{H}$ produces $n-1$ structures equivalent to the original one, which together with this latter correspond to the expected $n$ distinct domain configurations. The spacegroup symmetries of these $n$ equivalent structures are given by the space groups $\mathcal{H}_{i}$, of the same type as $\mathcal{H}$ and given by $g_{i} \mathcal{H} g_{i}^{-1}$. From the viewpoint of the configuration energy map discussed in $\$ 4$, it means that there are $n$ equivalent energy minima around the saddle point associated with the parent phase, giving place to the multistability or degeneracy of the distorted phase, with the possibility of switching processes through external fields between the different equivalent energy minima.

The mode decomposition as formalized in the previous sections is restricted to a specific orientation (and origin shift) between the subgroup $\mathcal{H}$ and $\mathcal{G}$, given by the transformation matrix $(P, p)$ relating the two space groups. This means that among the possible equivalent orientations and origin relations between the low- and high-symmetry structures a choice must be made, and the specific expressions for the polarization vectors of the irrep distortions describing the structure will depend on it. The amplitudes of the irrep distortions are, however, independent of the choice of subgroup $\mathcal{H}$, among the equivalent ones.

In many cases some coset representatives $g_{i}$ are such that

$$
g_{i} \mathcal{H} g_{i}^{-1}=\mathcal{H} \text {. }
$$

This means that the symmetry of the corresponding domainequivalent configuration is described by the same space group $\mathcal{H}$, and the use of a specific transformation $(P, p)$ between the parent space group and the space group of the distorted 
structure does not fully specify the domain choice. Domainrelated structures with the same space group $\mathcal{H}$ [the same transformation $(P, p)]$ are distinguished because their mode decomposition yields irrep distortions with the same polarization vectors but some of them having amplitudes with opposite sign, or equivalently the polarization vectors of some irrep distortions have a global change of sign, if by definition the irrep distortion amplitudes are chosen positive.

If a distorted structure is described by a set of irrep distortions with amplitudes $\left\{A_{\tau 1}, A_{\tau 2}, \ldots, A_{\tau s}\right\}$, and $g_{i}$ is a coset representative in (12) such that (13) is satisfied, then an equivalent domain-related structure is obtained considering the same polarization vectors for the irrep distortions and transforming the amplitudes through the action of the spacegroup operation reduced to a +1 or -1 factor, i.e. $\left\{\tau_{1}\left(g_{i}\right) A_{\tau 1}, \tau_{2}\left(g_{i}\right) A_{\tau 2}, \ldots, \tau_{s}\left(g_{i}\right) A_{\tau s}\right\}$, where $\tau_{j}\left(g_{i}\right)$ are +1 or -1 according to the transformation properties of each amplitude given by the corresponding irrep. ${ }^{3}$ The values of the factors $\tau_{j}\left(g_{i}\right)$ are in general correlated. Those of the secondary modes can be directly derived from those of the primary modes through the relation (9) connecting the amplitudes of the secondary distortions to the primary ones (this equation considers the lowest coupling terms among primary and secondary distortions, but the resulting sign correlation is a symmetry property that is maintained at any level of approximation). If the distorted structure contains a single primary distortion, the mode decomposition of the domainrelated configuration will yield a primary distortion with opposite sign, while secondary distortions will change sign or not depending on their faintness index (see §4) being odd or even. If, on the contrary, there are several primary distortions (with different irreps) in the structure, more than two domainequivalent configurations for the same fixed subgroup $\mathcal{H}$ exist [i.e. more than one coset representative $g_{i}$ fulfills (13)], and the different domains will be distinguished by independent uncorrelated changes of sign of the different primary distortions, while secondary distortions will have their signs forced by those of the primary ones according to (9), or similar generalized equations.

The subset of equivalent domain configurations corresponding to a fixed $\mathcal{H}$ and obtained by the allowed changes of sign of the primary distortions (and correlated ones of the secondary ones) correspond to some of the different equivalent crystallographic descriptions of the same structure, obtained by means of transformations belonging to the Euclidean normalizer of the group $\mathcal{H}$ extended to the possible specialized metric of the lattice of $\mathcal{H}$, when its strain with respect to the lattice of the supergroup $\mathcal{G}$ is neglected (Koch \& Fischer, 2006).

We can consider two of the previously discussed structures as simple examples of the above considerations. In the case of the $A m m 2$ phase of $\mathrm{BaTiO}_{3}$ there are 12 equivalent distorted structures of this symmetry with respect to the $P m \overline{3} m$ perfect

\footnotetext{
${ }^{3}$ In some cases the action of the lost symmetry operation $g_{i}$ on a given irrep distortion, even when fulfilling (13), cannot be reduced to a factor +1 and -1 , and the polarization vectors are distinguished by more complex rotational relations.
}

perovskite. They correspond to the six different distinct subgroups $A m m 2$ of $P m \overline{3} m$ belonging to the same conjugacy equivalence class, and associated with six different orientations of the rotational operations of the group Amm2 with respect to the $P m \overline{3} m$ setting. Once one of these subgroups has been chosen by means of the transformation $\mathbf{c}, \mathbf{a}-\mathbf{b}, \mathbf{a}+\mathbf{b}$; $0,0,0$, the distorted structure can have two equivalent configurations related by the lost inversion operation, which can be taken as the coset representative fulfilling (13). The inversion operation changes the sign of both the primary polar distortion of symmetry GM4- and the secondary one GM5-. Hence the two equivalent structures can be described by opposite amplitudes $\left(A_{\mathrm{GM}_{4}}, A_{\mathrm{GM} 5-}\right)$ and $\left(-A_{\mathrm{GM}_{4}-},-A_{\mathrm{GM} 5_{-}}\right)$, both keeping unchanged their polarization vectors. As in a mode decomposition the amplitudes are usually chosen positive by definition, the change of sign will be reflected in the polarization vectors considered, which would be opposite in both configurations. The correlated switch of the sign of both distortions is consistent with the faintness index of the GM5- distortion, which is 3, as can be easily checked using the program INVARIANTS of the package ISOTROPY (Stokes \& Hatch, 2002). Note that this means that, although an external electric field only couples linearly with the polar distortion GM4-, it can switch not only this primary polar mode but also the secondary non-polar one GM5- through the anharmonic coupling between the two distortions.

In the second example, gadolinium molybdate, the index of the subgroup is 4 , and we can choose as coset representatives the following operations: $\left(\begin{array}{lll}1 \mid 0 & 0 & 0\end{array}\right),\left(\begin{array}{l}4 \\ z\end{array} \mid 000\right),(1 \mid 100)$, $\left(\begin{array}{lll}4 & z\end{array} \mid \begin{array}{ll}1 & 0\end{array}\right)$. The rotoinversion operation $\overline{4} z$ changes the orientation of the Pba2 space group, and corresponds to another choice of the transformation matrix $(P, p)$. On the other hand, the lost translation (1/1 000$)$ transforms the distorted structure into its so-called antiphase domain. This translational operation only changes the sign of the primary mode, while keeping the signs of the two secondary distortions GM4 and GM1. Thus, once the transformation $(P, p)$ relating both structures is fixed, and maintaining the same polarization vectors, the two alternative domains for this compound correspond to the distortion amplitudes $\left(A_{\mathrm{M} 2+\mathrm{M} 4}, A_{\mathrm{GM} 4}, A_{\mathrm{GM} 1}\right)$ and $\left(-A_{\mathrm{M} 2+\mathrm{M} 4}, A_{\mathrm{GM} 4}, A_{\mathrm{GM} 1}\right)$. These correspond to two equivalent structures related by the operation of the Euclidean normalizer of $P b a 2,(1 \mid 1 / 21 / 2$ 0), i.e. a mere origin shift. Hence, contra-intuitively, the sense of the spontaneous polarization is fixed and unique in the two domains that have the same orientation for the Pba2 space group, despite the system being an improper ferroelectric. The polarization can indeed be switched by means of an electric field, but this corresponds to the transformation into the domain related by the coset representative $(\overline{4} z \mid 000)$, and therefore also implies a transformation of the polarization vector corresponding to the primary distortion $\mathrm{M} 2+\mathrm{M} 4$ according to the action of the point-group operation $\overline{4} z$, which is not reducible to a mere change of sign, but produces a different relative orientation of the $\mathrm{Pba2}$ space group with respect to the tetragonal parent structure. This means that the reversal of the spontaneous 


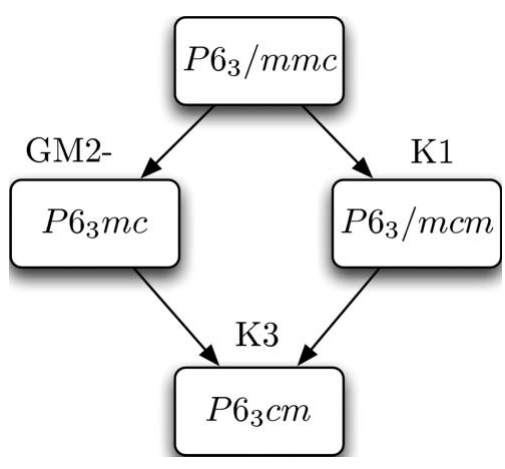

Figure 8

Graph of maximal subgroups relating the space groups of the parent and distorted phases of $\mathrm{KNiCl}_{3}$. For each subgroup any irrep yielding this symmetry is indicated.

polarization along the pseudo-tetragonal $z$ axis through the action of an electric field will be accompanied by the transformation through the operation $\overline{4} z$ of the non-polar M2+M4 atomic displacements described in Table 6, which are mostly on the plane $x y$.

Below we will show further examples where two primary distortion modes are active and the set of possible domains is more varied.

\section{Hexagonal perovskites $A B X_{3}$}

There are a considerable number of $A B X_{3}$ compounds which crystallize in the so-called hexagonal perovskite (2L) structure or in slightly distorted modifications of it. A representative of the parent hexagonal perovskite structure, with $\mathrm{Pb}_{3} / \mathrm{mmc}$ symmetry, underlying these structures is $\mathrm{CsNiCl}_{3}$ (Minkiewicz et al., 1970). For smaller $A$ cations the structure is usually distorted at room or lower temperatures, and polar configurations are rather common, producing ferroelectric phases (Mitsui et al., 2000; Hendrikse \& Maaskant, 1997; Yamanaka et al., 2002). If the $B$ cations are magnetic, magnetically ordered phases also exist at low temperatures, and multiferroic properties combining ferroelectricity and magnetic ordering are possible (Morishita et al., 2001). We will see here that it is very illustrative and illuminating to analyze and compare the structures of this family, performing a systematic mode decomposition with respect to the ideal hexagonal perovskite configuration.

We first consider a representative of the family, namely the compound $\mathrm{KNiCl}_{3}$ (Visser et al., 1980). In the parent $P 6_{3} / m m c$ phase $\mathrm{Ni}, \mathrm{K}$ and $\mathrm{O}$ occupy positions $2 a, 2 d$ and $6 h$. It has a ferroelectric phase at room temperature with space group $\mathrm{Pb}_{3} \mathrm{~cm}$ with a triplicated unit cell $(\mathbf{a}+2 \mathbf{b},-2 \mathbf{a}-\mathbf{b}, \mathbf{c} ; 0,0,0)$. A mode analysis of this phase was done 'by hand' by Mañes $e t$ al. (1982). We present here its mode decomposition as obtained with AMPLIMODES, using the parameterization discussed above. The graph of maximal subgroups and irreps relating the symmetry of the ferroelectric phase with that of

\footnotetext{
${ }^{4}$ In Mañes et al. (1982) the label used was K4. Here we maintain the labels provided by AMPLIMODES in accordance with the convention of ISOTROPY.
}

Table 7

Summary of the mode decomposition with respect to its $P 6_{3} / m m c$ parent structure of the $P 6_{3} \mathrm{~cm}$ structure of $\mathrm{KNiCl}_{3}$ (Visser et al., 1980).

As reference a symmetrized idealized $P 6_{3} / m m c$ structure has been used (see Table 8). (Total distortion: $1.72 \AA$.)

\begin{tabular}{llllll}
\hline K-vector & Irrep & Direction & $\begin{array}{l}\text { Isotropy } \\
\text { subgroup }\end{array}$ & Dimension & $\begin{array}{l}\text { Amplitude } \\
(\AA)\end{array}$ \\
\hline$(0,0,0)$ & GM1+ & $(a)$ & $P 6_{3} / m m c$ & 1 & 0.02 \\
$(0,0,0)$ & GM2- & $(a)$ & $P 6_{3} m c$ & 3 & 0.22 \\
$(1 / 3,1 / 3,0)$ & K1 & $(a, 0)$ & $P 6_{3} / m c m$ & 3 & 0.07 \\
$(1 / 3,1 / 3,0)$ & K3 & $(a, 0)$ & $P 6_{3} c m$ & 2 & 1.70 \\
\hline
\end{tabular}

\section{Table 8}

Reference structure for $\mathrm{KNiCl}_{3}$ corresponding to its parent hexagonal $P 6_{3} / m m c$ phase in the $P 6_{3} \mathrm{~cm}$ setting of its distorted structure $(a=11.795$, $b=11.795, c=5.926 \AA$ ).

\begin{tabular}{lllll}
\hline Atom & $\begin{array}{l}\text { Wyckoff } \\
\text { position }\end{array}$ & $x$ & $y$ & $z$ \\
\hline Ni1 & $2 a$ & 0.000000 & 0.000000 & 0.000000 \\
Ni1_2 & $4 b$ & 0.666667 & 0.333333 & 0.000000 \\
K1 & $6 c$ & 0.333334 & 0.000000 & 0.750000 \\
C1 & $6 c$ & 0.160000 & 0.000000 & 0.250000 \\
C1_2 & $12 d$ & 0.826667 & 0.333333 & 0.250000 \\
\hline
\end{tabular}

the parent phase is shown in Fig. 8. One can see that there is a primary active irrep with wavevector $(1 / 3,1 / 3,0)$ and label $\mathrm{K} 3^{4}$ and two secondary active irreps associated with two intermediate subgroups. The $\mathrm{K} 1$ distortion also corresponds to a wavevector $(1 / 3,1 / 3,0)$, so that it produces the same cell multiplication, but maintains the point group of the parent phase, while the second distortion, GM2- at the Brillouinzone center keeps the parent lattice and is the polar mode responsible for the spontaneous polarization in the distorted phase.

From Fig. 8 it can be deduced that the symmetry break from the parent phase to the room-temperature structure could take place by means of two quite different mechanisms. The most obvious one would be a single phase transition with K3 acting as active primary irrep, and distortions GM2- and K1 appearing as secondary effects. But one could imagine a more complex symmetry-breaking mechanism, with GM2 - and K1 being primary unstable modes, and producing in general two phase transitions, with an intermediate phase of symmetry $P 6_{3} \mathrm{~cm}$ or $P 6_{3} / \mathrm{mmc}$, depending on which of the two distortions first becomes zero as temperature is increased. This alternative mechanism has in fact been considered as a possibility in the case of $\mathrm{YMnO}_{3}$, which has a similar symmetry relation with its parent space group, although both parent and distorted structures are quite different (Nénert et al., 2007; Lonkai et al., 2004).

In the case of $\mathrm{KNiCl}_{3}$, as in $\mathrm{YMnO}_{3}$ (Fennie \& Rabe, 2005; Orobengoa et al., 2009), however, the mode decomposition in terms of the three symmetry-breaking components K3, K1 and GM2 - of the structure of $\mathrm{KNiCl}_{3}$ leaves little room for speculation. Their respective amplitudes (in $\AA$ ) are listed in Table 7. The much larger amplitude of the $\mathrm{K} 3$ antiferrodistortive distortion is a clear indication that it can be identified with the primary order parameter of this phase, and 


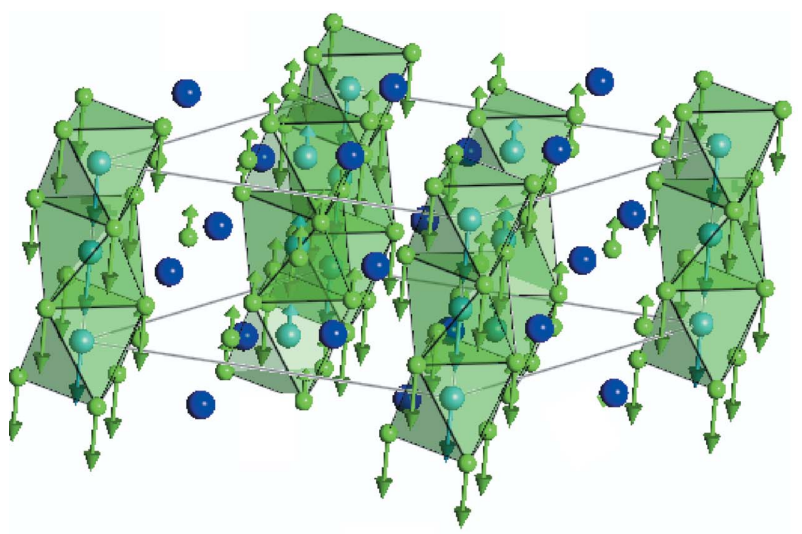

(a)

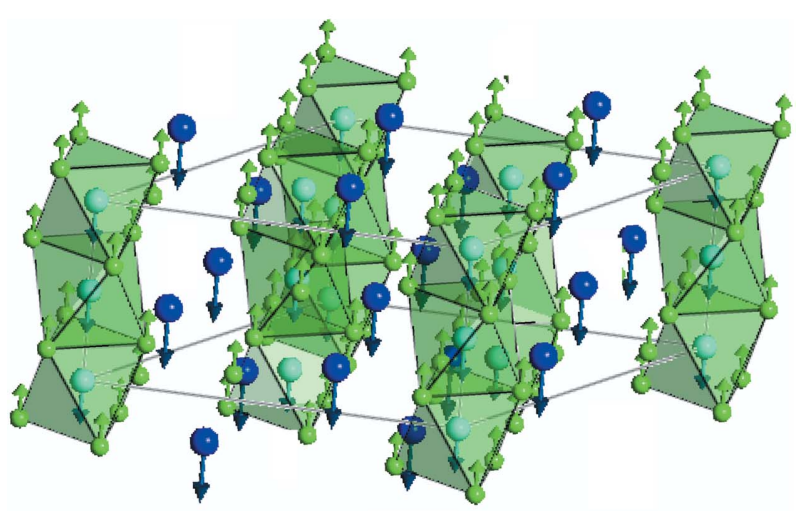

(b)

Figure 9

Polarization vector of the distortions corresponding to the irreps K3 (a) and GM2- $(b)$ in $\mathrm{KNiCl}_{3}$. The scale of the displacement vectors has been enlarged. [Figure created using FullProf Studio (Rodríguez-Carvajal, 1993).]

it can be further inferred that $\mathrm{K} 1$ and GM2- are induced secondary effects. The material is then a ferroelectric of improper character. Figs. 9 and 10 illustrate the polarization vectors of the three types of distortions intervening with so different amplitudes in the total observed distortion. The primary $\mathrm{K} 3$ distortion involves displacements of the columns of $\mathrm{NiCl}_{6}$ octahedra along the $z$ axis, with the two internal columns displacing in opposite direction to the one at the origin. The magnitude of the displacement of the column at the origin doubles that of the internal columns. This noncrystallographic correlation introduced by the $\mathrm{K} 3$ symmetry is patent in the polarization vector listed in Table 9. The Ni1 and Ni1_2 atoms within the asymmetric unit of the distorted structure move in opposite directions along $z$, with a $1 / 2$ relation between their displacements. The same relation exists among the displacements of the two $\mathrm{Cl}$ sites. But Table 9 shows an additional correlation between the displacements of the $\mathrm{Cl}$ and $\mathrm{Ni}$ sites, namely their displacements within each column of $\mathrm{NiCl}_{6}$ octahedra are practically equal within experimental resolution, so that the distortion mode involves global displacements of the $\mathrm{NiCl}_{3}$ columns as rigid units. This correlation of the primary $\mathrm{K} 3$ distortion is not forced either by symmetry or by a strong rigidity of the Ni positions within the octahedra. In fact, in the observed structure the $\mathrm{Ni}$ atoms
Table 9

Polarization vectors of the $\mathrm{K} 3, \mathrm{~K} 1$ and GM2- distortions present in the $\mathrm{Pb}_{3} \mathrm{~cm}$ structure of $\mathrm{KNiCl}_{3}$.

The asymmetric unit is that of Table 8 . Displacements are given in relative units with respect to the reference unit cell (Table 8). Polarization vectors are normalized to $1 \AA$.

\begin{tabular}{|c|c|c|c|c|}
\hline \multirow[b]{2}{*}{ Atom } & \multirow{2}{*}{$\frac{\mathrm{K} 3}{\delta \mathrm{z}}$} & \multicolumn{2}{|l|}{$\mathrm{K} 1$} & \multirow{2}{*}{$\frac{\mathrm{GM} 2-}{\delta \mathrm{z}}$} \\
\hline & & $\delta \mathrm{x}$ & $\delta \mathrm{y}$ & \\
\hline Ni1 & -0.0482 & 0.0000 & 0.0000 & -0.0311 \\
\hline Ni1_2 & 0.0241 & 0.0000 & 0.0000 & -0.0311 \\
\hline K1 & 0.0000 & 0.0288 & 0.0000 & -0.0437 \\
\hline Cl1 & -0.0489 & -0.0078 & 0.0000 & 0.0249 \\
\hline Cl1_2 & 0.0244 & -0.0029 & -0.0137 & 0.0249 \\
\hline
\end{tabular}

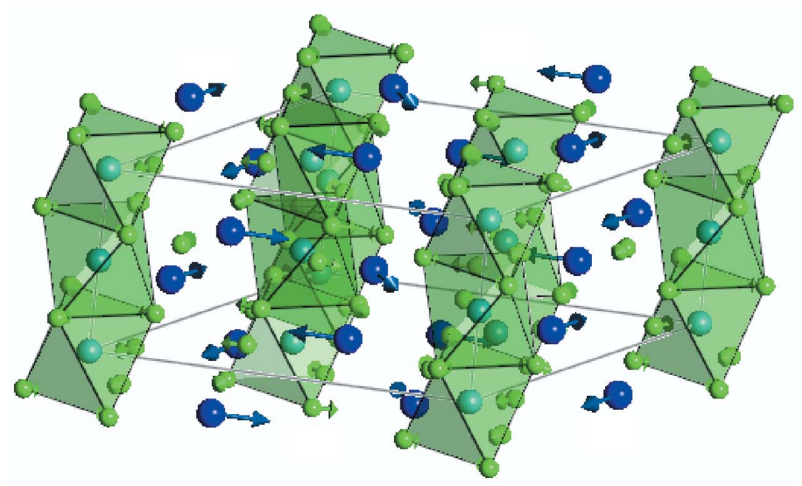

(a)

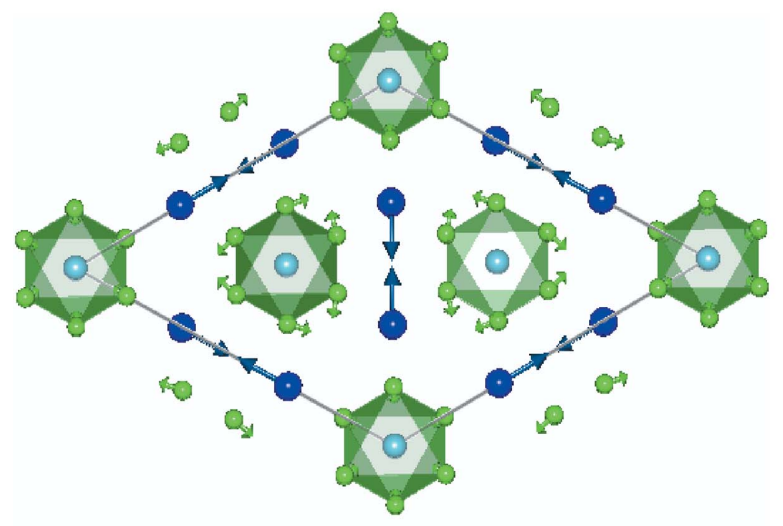

(b)

Figure 10

Polarization vector of the distortion corresponding to the irrep K1 in $\mathrm{KNiCl}_{3}$, in a perspective view $(a)$ and projected on the plane $x y(b)$. The scale of the displacement vectors has been enlarged. [Figure created using FullProf Studio (Rodríguez-Carvajal, 1993).]

clearly displace relatively to their surrounding $\mathrm{Cl}_{6}$ octahedra and approach along the $z$ axis one of the two $\mathrm{Cl}_{3}$ triangles forming the octahedron. But these $\mathrm{Ni}$ displacements are not part of the $\mathrm{K} 3$ distortion; they follow a pattern according to the GM2- symmetry, as shown in Fig. 9(b).

In the GM2- distortion all $\mathrm{Ni}$ atoms move in phase with the same amplitude, while the $\mathrm{Cl}$ atoms displace also in phase with similar amplitude but in opposite sense. The GM2- distortion is completed with the displacements of the $\mathrm{K}$ atoms outside the octahedral columns, which move in the same direction as the $\mathrm{Ni}$ atoms. Hence we have a polar distortion with cations 
Table 10

Comparison of the mode decomposition of the $\mathrm{Pb}_{3} \mathrm{~cm}$ phase of different $A B X_{3}$ compounds.

The third column indicates the dimension of each distortion subspace. For each compound the first row shows the amplitude of the irrep distortion, while the second indicates the value of the scalar product of its polarization vector with that of the corresponding distortion in $\mathrm{KNiCl}_{3}$. For $\mathrm{GM}_{2}-$, the scalar product with that of $\mathrm{TlCoCl}_{3}$ is also indicated in parentheses. The structural models have been taken from Visser et al. (1980) $\left(\mathrm{KNiCl}_{3}\right)$, Jouini et al. (1982) $\left(\mathrm{TlFeBr}_{3}\right), \mathrm{Fink} \&$ Selfert (1982) $\left(\mathrm{RbMnBr}_{3}\right)$, Cussen \& Battle (2000) $\left(\mathrm{BaMnO}_{3}\right.$ at $\left.80 \mathrm{~K}\right)$ and Nishiwaki et al. (2006) $\left(\mathrm{TlCoCl}_{3}\right)$.

\begin{tabular}{|c|c|c|c|c|c|c|c|c|}
\hline & $\begin{array}{l}\text { Isotropy } \\
\text { subgroup }\end{array}$ & Dimension & & $\mathrm{KNiCl}_{3}$ & $\mathrm{TlFeBr}_{3}$ & $\mathrm{RbMnBr}_{3}$ & $\mathrm{BaMnO}_{3}$ & $\mathrm{TlCoCl}_{3}$ \\
\hline \multirow[t]{2}{*}{ GM2- } & $P 6_{3} m c$ & 3 & Amplitude & 0.21 & 0.36 & 0.39 & 0.14 & 0.16 \\
\hline & \multirow{2}{*}{$\mathrm{PG}_{3} / \mathrm{mcm}$} & & Product & $1(0.70)$ & $0.98(0.53)$ & $0.77(0.994)$ & $-0.74(-0.997)$ & $0.70(1)$ \\
\hline & & & Product & 1 & 0.70 & 0.67 & -0.55 & -0.89 \\
\hline $\mathrm{K} 3$ & \multirow{2}{*}{$P 6_{3} \mathrm{~cm}$} & 2 & Amplitude & 1.70 & 1.15 & 1.72 & 0.42 & 1.02 \\
\hline & & & Product & 1 & -0.9997 & 1.0000 & -0.9999 & 0.9999 \\
\hline
\end{tabular}

and anions moving in opposite senses, and therefore susceptible to producing a significant polarization, as observed experimentally (Mitsui et al., 2000). The mode decomposition also shows that the displacement off-center of the $\mathrm{Ni}$ atoms within the $\mathrm{NiCl}_{6}$ octahedra is not part of the fundamental distortion (normal mode) that is unstable. This means that most probably these off-center shifts of the Ni atoms are not intrinsically favorable in contrast with the pure K3 distortion.

It should be stressed that by definition a distortion mode with a non-zero wavevector cannot induce any polarization, and only polar modes at the Brillouin-zone center, such as GM2-, can be considered at the origin of any spontaneous polarization and can linearly couple with an external electric field. This is rather often overlooked in the literature, and in the present compound has led to speculations about possible ferrielectric properties (Machida et al., 1994). From Fig. 9 one can clearly see that the polar displacements of the GM2distortion are fully homogeneous. Ferrielectricity cannot therefore be supported by the experimental structure, and the confusion probably originates in the erroneous consideration of the dominating $\mathrm{K} 3$ distortion pattern (with opposite displacements of the octahedral columns) as the source of the spontaneous polarization.

The secondary distortion $\mathrm{K} 1$, although quite small, seems to be significant within experimental resolution. As shown in Fig. 10 and Table 9, this distortion concerns displacements of the $\mathrm{Cl}$ and $\mathrm{K}$ atoms on the $x y$ plane. The $\mathrm{Cl}$ atoms in consecutive $\mathrm{Cl}_{3}$ triangles along the octahedral $\mathrm{NiCl}_{3}$ columns rotate in opposite senses around the $z$ axis, while the displacements of the $\mathrm{K}$ atoms between the columns move in a sense consistent with the expected steric hindrances caused by the chlorine displacements.

The faintness index (see $\$ 4$ ) of the secondary distortion K1 is 2 , while that of the polar mode GM2 - is $3 .{ }^{5}$ Hence, the signs of the amplitudes of the secondary distortions are bound to those of the primary mode, according to the proportionality laws,

$$
\begin{array}{r}
A_{\mathrm{K} 1} \propto A_{\mathrm{K} 3}^{2}, \\
A_{\mathrm{GM} 2-} \propto A_{\mathrm{K} 3}^{3} .
\end{array}
$$

\footnotetext{
${ }^{5}$ This can be derived using the program INVARIANTS from the package ISOTROPY (Hatch \& Stokes, 1985).
}

This means that an equivalent structure or domain (see §7) will be given by the following changes in the signs of the amplitudes: $\left(-A_{\mathrm{K} 3}, A_{\mathrm{K} 1},-A_{\mathrm{GM}_{2}-}\right)$. These domain sign relations are very important for comparing the mode decomposition of similar or closely related structures coming from different sources or experiments. Table 10 compares the mode decomposition of $\mathrm{KNiCl}_{3}$ with those of other $A B X_{3} P_{3} \mathrm{~cm}$ structures considered isomorphic, namely $\mathrm{TlFeBr}_{3}, \mathrm{RbMnBr}_{3}, \mathrm{BaMnO}_{3}$ and $\mathrm{TlCoCl}_{3}{ }^{6}$ The amplitudes of the three irrep distortions for each compound are listed, and their polarization vectors are compared through their scalar product with that of $\mathrm{KNiCl}_{3}$. One can see that in the five compounds the distortion K3 is predominant and has within experimental resolution the same bidimensional polarization vector, which means that in all cases the octahedral $B X_{3}$ columns displace as rigid bodies, including the $B$ cations inside the $B X_{6}$ octahedra. The polar distortion GM2- has, however, clear differences. Although the GM2- distortion in $\mathrm{TlFeBr}_{3}$ is essentially the same as in $\mathrm{KNiCl}_{3}$, the positive sign of their scalar product is inconsistent with the change of sign observed in the primary distortion $\mathrm{K} 3$. This means that in $\mathrm{TlFeBr}_{3}$ the polar displacements of the $A$ and $B$ cations and $X_{3}$ columns along $z$ displayed in Fig. $9(b)$ have opposite senses with respect to the sense taken by the primary K3 distortion. In other words, an equivalent K3 distortion in both compounds would produce a spontaneous polarization in opposite directions. This structure must then be taken with caution. It may happen that indeed the anharmonic couplings between similar K3 and GM2- distortions in the two compounds have opposite signs, but another reason could be that one of the two models corresponds to a local false minimum in the refinement process. Indeed, false minima have been reported in the least-square minimization of distorted structures corresponding to changes of sign of some of the irrep distortion modes present in the actual structure (Rae $e t$ al., 1992).

The sign of the scalar products of the polarization vectors of the GM2- distortions in the other three compounds is consistent when compared with the scalar product of the K3

\footnotetext{
${ }^{6}$ The Inorganic Crystal Structure Database (Bergerhoff \& Brown, 1987) contains a few additional cases with vanadium as $B$ cation, and $\mathrm{RbTiI}_{3}$ from Zandbergen (1981), but unfortunately in these models the $z$ coordinate of two atoms (instead of one) were a priori fixed in the refined model, and therefore the structural models cannot be considered realistic.
} 
mode. However, the three distortions differ significantly from that of $\mathrm{KNiCl}_{3}$, as its scalar product is only of the order of $0.7-$ 0.8 . This difference can be further assessed by comparing the atomic displacements associated with the distortion mode in each case. Table 11 shows the polarization vector of the GM2- distortion mode of $\mathrm{TlCoCl}_{3}$. One can see that in contrast with $\mathrm{KNiCl}_{3}$ the $B$ cations displace along $z$ in the same sense as the chlorine atoms, and with almost the same amplitude, so that in this case the octahedral $\mathrm{BCl}_{3}$ columns essentially move as rigid units in the polar distortion. The very small off-center shift of the $B$ cations within the octahedra is close to the experimental error and in fact in the opposite direction to that observed in $\mathrm{KNiCl}_{3}$. We can then expect the spontaneous polarization to be much weaker than in $\mathrm{KNiCl}_{3}$.

The GM2 - distortion in $\mathrm{BaMnO}_{3}$ and $\mathrm{RbMnBr}_{3}$ is very similar to the one of $\mathrm{TlCoCl}_{3}$. This can be seen in Table 10, where the alternative scalar product with respect to the polarization vector of this distortion in $\mathrm{TlCoCl}_{3}$ is also shown. One can observe therefore that, despite the similarities among the structures, a clear difference exists between the $\mathrm{Ni}$ and the $\mathrm{Mn} / \mathrm{Co}$ compounds with respect to the form of the secondary polar distortion.

The very weak marginal K1 distortion has much larger variations between the compounds. As its amplitude is much smaller than the other two irrep distortions, its polarization wavevector is expected to have a larger error. In some of the compounds its amplitude is so small, as in $\mathrm{TlCoCl}_{3}$, that it could be considered negligible. For this compound, note that although the polarization vector of the $\mathrm{K} 1$ distortion is rather close to that of $\mathrm{KNiCl}_{3}$ (see Tables 9 and 11), with the displacement of $A$ cations and $\mathrm{Cl}$ anions being correlated in a similar form (see Fig. 9), its sign is inconsistent with that in $\mathrm{KNiCl}_{3}$. In some of the other compounds the polarization vector is quite different and the sense of the displacements of the $A$ cations relative to those of the $X$ anions changes.

One can then summarize that the primary distortion mode is quite robust and transportable from one material to another, while the secondary distortions can vary considerably. In some cases this could be due to false local minima in the refinement process, while in other cases it can happen because of genuine changes in the scheme of harmonic and anharmonic couplings among the atomic displacements between different compounds. In any case, secondary modes have much smaller amplitudes and are bound to be more poorly determined. A look at the structure decomposed into modes can be very useful both to avoid the traps of false refinement minima, and to compare structures, where the differences appear mostly in changes of the secondary distortions, which although quite weak can be fundamental for the macroscopic properties of the material.

Some of the studies on these compounds have indicated that the alternative symmetry $P \overline{3} c 1$, instead of $P 6_{3} \mathrm{~cm}$, could also be used to refine the experimental data of the distorted structure with similar reliability factors. In some cases the experimental data were not sufficient to distinguish between the two models and only an arbitrary choice between the two

\section{Table 11}

Polarization vectors of the $\mathrm{K} 3, \mathrm{~K} 1$ and GM2- distortions present in the $\mathrm{Pb}_{3} \mathrm{~cm}$ structure of $\mathrm{TlCoCl}_{3}$ (Nishiwaki et al., 2006).

The asymmetric unit is equivalent to that of Table 8 . Displacements are given in relative units with respect to the experimental unit cell $(a=11.86, b=11.86$, $c=5.98 \AA$ ). Polarization vectors are normalized to $1 \AA$.

\begin{tabular}{|c|c|c|c|c|}
\hline \multirow[b]{2}{*}{ Atom } & \multirow{2}{*}{$\frac{\mathrm{K} 3}{\delta \mathrm{z}}$} & \multicolumn{2}{|l|}{ K1 } & \multirow{2}{*}{$\frac{\mathrm{GM} 2-}{\delta \mathrm{z}}$} \\
\hline & & $\delta \mathrm{x}$ & $\delta \mathrm{y}$ & \\
\hline $\mathrm{Co} 1$ & -0.0488 & 0.0000 & 0.0000 & 0.0164 \\
\hline Co1_2 & 0.0244 & 0.0000 & 0.0000 & 0.0164 \\
\hline Tl1 & 0.0000 & -0.0186 & 0.0000 & -0.0611 \\
\hline $\mathrm{Cl} 1$ & -0.0481 & 0.0047 & 0.0000 & 0.0149 \\
\hline Cl1_2 & 0.0240 & 0.0093 & 0.0232 & 0.0149 \\
\hline
\end{tabular}

models could be made (Cussen \& Battle, 2000; Nishiwaki et al., 2006). In fact, as easily checked with SYMMODES (Capillas et al., 2003) or ISOTROPY (Stokes \& Hatch, 2002), this alternative symmetry for the distorted phase would also correspond to a distortion $\mathrm{K} 3$ as primary mode, and $\mathrm{K} 1$ as secondary. The difference with the $P 6_{3} \mathrm{~cm}$ symmetry break would be associated with a change in the direction of the order parameter $\mathrm{K} 3$ in its two-dimensional irrep space, i.e. a different (orthogonal) linear combination of the two independent modes of this symmetry. This would be sufficient to change the symmetry to $P \overline{3} c 1$, and cancel the possibility of having a secondary polar distortion. The fact that the structure of this secondary polar distortion, only present under the hexagonal symmetry, is quite comparable in all the compounds (see Table 10) is a significant factor that should favor the $\mathrm{Pb}_{3} \mathrm{~cm}$ model as the most plausible one.

Another illustration of the insight that the mode decomposition can provide is the comparison of the structure of $\mathrm{BaMnO}_{3}$ at $80 \mathrm{~K}$, which has its mode decomposition summarized in Table 10, with the structure of the same compound at $1.7 \mathrm{~K}$, reported in the same work (Cussen \& Battle, 2000). The amplitudes (in $\AA$ ) of the three irrep distortions of K3, K1 and GM2- symmetries at the lower temperature are $0.53,0.15$ and 0.14 , respectively, to be compared with $0.42,0.04$ and 0.14 for the structure at $80 \mathrm{~K}$ (see Table 10). The distortion amplitudes have increased with the temperature decrease as one would expect, but their polarization vectors have some clear inconsistent variation. Their scalar product with those at $80 \mathrm{~K}$ gives $0.9998,-0.90$ and 0.996 , respectively. Hence, the distortion K1 keeps its internal structure similar to that at $80 \mathrm{~K}$, but has its sign switched, while the other two distortions are practically invariant except for its amplitude increase. From the discussion above it should be clear that the K1 distortion has its sign fixed by that of the primary mode [see equation (14)], and a change of sign of this irrep distortion without a change of sign of the $\mathrm{K} 3$ distortion describes a structure quite different from that reported at $80 \mathrm{~K}$. Therefore, we have here most probably another example of a defective structural model caused by a false refinement minimum with a secondary irrep distortion having a spurious switch of sign. As the amplitude of the K1 mode is significantly larger at $1.7 \mathrm{~K}$, the incorrect sign of this distortion is most probably the one at $80 \mathrm{~K}$. This could also be inferred from 
Table 12

Mode decomposition of the $P 6_{3}$ phases of different $A B X_{3}$ compounds compared with the mode decomposition of the $P 6_{3} \mathrm{~cm}$ phase of $\mathrm{KNiCl}_{3}$.

The third column indicates the direction of the irrep distortion in the irrep space. For each compound the first column shows the amplitude of each irrep distortion, while the second indicates the value of the scalar product of its polarization vector with that of the corresponding distortion in $\mathrm{KNiCl}_{3}$, if existing. $\mathrm{For}_{\text {irrep }}$ distortions only present in the $\mathrm{Pb}_{3}$ configuration, only their amplitudes are indicated. The structural models have been taken from Visser $e t$ al. (1980) for $\mathrm{KNiCl}_{3}$ and Jongen et al. (2005) for the rest.

\begin{tabular}{|c|c|c|c|c|c|c|c|c|c|c|c|c|}
\hline \multirow{2}{*}{$\frac{\mathrm{K} \text {-vector }}{(0,0,0)}$} & \multirow{2}{*}{$\begin{array}{l}\text { Irrep } \\
\text { GM2- }\end{array}$} & \multirow{2}{*}{$\begin{array}{l}\text { Direction } \\
\text { (a) }\end{array}$} & \multirow{2}{*}{$\begin{array}{l}\begin{array}{l}\text { Isotropy } \\
\text { subgroup }\end{array} \\
\mathrm{Pb}_{3} \mathrm{mc}\end{array}$} & \multirow{2}{*}{$\begin{array}{l}\text { Dimension } \\
3\end{array}$} & \multicolumn{2}{|c|}{$\mathrm{KNiCl}_{3}$} & \multicolumn{2}{|c|}{$\begin{array}{l}\mathrm{KTiCl}_{3} \\
w R=0.10\end{array}$} & \multicolumn{2}{|c|}{$\begin{array}{l}\mathrm{KTiBr}_{3} \\
w R=0.15\end{array}$} & \multicolumn{2}{|c|}{$\begin{array}{l}\mathrm{KTiI}_{3} \\
w R=0.16\end{array}$} \\
\hline & & & & & 0.21 & 1 & 0.10 & -0.9992 & 1.21 & -0.63 & 0.81 & 0.65 \\
\hline$(1 / 3,1 / 3,0)$ & $\mathrm{K} 1$ & $(a, 0)$ & $\mathrm{PG}_{3} / \mathrm{mcm}$ & 3 & 0.07 & 1 & 0.18 & 0.97 & 1.09 & 0.90 & 0.54 & -0.91 \\
\hline$(0,0,0)$ & GM2+ & (a) & $P 6_{3} / m$ & 1 & 0 & & 0.74 & & 1.49 & & 2.04 & \\
\hline$(1 / 3,1 / 3,0)$ & $\mathrm{K} 2$ & $(a, 0)$ & $P 6_{3} 22$ & 1 & 0 & & 0.01 & & 0.18 & & 0.02 & \\
\hline$(1 / 3,1 / 3,0)$ & K4 & $(a, 0)$ & $P 6_{3} / m$ & 4 & 0 & & 0.11 & & 2.10 & & 0.76 & \\
\hline
\end{tabular}

Table 10, where the decomposition can be compared with that of $\mathrm{KNiCl}_{3}$.

Further consideration of the hexagonal $A B X_{3}$ compounds that exhibit a distorted structure of even lower symmetry is required. $\mathrm{KTiCl}_{3}, \mathrm{KTiBr}_{3}$ and $\mathrm{KTiI}_{3}$ are reported to have a distorted hexagonal $2 H$ perovskite structure with space group $P 6_{3}$. The three structures have been obtained from singlecrystal X-ray diffraction and reported in a recent publication (Jongen et al., 2005). The reliability factors are, however, rather poor, the weighted $R$ factors being $0.10,0.15$ and 0.16 for $\mathrm{KTiCl}_{3}, \mathrm{KTiBr}_{3}$ and $\mathrm{KTiI}_{3}$, respectively. Clearly, the structural model for $\mathrm{KTiCl}_{3}$ is much more reliable than for the other two compounds. One can perform a mode decomposition of the three structures similar to that done for the $\mathrm{Pb}_{3} \mathrm{~cm}$ compounds. The transformation matrix relating the lattice and origin of these $P 6_{3}$ compounds with that of the hexagonal $2 \mathrm{H}$ perovskite is the same as for the group $P 6_{3} \mathrm{~cm}$. Hence the space group of these compounds is a subgroup of $\mathrm{Pb}_{3} \mathrm{~cm}$ observed in the compounds discussed previously. Fig. 11 shows a graph of maximal subgroups relating the parent and distorted symmetries in this case. The number of irrep distortions permitted by the $P 6_{3} \mathrm{~cm}$ symmetry is enlarged with

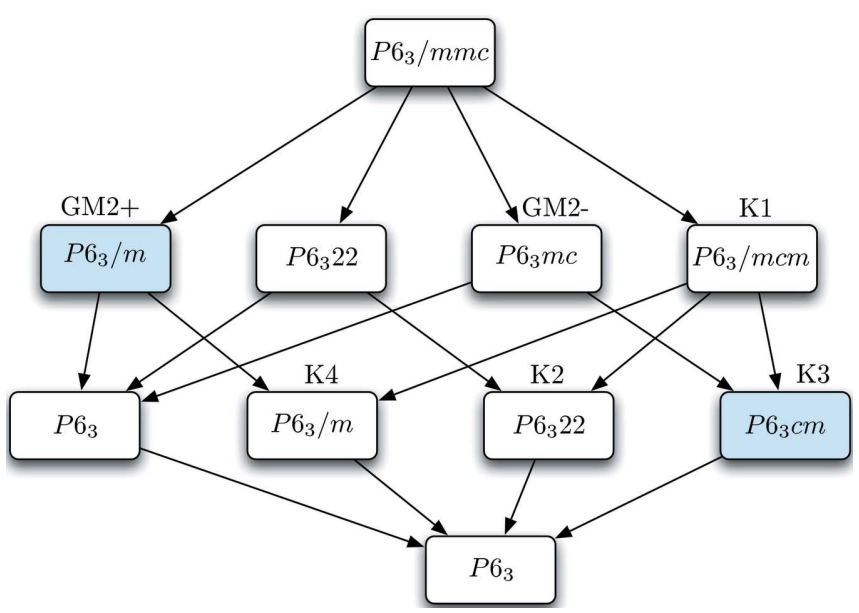

Figure 11

Graph of maximal subgroups relating the space groups of the parent and distorted $\mathrm{P6}_{3}$ phase of $\mathrm{KTiCl}_{3}$. For each subgroup any irrep yielding this symmetry is indicated. The two primary active irreps evidenced by the mode decomposition are highlighted. new irrep components associated with other intermediate symmetries. The most important point evidenced by Fig. 11 is the fact that there is no single irrep distortion that can produce the symmetry break between the parent and the distorted symmetry, i.e. the distortion present in these $P 6_{3}$ phases must have more than one primary distortion. From Fig. 11 it is clear that at least two distortions corresponding to two different irreps are necessary to explain the symmetry break. There could be many pairs of irrep distortions which could be responsible for the observed symmetry $P 6_{3}$, but, assuming that the irrep K3 is also a primary distortion in these compounds, the second primary mode could only be either GM2+, K4 or $\mathrm{K} 2$. The presence of any one of these three distortions together with the $\mathrm{K} 3$ distortion would be sufficient for explaining the observed $\mathrm{Pb}_{3}$ symmetry. The mode decomposition of these three structures summarized in Table 12 permits the identification of the distortion GM2+ as the second primary distortion. It is clearly the dominant component of the distortion that superposes to those yielding the $P 6_{3} \mathrm{~cm}$ symmetry. This distortion is represented in Fig. 12. Its polarization vector is fully determined by symmetry, as only one basis symmetry mode is involved. It is a rotation of the octahedral $B X_{3}$ columns around the $z$ direction. As it is a primary distortion, a switch of the sense of these rotations independently of the sign of the other primary distortion $\mathrm{K} 3$ yields an equivalent configuration. Fig. 11 shows that the secondary distortions K2 and K4 must be induced by the simultaneous presence of both primary modes, as their isotropy subgroups are not supergroups of any of the two primary symmetries $P 6_{3} \mathrm{~cm}$ or $P 6_{3} / \mathrm{m}$ but of their intersection $P 6_{3}$. Indeed, using the module INVARIANTS from ISOTROPY (Stokes \& Hatch, 2002) it can be checked that the lowest coupling of the distortions of symmetry $\mathrm{K} 2$ and $\mathrm{K} 4$ with the primary modes which are responsible for their induction in the distorted phase are $A_{\mathrm{K} 3} A_{\mathrm{GM} 2+} A_{\mathrm{K} 2}$ and $A_{\mathrm{K} 3}^{2} A_{\mathrm{GM} 2+} A_{\mathrm{K} 4}$, respectively. Both distortions are therefore sensitive to the sign of the GM2+ distortion, while only $\mathrm{K} 2$ would also switch with a change of sign of the $\mathrm{K} 3$ distortion.

The K3, K1 and GM2- distortions present in these three $\mathrm{Pb}_{3}$ structures are compared in Table 12 with those of the $P 6_{3} \mathrm{~cm}$ phase of $\mathrm{KNiCl}_{3}$. In the case of $\mathrm{KTiCl}_{3}$, the coincidence of the polarization vectors of the three distortions is striking, despite the quite different amplitudes. To be noted is 
the correlated change of sign of the $\mathrm{K} 3$ and GM2 - distortions, corresponding to an equivalent domain-related configuration. On the other hand, the mode decomposition of the other two compounds, with much poorer reliability factors, suggests where the problems of these structural models could be. While the polarization vector of the primary mode $\mathrm{K} 3$, given by a single parameter, is essentially the same as in $\mathrm{KNiCl}_{3}$, the secondary ones have much larger relative amplitudes than in $\mathrm{KTiCl}_{3}$ and their three-dimensional polarization vectors have erratic changes of sign, not even consistent between the two. If $\mathrm{KTiI}_{3}$ and $\mathrm{KTiBr}_{3}$ are compared, their distortions GM2- and $\mathrm{K} 1$ are very similar but their signs are switched, and do not correspond to equivalent domain-related configurations. These structures are probably also associated with false refinement minima for structures with switched secondary modes.

The mode decomposition of these $P 6_{3} A B X_{3}$ structures also helps to infer the probable temperature behavior of these compounds. If, as usual, they acquire the parent symmetry $\mathrm{P6}_{3} / \mathrm{mmc}$ at high temperatures, one can expect the existence of an intermediate $\mathrm{Pb}_{3} \mathrm{~cm}$ phase after the second primary mode $\mathrm{GM} 2+$ is thermalized. One cannot discard of course a single first-order phase transition with both order parameters

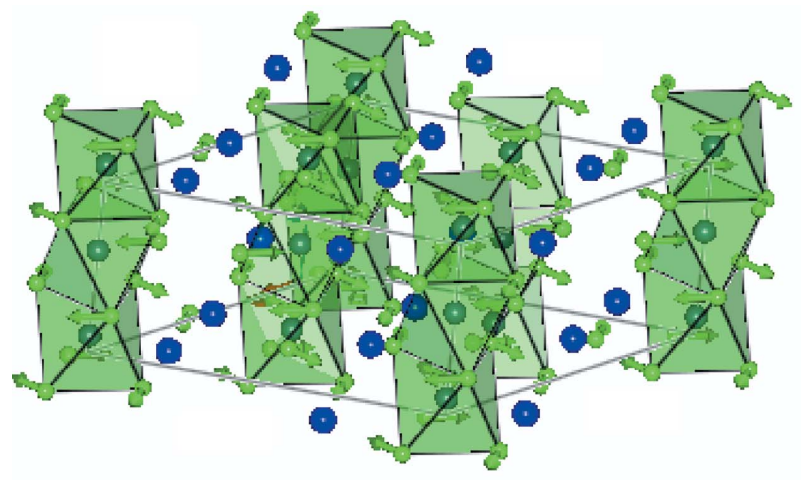

(a)

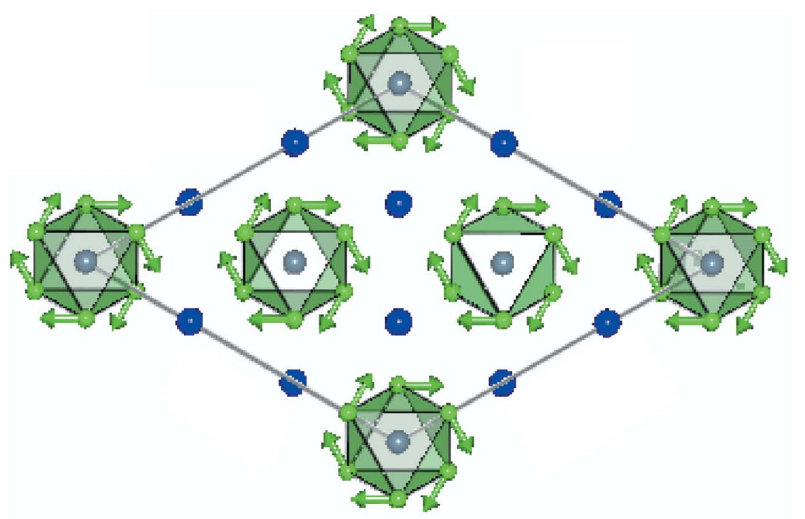

(b)

Figure 12

Polarization vector of the primary distortion GM2+ present in the $\mathrm{PG}_{3}$ structures of $\mathrm{KTiCl}_{3}, \mathrm{KTiBr}_{3}$ and $\mathrm{KTiI}_{3}$. (a) Perspective view, (b) projection on the plane $x y$. The scale of the displacement vectors has been enlarged. [Figure created using FullProf Studio (Rodríguez-Carvajal, 1993).] becoming zero simultaneously, but in most cases two active primary irrep distortions imply two successive symmetry breaks.

\section{Distorted pseudocubic perovskites: sequence of phase transitions}

There are many $A B X_{3}$ structures having as parent structure the cubic perovskite with space group $P m \overline{3} m$. Depending on the so-called Goldsmidt or tolerance factor which quantifies the misfit of the sizes of the three ions, different distorted structures exist, and the cubic parent phase is often reached at high temperatures after following some sequence of phase transitions. For these simple structures with rather rigid $B X_{6}$ octahedra a mode description is quite simple; many normal modes are fully determined by symmetry and can be identified with tilts or RUMs (Giddy et al., 1993; Hammonds et al., 1996) of the framework of octahedra. A good deal of the static distortions present in these compounds can in fact be described as tilting schemes of the $B X_{6}$ octahedra, and have been rationalized from this viewpoint (Glazer, 1972; Woodward, 1997a,b). The more general approach of a mode description has also been considered (Howard \& Stokes, 1998; Darlington, 2002; Knight, 2009). It is not the aim of this section to review such an extensive subject. We only want to present a few cases within this family as further examples of the power of a systematic mode decomposition.

Let us consider the very well studied case of $\mathrm{SrZrO}_{3}$, which has at room temperature a distorted perovskite structure with Pnma symmetry (Howard et al., 2000; Kennedy, Howard \& Chakoumakos, 1999, and references therein), which is typical of many $A B X_{3}$ compounds having an $A$ cation too small to stabilize the cubic configuration. In this compound the cubic perovskite structure is only attained above $1340 \mathrm{~K}$. The setting of its orthorhombic Pnma space group is related to the supergroup $P m \overline{3} m$ corresponding to its parent structure by the transformation $(\mathbf{a}+\mathbf{c}, 2 \mathbf{b},-\mathbf{a}+\mathbf{c} ; 0,0,0)$. The distortion mainly involves correlated tilting of the $\mathrm{ZrO}_{6}$ octahedra, i.e. RUMs of the octahedral framework (see Fig. 13), with a multiplication of the unit cell by a factor of four. This implies the presence of a considerable number of distortion modes of different symmetry compared with the examples above. On the other hand, the number of free atomic coordinates is quite limited. Fig. 14 shows the graph of maximal subgroups connecting the two space groups and, if existing, the irrep yielding these intermediate symmetries as isotropy subgroups. It can be seen that distortions with symmetries given by irreps corresponding to three different symmetry points at the border of the cubic Brillouin zone will be present in the Pnma structure, namely the points $\mathrm{M}(1 / 2,1 / 2,0), \mathrm{X}(0,1 / 2,0)$ and $\mathrm{R}$ $(1 / 2,1 / 2,1 / 2)$. Furthermore, the graph shows that the Pnma symmetry of this phase is not an isotropy subgroup of $P m \overline{3} m$, i.e. this symmetry cannot be attained with a single primary mode. At least two primary modes are necessary. In other words, the Pnma phase cannot be generated by a single mechanism or a single unstable mode of the cubic configuration, but at least two different normal modes must be active. In 
Table 13

Summary of the mode decomposition with respect to its $\operatorname{Pm} \overline{3} m$ parent structure of the Pnma structure of $\mathrm{SrZrO}_{3}$ at $293 \mathrm{~K}$ (Howard et al., 2000), $\mathrm{NaTaO}_{3}$ (Kennedy, Prodjosantoso \& Howard, 1999) and $\mathrm{LaMnO}_{3}$ at 300 K (Rodríguez-Carvajal et al., 1998).

\begin{tabular}{llllll}
\hline & \multirow{2}{*}{$\begin{array}{l}\text { Isotropy } \\
\text { Irrep }\end{array}$} & subgroup & Dimension & \multicolumn{3}{l}{ Amplitude $(\AA)$} \\
\cline { 4 - 6 } & $\mathrm{SrZrO}_{3}$ & $\mathrm{NaTaO}_{3}$ & $\mathrm{LaMnO}_{3}$ \\
\hline $\mathrm{R} 4+$ & Imma & 1 & 1.19 & 0.97 & 1.19 \\
$\mathrm{R} 5+$ & Imma & 2 & 0.07 & 0.03 & 0.09 \\
$\mathrm{X} 5+$ & $\mathrm{Cmcm}$ & 2 & 0.34 & 0.23 & 0.56 \\
$\mathrm{M} 2+$ & $P 4 / m b m$ & 1 & 0.01 & 0.01 & 0.36 \\
$\mathrm{M} 3+$ & $P 4 / m b m$ & 1 & 0.79 & 0.78 & 0.90 \\
\hline
\end{tabular}

the language of Landau theory, the phase Pnma should be the result of the condensation of two order parameters. These order parameters are in general expected to be thermalized and become zero at higher temperatures, but each one independently, producing two phase transitions. Thus, one can expect from this simple symmetry relation regarding the parent and distorted space groups the probable presence of an intermediate phase before the system reaches the cubic perovskite.

From the graph in Fig. 14 one can establish the different possible primary distortions that may be relevant. One has to look for pairs of isotropy subgroups which have as intersection the observed space group Pnma. There are many possibilities. Any pair of the distortions indicated in the graph, except for the pair of the two M modes or the pair of two R modes, would be sufficient to explain the observed Pnma symmetry.

A mode decomposition of the experimental structure at $293 \mathrm{~K}$ (Howard et al., 2000) clearly shows which are the primary distortions. Table 13 lists the amplitudes of all the distortion modes, and one can clearly see that two distortion amplitudes are much larger, namely those of the distortions $\mathrm{R} 4+$ and $\mathrm{M} 3+$, the one for R4+ being significantly larger. A

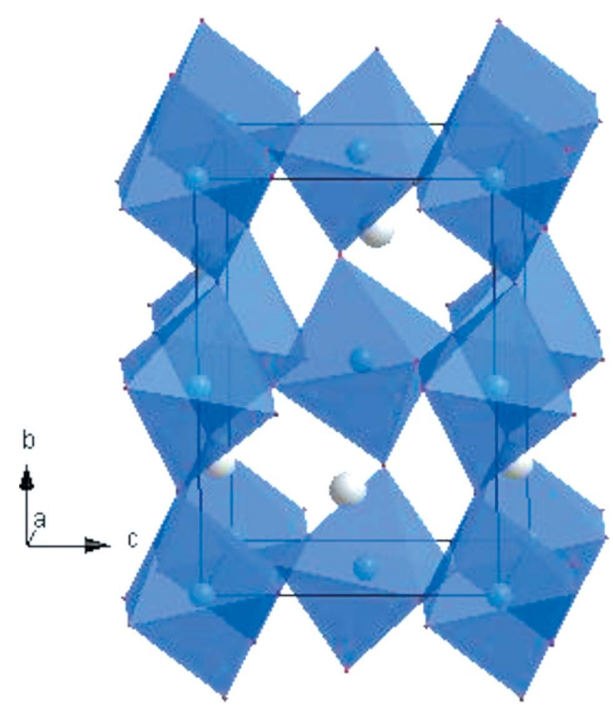

Figure 13

Structure of the Pnma phase of $\mathrm{SrZrO}_{3}$, showing the strong tilting of the octahedra with respect to the parent perovskite.

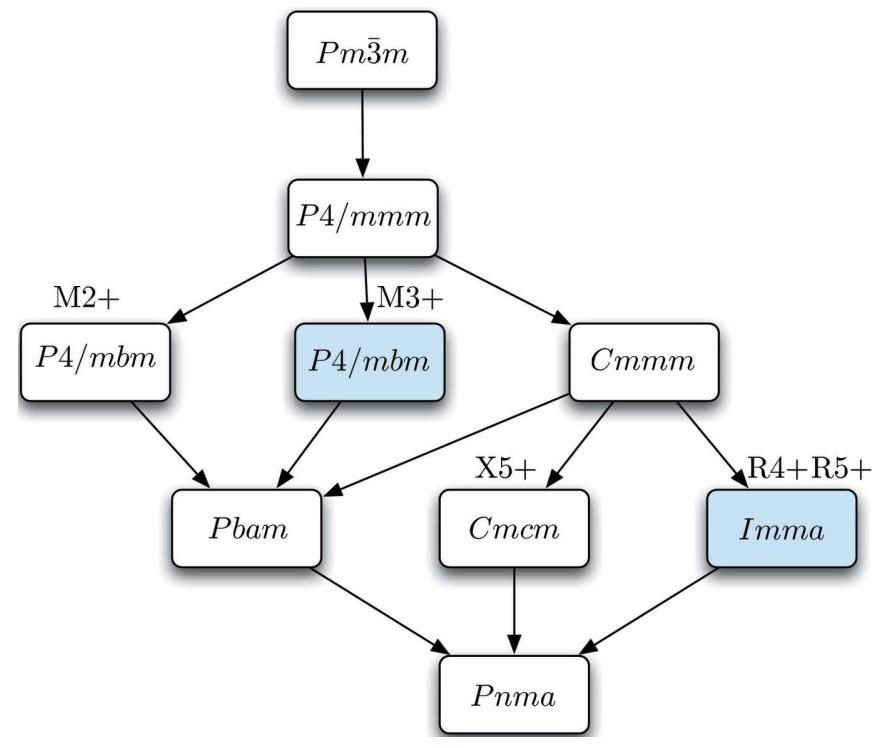

Figure 14

Graph of maximal subgroups relating the space groups of the parent and the Pnma phase of $\mathrm{SrZrO}_{3}$. For each subgroup any irrep yielding this symmetry is indicated. The subgroups corresponding to the two primary distortions are highlighted.

scheme of the five distortion modes participating in the Pnma structure can be seen in Fig. 15. Their polarization vectors are listed in Table S1 of the supplementary material. ${ }^{7}$ The two primary distortion modes are tilting modes of the octahedra with a single basis mode involved, and therefore fully determined by symmetry. The secondary mode X5+, however, involves both oxygen and $\mathrm{Sr}$ displacements, and despite implying some distortion of the octahedra it has a significant non-zero amplitude. The two remaining secondary distortion modes are very weak. The M2+ is zero within experimental resolution, while the R5+ distortion, although very small, is present in the structure, and mainly involves displacements of the $\mathrm{Sr}$ atoms along the orthorhombic $z$ direction.

Distortion modes of symmetry R4+ and M3+ are therefore the two dominant primary distortion modes underlying the Pnma structure of $\mathrm{SrZrO}_{3}$. An analogous symmetry-mode decomposition in other compounds shows that this in fact happens in most of the distorted Pnma perovskites, with the R4+ distortion often being the strongest (Knight, 2009). In this simple case these primary modes are defined in onedimensional spaces and their polarization vectors are fully determined by symmetry, corresponding to simple so-called tilt systems of the octahedra (Glazer, 1972; Howard \& Stokes, 1998). Only their amplitudes are variable, and for small values these amplitudes are linearly related to the corresponding tilt angle.

The identification of these tilt sytems as the two primary modes, with a symmetry given by an irrep of the parent space group $P m \overline{3} m$, is information directly obtained from the

\footnotetext{
${ }^{7}$ These polarization vectors and the output of $A M P L I M O D E S$ with the detailed mode decomposition for all examples discussed in this article are available from the IUCr electronic archives (Reference: SH5107). Services for accessing these data are described at the back of the journal.
} 
structure, which is very valuable for inferring possible transition sequences, and general trends in the whole family. In fact, as pointed out in previous literature (Howard \& Stokes, 1998), the instability of the perovskite cubic configuration with respect to RUMs of symmetry R4+ and M3+ underlies many of the distorted phases with various symmetries. The R4+ RUM modes correspond for instance to the well known (threefold degenerate) instability present in $\mathrm{SrTiO}_{3}$ which competes with the ferroelectric one (Zhong \& Vanderbilt, 1995, and references therein), and yields for this compound at low temperatures a tetragonal phase with $14 / \mathrm{mcm}$ symmetry. ${ }^{8}$ Both irreps $\mathrm{R} 4+$ and $\mathrm{M} 3+$ are three-dimensional and the distortion and symmetry that is realized in $\mathrm{SrZrO}_{3}$ corresponds to specific directions within the space of each representation indicated symbolically in Table 13. An extended general explanation of the meaning of specific directions of a distortion mode within the irrep space and their relations with the isotropy subgroup can be found in Hatch \& Stokes (1985) and Howard \& Stokes (1998). In the present case, changing the direction within the irrep space means in general a change of the axis around which the tilts of the octahedra take place, with a consequent change of the resulting (isotropy) space group. For instance, the possible symmetries for an R4+ distortion are given by the following isotropy subgroups, ${ }^{9}$

(a) I4/mcm, $(\mathbf{a}+\mathbf{b},-\mathbf{a}+\mathbf{b}, 2 \mathbf{c} ; 0,0,0),(a, 0,0)$,

(b) Imma, (a + c, 2b, -a + c; 0, 0, 0), (a, a, 0),

(c) $R \overline{3} c,(-\mathbf{a}+\mathbf{b},-\mathbf{b}+\mathbf{c}, 2 \mathbf{a}+2 \mathbf{b}+2 \mathbf{c} ; 0,0,0),(a, a, a)$,

(d) $C 2 / m,(-2 \mathbf{c}, 2 \mathbf{b}, \mathbf{a}+\mathbf{c} ; 0,1 / 2,1 / 2),(a, b, 0)$,

(e) $C 2 / c,(-\mathbf{a}+2 \mathbf{b}-\mathbf{c},-\mathbf{a}+\mathbf{c}, \mathbf{a}+\mathbf{c} ; 0,1 / 2,1 / 2),(a, a, b)$,

(f) $P \overline{1},(\mathbf{b}+\mathbf{c}, \mathbf{a}+\mathbf{c}, \mathbf{a}+\mathbf{b} ; 0,0,0),(a, b, c)$.

The last item in each row indicates for each case the direction of the distortion in the irrep space, in the notation used by ISOTROPY (Stokes \& Hatch, 2002).

For a given R4+ instability of the cubic perovskite, the realization of one or other space group of the above list depends on the anharmonic terms in the free-energy function discussed in $\S 4$, which creates the anisotropy of the energy map in the three-dimensional subspace defined by the R4+ unstable threefold degenerate distortion modes. Usually, because of the smoothness of the energy map, the energy minima will correspond to high-symmetry directions within the map (Vanderbilt \& Cohen, 2001). These free-energy minima can change with temperature and a sequence of firstorder phase transitions then happens, with symmetry changes between different isotropy subgroups of the same irrep. This is observed for instance in $\mathrm{CeAlO}_{3}$, where only $\mathrm{R} 4+$ distortions act as primary within its phase-transition sequence for increasing temperature (Fu \& Ijdo, 2006; Avdeev et al., 2007),

$$
I 4 / m c m-I m m a-R \overline{3} c-P m \overline{3} m .
$$

\footnotetext{
${ }^{\mathbf{8}}$ The irreps labels do not necessarily coincide with those used in other studies (Darlington, 2002). Unfortunately, even keeping a fixed specific choice of notation, the irrep label may change depending on the origin choice in the parent structure. For instance, if the origin is chosen at the site of the $\mathrm{Sr}$ atom instead of the $\mathrm{Zr}$, the irrep label of the distortion $\mathrm{R} 4+$ would change to R5-. ${ }^{9}$ They can be obtained with ISODISPLACE (Campbell et al., 2006) and they are also listed in Howard \& Stokes (1998).
}

The phase transitions are associated with changes of direction of the R4+ order parameter (distortion) according to the list of isotropy subgroups above. This is fully analogous to the consecutive discontinuous phase transitions taking place in $\mathrm{BaTiO}_{3}$, owing to changes of the direction of its spontaneous polarization, directly related to changes of direction of its polar GM4- distortion within its three-dimensional irrep space (see $\S 3$ ).

In the case of $\mathrm{SrZrO}_{3}$, the $\mathrm{R} 4+$ distortion corresponds to the direction $(a, a, 0)$ with symmetry Imma. It is namely a combination of two equal tilts around the $[1,0,0]$ and $[0,0,1]$ cubic perovskite axes, which is equivalent to a tilt around the oblique direction $[1,0,1]$, i.e. around the $x$ direction of the orthorhombic setting (see Fig. 15).

As the R4+ distortion is clearly much stronger than the M3+ distortion, one can infer that this latter will be thermalized at lower temperatures leaving a phase with only R4+ as primary distortion. If the direction of the R4+ distortion mode does not change, one can predict a phase transition into a phase with Imma as space group, which can in principle be continuous. If temperature is further increased, subsequent transitions corresponding to changes of direction of the primary $\mathrm{R} 4+$ distortion mode may happen, until the cubic phase is finally reached. Indeed, this is what happens in $\mathrm{SrZrO}_{3}$, with a reported transition sequence (Kennedy, Howard \& Chakoumakos, 1999)

$$
\text { Pnma - Imma }-I 4 / m c m-P m \overline{3} m .
$$

Therefore, the relative weight of several primary distortion modes in a distorted structure can give important clues concerning its behavior at higher temperatures. We can
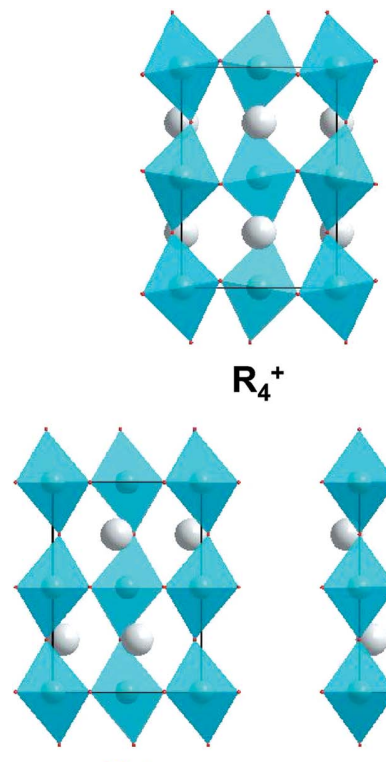

$\mathrm{X}_{5}^{+}$

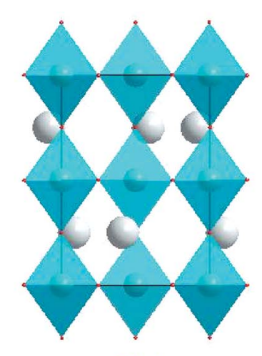

$\mathbf{R}_{5}{ }^{+}$

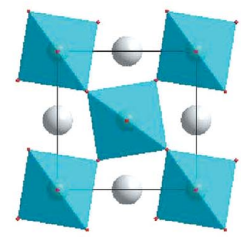

$\mathrm{M}_{3}{ }^{+}$

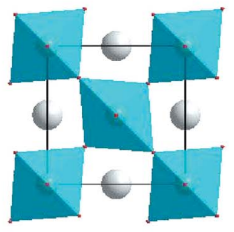

$\mathrm{M}_{2}^{+}$

\section{Figure 15}

Scheme of the polarization vectors of the five different irrep distortion components present in the Pnma structure of $\mathrm{SrZrO}_{3}$. The figure shows in each case the distorted structure for an arbitrary amplitude of the distortion. 
Table 14

Summary of the mode decomposition of the $\mathrm{Cmcm}$ and $\mathrm{P} 4 / \mathrm{mbm}$ phases of $\mathrm{NaTaO}_{3}$.

\begin{tabular}{llll}
\hline Irrep & $\begin{array}{l}\text { Isotropy } \\
\text { subgroup }\end{array}$ & Dimension & Amplitude $(\AA)$ \\
\hline Phase $C m c m$ & & & \\
R4+ & $I 4 / \mathrm{mcm}$ & 1 & 0.49 \\
R5+ & $I 4 / \mathrm{mmm}$ & 2 & 0.04 \\
$\mathrm{X} 5+$ & $P m m a$ & 2 & 0.12 \\
M3+ & $P 4 / \mathrm{mbm}$ & 1 & 0.54 \\
M4+ & $P 4 / \mathrm{mmm}$ & 1 & 0.01 \\
Phase $P 4 / \mathrm{mbm}$ & & & \\
M3+ & $P 4 / \mathrm{mbm}$ & 1 & 0.38 \\
\hline
\end{tabular}

crosscheck this by comparing the mode decomposition of $\mathrm{SrZrO}_{3}$ with the analogous phase of $\mathrm{NaTaO}_{3}$ (Kennedy, Prodjosantoso \& Howard, 1999), also shown in Table 13. In this compound the amplitude of the R4+ distortion is about $20 \%$ smaller, while the M3+ distortion is of the same order of magnitude. Although the R4+ distortion is still the largest, its amplitude is much closer to that of $\mathrm{M} 3+$. In this case the transition sequence is quite different,

$$
P n m a-C m c m-P 4 / m b m-P m \overline{3} m .
$$

The mode decomposition of these high-temperature phases is shown in Table 14. It can be seen that the $\mathrm{Cmcm}$ phase is also the result of the presence of the two distortion modes with irrep symmetry $\mathrm{R} 4+$ and $\mathrm{M} 3+$, but the R4+ distortion has changed its direction, so that now its isotropy subgroup is $I 4 / \mathrm{mcm}$. Its amplitude is now significantly smaller than that of the M3+ distortion. One can then infer, as it is indeed the case, that the subsequent phase $P 4 / \mathrm{mbm}$ must be caused only by the presence of the $\mathrm{M} 3+$ distortion, with the $\mathrm{R} 4+$ distortion thermalized at a lower temperature than the $\mathrm{M} 3+$ distortion, the opposite of what happens in $\mathrm{SrZrO}_{3}$.

The M2+ distortion, which distorts the $B X_{6}$ octahedra (see Fig. 15 and Table 1 in the supplementary material), is practically negligible both in $\mathrm{SrZrO}_{3}$ and $\mathrm{NaTaO}_{3}$, but can have important amplitudes in the Pnma phase of perovskites with Jahn-Teller ions. The local symmetry of the octahedral distortions associated with this mode corresponds to that induced by the local Jahn-Teller effect (Carpenter \& Howard, $2009 a, b)$. Table 13 shows the mode decomposition of $\mathrm{LaMnO}_{3}$ (Rodríguez-Carvajal et al., 1998), where the presence of a significant $\mathrm{M} 2+$ distortion is patent, in contrast with the previous examples. Despite its compatibility with the symmetry produced by the two primary dominant distortions $\mathrm{R} 4+$ and $\mathrm{M} 3+$, the M2+ distortion in Jahn-Teller Pnma perovskites act as a third primary mode instead of as a secondary induced distortion. In fact, this additional primary mode usually introduces a new phase transition corresponding to its independent condensation. As the Pnma symmetry is compatible with the distortion, this additional Jahn-Teller transition would not represent any symmetry change in a structure with both R4+ and M3+ already frozen, and an isosymmetrical Pnma-Pnma transition takes place, with a conspicuous increase of the amplitude of the M2+ distortion acting as a non-symmetry-breaking order parameter. This is what happens in $\mathrm{LaMnO}_{3}$ at $750 \mathrm{~K}$ (Rodríguez-Carvajal et al., 1998).

\section{Distorted structures as commensurate modulated structures: mode decomposition versus superspace description}

The description of commensurately distorted structures in terms of symmetry-adapted distortion modes is closely related to the alternative approach of treating them as modulated structures with the use of the superspace formalism (PerezMato, 1991; Janssen et al., 2004). Displacive distortion modes are in fact modulations with the wavevectors associated with their corresponding irrep. Thus, in the previous example of $\mathrm{SrZrO}_{3}$, three modulations with wavevectors $(1 / 2,1 / 2,0)$, $(0,0,1 / 2)$ and $(1 / 2,1 / 2,1 / 2)$ are present.

To describe a commensurately distorted structure as a modulated phase a set of primary modulation wavevectors is defined and the distortion is given by a superposition of harmonics of this set of primary wavevectors. The symmetry is given by a superspace group, which defines the correlations and symmetry restrictions that the atomic displacements must have for each of these harmonics. In the case of incommensurate structures the number of harmonics is unlimited, but in practice a hierarchy exists among them and the first harmonics are expected to be dominant, so that the expansion can be truncated. In a commensurate case the number of possible harmonics is finite, and a hierarchy between first and higher harmonics also exists, so that in some cases the highest harmonics can be neglected. Under these premises the program JANA (Petricek et al., 2006), for instance, is adapted to treat and refine, using the superspace formalism, any commensurately distorted structure with up to three independent primary modulation wavevectors.

In simple cases, a mode decomposition in terms of irrep distortion modes and a decomposition with modulation harmonics under a postulated superspace group are fully equivalent, i.e. each irrep distortion corresponds to a specific harmonic in the modulation, and the secondary modes can in general be identified with higher-order harmonics in the superspace description. This happens when the average space group in the superspace group is that of the parent structure and a single primary wavevector exists, so that the modulation is one-dimensional. In more complex cases the two methods may differ in the way they decompose the global distortion. In general, if the unit cell of the distorted phase is much larger than the parent one, a mode decomposition would not bring much benefit to what is already provided by the superspace approach, and would be somehow less efficient, since the structure of the polarization vectors can be trivially derived from the modulation wavevector. On the other hand, for supercells in the distorted phase, which are only a small multiple of the parent phase, and involve modulations along several directions, the mode approach is usually more convenient. We illustrate these considerations with some 
Table 15

Summary of the mode decomposition of the $P \overline{1}$ phase of $\mathrm{NbS}_{3}$ (Rijnsdorp \& Jellinek, 1978).

\begin{tabular}{llllcl}
\hline K-vector & Irrep & Direction & $\begin{array}{l}\text { Isotropy } \\
\text { subgroup }\end{array}$ & Dimension & $\begin{array}{l}\text { Amplitude } \\
(\AA)\end{array}$ \\
\hline$(0,0,0)$ & GM1+ & $(a)$ & $P{ }_{1} / m$ & 8 & $0.000(5)$ \\
$(0,0,0)$ & GM2+ & $(a)$ & $P \overline{1}$ & 4 & $0.036(3)$ \\
$(0,1 / 2,0)$ & $\mathrm{Z} 1$ & $(0, a)$ & $P \overline{1}$ & 12 & $0.520(4)$ \\
\hline
\end{tabular}

Table 16

Summary of the mode decomposition of the threefold lock-in phase of $\mathrm{K}_{2} \mathrm{SeO}_{4}$ (Yamada et al., 1984).

\begin{tabular}{llllrl}
\hline K-vector & Irrep & Direction & $\begin{array}{l}\text { Isotropy } \\
\text { subgroup }\end{array}$ & Dimension & $\begin{array}{l}\text { Amplitude } \\
(\AA)\end{array}$ \\
\hline$(0,0,0)$ & GM1+ & $(a)$ & Pnma & 13 & 0.12 \\
$(0,0,0)$ & GM4- & $(a)$ & Pna2 & 8 & 0.55 \\
$(1 / 3,0,0)$ & SM2 & $(a, 0)$ & Pna2 & 16 & 1.16 \\
$(1 / 3,0,0)$ & SM3 & $(a, 0)$ & Pnma & 26 & 0.39 \\
\hline
\end{tabular}

examples. Further discussion on this matter can also be found by Perez-Mato et al. (2010).

Let us consider first the simple case of the triclinic structure of $\mathrm{NbS}_{3}$. van Smaalen (1988) showed that the triclinic structure of this compound with space group $P \overline{1}$ could be described and refined as a modulated structure with modulation wavevector $(0,1 / 2,0)$, with respect to a basic monoclinic structure having space group $P 2_{1} / m$ and a unit cell with half the volume. The structure could be refined satisfactorily introducing a single harmonic in the modulation, which implied using a smaller number of parameters than a conventional refinement in the triclinic space group $P \overline{1}$. The reason for this can be clearly seen in the mode decomposition of the experimental structure (Rijnsdorp \& Jellinek, 1978), which is summarized in Table 15. The decomposition has been performed with respect to a virtual parent $P 2_{1} / m$ structure symmetrizing the experimental one, such that the GM1+ distortion has been minimized to zero. One can see that the symmetry-breaking distortion has two components: a strong distortion $\mathrm{Z} 1$ with wavevector $q=(0,1 / 2,0)$ yielding the observed symmetry, and a much weaker secondary distortion GM2+ at the Brillouinzone center, with an amplitude more than one order of magnitude smaller. This secondary mode also breaks the binary symmetry, but maintains the lattice of the parent phase. Its weakness is another example of the hierarchy among distinct irrep distortions in distorted structures. In the superspace description this secondary distortion corresponds to a second harmonic with wavevector $2 q$, which for this commensurate case is formally equivalent to $(0,0,0)$, but that the superspace description treats separately (Perez-Mato, 1991). Hence, a superspace refinement of the structure considering only sinusoidal modulations, as performed by van Smaalen (1988), is fully equivalent to a refinement within a model with only the primary distortion $\mathrm{Z} 1$, with the distortion GM2+ being forced to have zero amplitude.

Let us consider now a more complex case in the muchstudied ferroelectric phase of $\mathrm{K}_{2} \mathrm{SeO}_{4}$. This structure has been both analyzed as a modulated phase (Parisi \& Bonadeo, 1997) and in terms of irrep distortion modes (Perez-Mato et al., 1986). It is a commensurate $P n a 2_{1}$ structure, with a triplication of a parent Pnma unit cell, which is the consequence of the lock-in of the modulation wavevector $q=\alpha a^{*}$ of a previous incommensurate phase into the value $\alpha=1 / 3$. It is then natural to describe this structure as a one-dimensional modulated phase with the same superspace group as the incommensurate phase, but with a commensurate wavevector, as done by Parisi \& Bonadeo (1997).

We can, however, also apply the mode decomposition explained above. To use AMPLIMODES we only need to introduce the Pnma (parent) and Pna2 ${ }_{1}$ structures, and the transformation relating both groups: $(-3 \mathbf{a}, \mathbf{c}, \mathbf{b} ; 0,0,0)$. The results are summarized in Table 16. As expected, we have a dominant component for the irrep SM2, with wavevector $(1 / 3,0,0) .{ }^{\mathbf{1 0}}$ This prevailing SM2 distortion is the primary unstable mode that comes from the incommensurate phase through the lock-in of the wavevector. This primary distortion corresponds to the first harmonic of the modulation in the incommensurate phase and determines the superspace-group symmetry governing the symmetry properties of all additional harmonics (Perez-Mato et al., 1984). There is also a weaker distortion with the same wavevector but different irrep, namely SM3. This secondary distortion can be identified with the second harmonic in a modulated description. For a wavevector $q=(1 / 3,0,0)$, the second harmonic distortion has the same wavevector as the first harmonic, but in the superspace approach, as in the previous example, it can be treated as a distinguishable second harmonic of the modulation functions if we use the superspace group of the preceding incommensurate phase. The symmetry properties of the atomic displacements described by the irrep SM3 are then equivalent to those introduced on the second harmonic modulation by this superspace group. Similarly, the additional GM4- distortion is the polar distortion responsible for the spontaneous polarization and ferroelectric properties in this commensurate phase, and can be identified with a third harmonic in the atomic modulations (Perez-Mato et al., 1986; Aramburu et al., 2006). In this example, it is noticeable that the two allowed secondary distortions have smaller but significant amplitudes, so that a satisfactory direct refinement of the structure using either modes or superspace modulation functions requires the same number of positional parameters as a conventional refinement.

As a third example we take the mode decomposition of the ninefold commensurately modulated phase of thiourea $\left[\mathrm{SC}\left(\mathrm{NH}_{2}\right)_{2}\right]$. This structure is an intermediate lock-in phase sandwiched within the extensive range of an incommensurate phase (Moudden et al., 1979). The parent non-modulated structure has Pnma symmetry, and the modulation wavevector is $q=1 / 9 b^{*}$, with therefore a ninefold multiplication of the unit cell. The structure has been refined both as an incommensurately modulated structure using the superspace

\footnotetext{
${ }^{\mathbf{1 0}}$ It is important to stress that, even keeping a fixed standard for the labeling of the irreps, the irrep labels may change for different equivalent choices of the irrep wavevector. One should also take into account the dependence of the irrep label on the setting chosen for describing the parent structure.
} 
Table 17

Summary of the mode decomposition of the ninefold phase of thiourea according to the structure reported as model 1 in Tanisaki et al. (1988) (H atoms not included).

\begin{tabular}{llllcl}
\hline K-vector & Irrep & Direction & $\begin{array}{l}\text { Isotropy } \\
\text { subgroup }\end{array}$ & Dimension & $\begin{array}{l}\text { Amplitude } \\
(\AA)\end{array}$ \\
\hline$(0,0,0)$ & GM1+ & $(a)$ & Pnma & 7 & $0.08(1)$ \\
$(0,1 / 9,0)$ & DT4 & $(a,-5.671 a)$ & Pnma & 12 & $1.89(1)$ \\
$(0,2 / 9,0)$ & DT1 & $(a, 0.364 a)$ & Pnma & 12 & $0.13(1)$ \\
$(0,1 / 3,0)$ & DT4 & $(a,-1.732 a)$ & Pnma & 12 & $0.19(2)$ \\
$(0,4 / 9,0)$ & DT1 & $(a, 0.839 a)$ & Pnma & 12 & $0.09(2)$ \\
\hline
\end{tabular}

approach (Zuñiga et al., 1989) and as a conventional superstructure (Tanisaki et al., 1988). The two models were shown to be approximately equivalent (Perez-Mato, 1991) (see below). Table 17 illustrates the features of the structure refined as a conventional superstructure when decomposed in irrep distortions (the $\mathrm{H}$-atom positions have not been included). A distortion with symmetry given by the irrep DT4 with a wavevector on the line DT $(0, \beta, 0)$ of the Brillouin zone with $\beta=1 / 9$ is dominant. The amplitudes of the additional distortions are between 10 and 20 times smaller so that it can be clearly identified as the primary mode. Note that the four irrep distortions have as isotropy subgroup the observed space group, and therefore from symmetry arguments any of them could be the primary distortion. In this case the primary character of the distortion with $q=1 / 9 b^{*}$ can only be derived from the comparison of the amplitudes of the different irrep distortions. Also, in contrast with $\mathrm{K}_{2} \mathrm{SeO}_{4}$, all irrep distortions have in this example different wavevectors corresponding to distinct harmonics of the primary one. Depending on the parity of the irrep wavevector, the small representation associated with the irrep distortion changes from DT4 to DT1. It is noticeable that the secondary third-order harmonic with wavevector $3 q=(0,1 / 3,0)$, of the same symmetry as the primary distortion, has a larger amplitude than the second one, with a different symmetry. This third harmonic is responsible for the soliton-like form of the atomic modulations in the superspace description (Zuñiga et al., 1989). The modulated refinement in this reference was carried out using only harmonics up to third order for describing the atomic modulations. This means neglecting a possible fourth-order harmonic, which in the mode decomposition corresponds to the weaker DT1 distortion with wavevector $4 q=(0,4 / 9,0)$. Table 17 shows that in the model refined as a conventional superstructure the amplitude of this distortion, although very small, is larger than its standard deviation. This difference is at the origin of the small differences between the atomic positions of the two models, shown by Perez-Mato (1991).

It is interesting to compare in this last example the description of each harmonic modulation obtained by the two approaches. Table 18 shows the polarization vector of the primary DT4 distortion in the mode description. The description of the distortion mode is quite redundant. The three cations $\mathrm{S}, \mathrm{C}$ and $\mathrm{N}$ have a single independent site in the parent unit cell and are split into five, five and nine independent sites, respectively, in the ninefold structure. To describe

\section{Table 18}

Reference structure and polarization vector of the $(0,1 / 9,0)$ DT4 distortion in the ninefold Pnma phase of thiourea according to the structure reported as model 1 of Tanisaki et al. (1988) (H atoms not included) $(a=7.545, b=76.867, c=5.467 \AA)$.

Reference structure

\begin{tabular}{llll}
\hline Atom & $x$ & $y$ & $z$ \\
\hline S1 & 0.9924 & 0.0278 & 0.1147 \\
S1_2 & 0.0076 & 0.0833 & 0.8853 \\
S1_3 & 0.9924 & 0.1389 & 0.1147 \\
S1_4 & 0.9924 & 0.2500 & 0.1147 \\
S1_5 & 0.9924 & 0.6944 & 0.1147 \\
C1 & 0.0881 & 0.0278 & 0.8323 \\
C1_2 & 0.9119 & 0.0833 & 0.1677 \\
C1_3 & 0.0881 & 0.1389 & 0.8323 \\
C1_4 & 0.0881 & 0.2500 & 0.8323 \\
C1_5 & 0.0881 & 0.6944 & 0.8323 \\
N1 & 0.1275 & 0.0425 & 0.7202 \\
N1_2 & 0.8725 & 0.0981 & 0.2798 \\
N1_3 & 0.6275 & 0.0130 & 0.7798 \\
N1_4 & 0.1275 & 0.1536 & 0.7202 \\
N1_5 & 0.1275 & 0.2648 & 0.7202 \\
N1_6 & 0.1275 & 0.3758 & 0.7202 \\
N1_7 & 0.1275 & 0.7092 & 0.7202 \\
N1_8 & 0.1275 & 0.8203 & 0.7202 \\
N1_9 & 0.1275 & 0.9314 & 0.7202 \\
\hline & & &
\end{tabular}

Polarization vector

\begin{tabular}{lrrr}
\hline Atom & \multicolumn{1}{l}{$\delta x$} & \multicolumn{1}{l}{$\delta$} \\
\hline S1 & -0.0034 & 0.0000 & -0.0012 \\
S1_2 & -0.0098 & 0.0000 & -0.0035 \\
S1_3 & -0.0150 & 0.0000 & -0.0054 \\
S1_4 & -0.0196 & 0.0000 & -0.0070 \\
S1_5 & 0.0184 & 0.0000 & 0.0066 \\
C1 & -0.0012 & 0.0001 & -0.0026 \\
C1_2 & -0.0035 & -0.0001 & -0.0075 \\
C1_3 & -0.0054 & 0.0001 & -0.0114 \\
C1_4 & -0.0071 & 0.0000 & -0.0149 \\
C1_5 & 0.0066 & 0.0000 & 0.0140 \\
N1 & -0.0005 & 0.0002 & -0.0047 \\
N1_2 & -0.0010 & -0.0002 & -0.0107 \\
N1_3 & 0.0000 & 0.0002 & 0.0020 \\
N1_4 & -0.0013 & 0.0001 & -0.0155 \\
N1_5 & -0.0015 & 0.0000 & -0.0191 \\
N1_6 & -0.0010 & -0.0001 & -0.0138 \\
N1_7 & 0.0015 & -0.0001 & 0.0184 \\
N1_8 & 0.0013 & 0.0001 & 0.0175 \\
N1_9 & 0.0005 & 0.0002 & 0.0084 \\
\hline
\end{tabular}

the distortion mode it is necessary to give the mode displacements for all atoms in the large asymmetric unit of the distorted phase with 19 atoms. In these 19 atomic displacements the trivial correlations between consecutive parent unit cells along the modulation direction coming from the sinusoidal form of the mode/modulation are inextricably entangled with the non-trivial ones coming from the DT4 character of the corresponding irrep. In contrast, the definition of the first harmonic modulation in the superspace approach only requires giving explicitly the three amplitudes that determine the first harmonic of the modulation function for the three independent atoms in the asymmetric unit of the parent phase (Zuñiga et al., 1989). The sinusoidal function so defined, together with the modulation wavevector, is sufficient to describe the displacements of the equivalent atoms in the nine 


\section{Table 19}

Summary of the mode decomposition of the GaII phase with space group $C 222_{1}$ and 38 atomic positional parameters (Degtyareva et al., 2004) with respect to an $F d d d$ structure with a single independent atom at position $8 a(1 / 8,1 / 8,1 / 8)$.

The first three harmonics dominating the distortion can be identified.

\begin{tabular}{rlllll}
\hline $\begin{array}{l}\text { Order } \\
\text { harmonic }\end{array}$ & K-vector & Irrep & $\begin{array}{l}\text { Isotropy } \\
\text { subgroup }\end{array}$ & Dimension & $\begin{array}{l}\text { Amplitude } \\
(\AA)\end{array}$ \\
\hline 23 & $(0,0,1 / 13)$ & LD3 & $C 222_{1}$ & 2 & 0.11 \\
3 & $(0,0,1 / 13)$ & LD4 & $C 222_{1}$ & 2 & 2.14 \\
6 & $(0,0,2 / 13)$ & LD1 & $F 222$ & 1 & 0.03 \\
20 & $(0,0,2 / 13)$ & LD2 & $F d d d$ & 1 & 0.03 \\
17 & $(0,0,3 / 13)$ & LD3 & $C 222_{1}$ & 2 & 0.06 \\
9 & $(0,0,3 / 13)$ & LD4 & $C 222_{1}$ & 2 & 0.05 \\
12 & $(0,0,4 / 13)$ & LD1 & $F d d d$ & 1 & 0.00 \\
14 & $(0,0,4 / 13)$ & LD2 & $F 222$ & 1 & 0.03 \\
11 & $(0,0,5 / 13)$ & LD3 & $C 222_{1}$ & 2 & 0.05 \\
15 & $(0,0,5 / 13)$ & LD4 & $C 222_{1}$ & 2 & 0.11 \\
18 & $(0,0,6 / 13)$ & LD1 & $F 222$ & 1 & 0.06 \\
8 & $(0,0,6 / 13)$ & LD2 & $F d d d$ & 1 & 0.01 \\
5 & $(0,0,7 / 13)$ & LD3 & $C 222_{1}$ & 2 & 0.16 \\
21 & $(0,0,7 / 13)$ & LD4 & $C 222_{1}$ & 2 & 0.11 \\
24 & $(0,0,8 / 13)$ & LD1 & $F d d d$ & 1 & 0.03 \\
2 & $(0,0,8 / 13)$ & LD2 & $F 222$ & 1 & 0.53 \\
1 & $(0,0,9 / 13)$ & LD3 & $C 222_{1}$ & 2 & 3.59 \\
25 & $(0,0,9 / 13)$ & LD4 & $C 222_{1}$ & 2 & 0.05 \\
22 & $(0,0,10 / 13)$ & LD1 & $F 222$ & 1 & 0.02 \\
4 & $(0,0,10 / 13)$ & LD2 & $F d d d$ & 1 & 0.00 \\
7 & $(0,0,11 / 13)$ & LD3 & $C 222_{1}$ & 2 & 0.12 \\
19 & $(0,0,11 / 13)$ & LD4 & $C 222_{1}$ & 2 & 0.05 \\
16 & $(0,0,12 / 13)$ & LD1 & $F d d d$ & 1 & 0.04 \\
10 & $(0,0,12 / 13)$ & LD2 & $F 222$ & 1 & 0.01 \\
13 & $(0,0,1)$ & Z2 & $C 222_{1}$ & 2 & 0.07 \\
\hline
\end{tabular}

consecutive parent unit cells forming the superstructure unit cell, while the correlation according to irrep DT4 with those atoms that are symmetry related by rotational operations in the parent phase is automatically introduced by the superspace-group operations (Janssen et al., 2004; Perez-Mato et al., 1987).

As a final example we consider phase GaII of the element Ga under pressure, which to our knowledge is the most extreme case achieved in parameter reduction when describing a superstructure as a modulated structure within the superspace formalism. This phase of symmetry $C 222_{1}$ (Degtyareva et al., 2004), stable between 2 and $10 \mathrm{GPa}$, has a very large unit cell with 14 symmetry-independent Ga atoms, but it has been shown to be a simple commensurately modulated structure of an Fddd structure with only a single symmetry-independent atom at the Wyckoff position $8 a$ $(1 / 8,1 / 8,1 / 8)$ (Perez-Mato et al., 2006). The superspace group describing the symmetry properties of the modulation was found to be $F d d d(00 \gamma) 0 s 0$, with $\gamma=9 / 13$. Hence the modulation wavevector was chosen as (0 0 9/13) and the conventional unit cell is multiplied by 13 , with respect to the virtual Fddd parent structure. Within the superspace approach the number of possible harmonics for the single atomic modulation required to define the full structure is 25 . But the structure could be satisfactorily described with only three harmonics, which meant a reduction from 38 to four positional parameters, when passing from a conventional crystallographic description to a modulated one. The transformation relating the setting of the $F d d d$ parent structure with the experimental one (Degtyareva et al., 2004) can be chosen as $(\mathbf{a}, \mathbf{b}, 13 \mathbf{c} ;-1 / 8,1 / 8,-3 / 8)$. The maximum atomic displacement in the distortion relating both structures is of the order of $0.8 \AA$ and its mode decomposition is summarized in Table 19. The number of possible irrep distortions is 25 , in accordance with the number of allowed harmonics in the superspace description, but the irreps involved have only 13 possible wavevectors of type $(n / 13) c^{*}$, with two different possible small irreps for each of them except for $n=13$, which corresponds to the special point $\mathrm{Z}$ at the Brillouin-zone border and only one irrep is compatible. The irrep distortions with $n$ odd all have as isotropy subgroup the observed symmetry and are from this viewpoint possible primary modes, while the even modes are all secondary, with higher isotropy subgroups. The amplitudes obtained for the 25 symmetry components in the distortion shows the dominant role played by the LD3 distortion with wavevector $q=(9 / 13) c^{*}$, in accordance with the superspace description. This LD3(9/13) distortion is clearly the primary distortion, being more than one order of magnitude larger than the rest of the components, except for two additional irrep distortions, which can be identified with a second and a third harmonic. Indeed, the LD4[1/13] distortion with a considerable amplitude has a wavevector equivalent to $3 q$, while $\mathrm{LD} 2[8 / 13]$ can be identified with a second harmonic with wavevector $2 q$ equivalent to $-(8 / 13) c^{*}$. These three irrep distortions correspond therefore to the three first harmonics, which were considered sufficient by Perez-Mato et al. (2006) to describe the structure.

In general, for each wavevector $(n / 13) c^{*}$ two irrep distortions exist with symmetries LD3 and LD4 for odd terms and LD1 and LD2 for even terms, except for the case $n=13$, with a single irrep Z2. Each of these irrep distortions corresponds to one of the harmonics used in the superspace description. A modulation harmonic of order $m(m=2, \ldots, 25)$ in the superspace description is in fact a distortion with wavevector $q_{m}=(9 m / 13) c^{*}$ which can be changed to an equivalent wavevector $q_{n}=(n / 13) c^{*}(n=1, \ldots, 13)$ through a reciprocallattice translation $2 p c^{*}$ ( $p$ integer): $q_{n}=q_{m}+2 p c^{*}$ or $-q_{m}+$ $2 p c^{*}$. This change implies also changing the small irrep associated with the wavevector, depending on the parity of $p$, so that for $n$ odd it changes from LD3 ( $p$ even) to LD4 ( $p$ odd), while for $n$ even the change is from LD1 ( $p$ even) to LD2 ( $p$ odd). This property can be derived from the form of the irreps, and their labeling through a wavevector representative and a small irrep that depends on the chosen wavevector (Cracknell et al., 1979). Thus, for instance the LD3[1/13] distortion can be considered a 23rd harmonic, its wavevector being $q_{23}=$ $23(9 / 13) c^{*}=-(1 / 13) c^{*}+8\left(2 c^{*}\right)$ with $p$ even, while LD4[1/13] corresponds to the third-order harmonic with $q_{3}=3(9 / 13) c^{*}=$ $(1 / 13) c^{*}+2 c^{*}$, and $p$ odd. Following these rules the correspondence between the 25 harmonics of the superspace description and the 25 irrep components of the mode decomposition can be made. This is given in the first column of Table 19. The equivalence between the two approaches can be clearly seen, and also that the superspace approach can deal 
with the symmetry constraints present in each harmonic in a more efficient way.

The choice of the superspace group implies a decision about which of the 25 irrep distortions is the primary modulation. Once a superspace group is assumed, the superspace symmetry automatically introduces the symmetry properties of each harmonic, and a rough hierarchy in their importance is implicitly assumed. The mode decomposition, on the other hand, does not assume a priori any predominance among the 25 irrep components, and one has to define and indicate explicitly one by one the symmetry properties of each possible irrep distortion present in the structure. Only the actual mode decomposition and the values of the distortion amplitudes for a given structure will allow one to identify whether a primary prevailing distortion exists. Even if a primary distortion has been $a$ priori identified from experimental results, the identification of the secondary modes of lowest order among all possible irrep distortions, susceptible of having more weight in the total distortion, require non-trivial considerations about the relation between irrep labeling and wavevector choice, as shown above.

Although the structure of GaII, as stressed by Perez-Mato et al. (2006), is clearly very well described by the three first modulation harmonics, indicated in Table 19, there are some additional distortion amplitudes which seem to be significant (being clearly larger than their standard deviations). It is remarkable that even for these smaller components the underlying hierarchy for odd harmonics coming from the primary mode is observed, a fact that supports the consistency of these smaller components of the reported structural model. Thus, as shown in Table 19, the next two irrep distortions with the largest amplitudes of 0.16 and $0.12 \AA$ can be identified with the fifth and seventh modulation harmonic. On the other hand, the secondary modes with $n$ even, except the second harmonic, have amplitudes that can be taken as zero, considering their standard deviation.

Summarizing, one can say that the use of symmetry modes in the description of distorted structures is quite similar to the application of superspace symmetry. Both approaches allow in commensurate distorted structures a division of the configuration space into symmetry-adapted subspaces, which have usually very different weights in the total distortion. However, the superspace symmetry of a commensurate structure is not unique, and in this case the choice of the superspace group is equivalent to a choice (among the possible ones) of the irrep mode(s) that have a primary role and prevail in the distortion. In contrast, a parameterization of the structure in terms of symmetry modes does not require introducing a priori such hierarchy among the irrep components. On the other hand, the superspace formalism introduces a much more efficient and economical form of describing the irrep distortions, if these are modulations with long wavelengths compared with the size of the parent unit cell. Reversely, for superstructures with a small cell multiplication, including cases with several independent wavevectors, the symmetry-mode decomposition is more efficient and simpler than the superspace method.
The choice of the most adequate superspace group for a commensurate structure can in some cases be problematic or ambiguous. In these cases the symmetry-mode approach can help to make the right choice of the superspace group by identifying one or several primary active irreps. It is in general possible to derive the resulting possible superspace groups from an active irrep (Perez-Mato et al., 1984; Stokes et al., 2007).

\section{Mode analysis in ab initio calculations: a natural basis of symmetry modes}

\subsection{Constraining the configuration space}

Quantum $a b$ initio calculations within the formalism of the DFT are becoming rather common tools for explaining the stability of distorted phases and for predicting their structures (Rabe et al., 2007; Yin \& Cohen, 1982; Milman et al., 2000). These calculations permit one in general to determine within a good approximation the energy and atomic forces for any crystalline configuration. By this means, structures can be relaxed in the computer and a prediction for the ground state of the system can be obtained. Despite complex extensions and improvements of this basic relaxation process (Oganov \& Glass, 2006), these $a b$ initio calculations are limited by the fact that the configuration space being explored must always be constrained to a small subspace. The choice of this restricted subspace is a rather empirical matter, despite the vocation of these calculations being independent of experimental knowledge.

Another limitation is the fact that these methods only calculate properties at $0 \mathrm{~K}$, and therefore are only valid for determining the ground-state configuration and not phases that are stabilized by thermal effects. Some thermal effects can be calculated with more computer-costly ab initio molecular dynamics (Car \& Parrinello, 1985), or with Monte Carlo calculations introducing further approximations as effective Hamiltonians (Zhong et al., 1995). But, also within these techniques, the empirical constraint on the explored configuration space plays a fundamental role and can be critical in obtaining correct predictions.

The reduction of the explored configuration space is often carried out by considering a limited set of possible space groups (subgroups of the parent space group). From what has been shown in the sections above, it is clear that symmetrymode analysis can be a tool of extraordinary efficiency for identifying the relevant symmetries that are worth exploring. The distorted perovskites discussed above are a clear example. Most of these phases are caused by one or several symmetry modes, which tend to be systematically unstable for many compositions. Distortions corresponding to the irreps $\mathrm{R} 4+$, M3+ and GM4- can be found acting as primary irrep distortions in many of these phases, yielding quite a various but limited set of space-group symmetries. These possible space groups depend on the direction taken by each distortion mode within its irrep space, and the actual combination of the three irreps realized in each case. 


\section{Table 20}

Isotropy subgroups of $P m \overline{3} m$ corresponding to the presence of distortions of symmetry GM4- and R4+, as obtained using ISOTROPY (Stokes \& Hatch, 2002), relevant for considering possible symmetries of phases of $\mathrm{BiFeO}_{3}$.

The first two columns show the special direction (one of a set of equivalent ones) within the irrep space corresponding to the listed subgroup. The conventional unit cell and the origin shift of the subgroup is indicated in parentheses for each subgroup. First the possible symmetries when only one of the two irrep distortions are listed. Then the possible subgroups for distortions including the two irreps are shown.

\begin{tabular}{|c|c|c|}
\hline $\begin{array}{l}\text { Distortion } \\
\text { GM4- }\end{array}$ & $\begin{array}{l}\text { Distortion } \\
\text { R4+ }\end{array}$ & Subgroup \\
\hline$(a, 0,0)$ & - & $P 4 m m(\mathbf{b}, \mathbf{c}, \mathbf{a} ; 0,0,0)$ \\
\hline$(a, a, 0)$ & - & $A m m 2(\mathbf{c}, \mathbf{a}-\mathbf{b}, \mathbf{a}+\mathbf{b} ; 0,0,0)$ \\
\hline$(a, a, a)$ & - & $R 3 m(\mathbf{a}-\mathbf{b}, \mathbf{b}-\mathbf{c}, \mathbf{a}+\mathbf{b}+\mathbf{c} ; 0,0,0)$ \\
\hline$(a, b, 0)$ & - & $P m(\mathbf{b}, \mathbf{c}, \mathbf{a} ; 0,0,0)$ \\
\hline$(a, a, b)$ & - & $C m(\mathbf{a}+\mathbf{b}, \mathbf{b}-\mathbf{a}, \mathbf{c} ; 0,0,0)$ \\
\hline$(a, b, c)$ & - & $P 1(\mathbf{a}, \mathbf{b}, \mathbf{c} ; 0,0,0)$ \\
\hline - & $(a, 0,0)$ & $I 4 / \mathrm{mcm}(\mathbf{a}+\mathbf{b}, \mathbf{b}-\mathbf{a}, 2 \mathbf{c} ; 0,0,0)$ \\
\hline- & $(a, a, 0)$ & $\operatorname{Imma}(\mathbf{a}+\mathbf{c}, 2 \mathbf{b}, \mathbf{c}-\mathbf{a} ; 0,0,0)$ \\
\hline - & $(a, a, a)$ & $R \overline{3} c(\mathbf{b}-\mathbf{a}, \mathbf{c}-\mathbf{b}, 2 \mathbf{a}+2 \mathbf{b}+2 \mathbf{c} ; 0,0,0)$ \\
\hline - & $(a, b, 0)$ & $C 2 / m(-2 \mathbf{c}, 2 \mathbf{b}, \mathbf{a}+\mathbf{c} ; 0,1 / 2,1 / 2)$ \\
\hline- & $(a, a, b)$ & $C 2 / c(-\mathbf{a}+2 \mathbf{b}-\mathbf{c}, \mathbf{c}-\mathbf{a}, \mathbf{a}+\mathbf{c} ; 0,1 / 2,1 / 2)$ \\
\hline - & $(a, b, c)$ & $P \overline{1}(\mathbf{b}+\mathbf{c}, \mathbf{a}+\mathbf{c}, \mathbf{a}+\mathbf{b} ; 0,0,0)$ \\
\hline$(a, a, a)$ & $(b, b, b)$ & $R 3 c(\mathbf{b}-\mathbf{a}, \mathbf{c}-\mathbf{b}, 2 \mathbf{a}+2 \mathbf{b}+2 \mathbf{c} ; 0,0,0)$ \\
\hline$(a, 0,0)$ & $(b, 0,0)$ & $\operatorname{Fmm} 2(2 \mathbf{c}, 2 \mathbf{b},-2 \mathbf{a} ;-1 / 2,1 / 2,0)$ \\
\hline$(a, 0,0)$ & $(0, b, 0)$ & $I 4 \mathrm{~cm}(\mathbf{b}+\mathbf{c}, \mathbf{c}-\mathbf{b}, 2 \mathbf{a} ; 0,0,0)$ \\
\hline$(a, a, 0)$ & $(b, 0,0)$ & $\operatorname{Ima2}(2 \mathbf{c}, \mathbf{b}-\mathbf{a},-\mathbf{a}-\mathbf{b} ;-3 / 4,1 / 4,-1 / 2)$ \\
\hline$(a, b, 0)$ & $(c, 0,0)$ & $C m(-2 \mathbf{a},-2 \mathbf{c}, \mathbf{a}-\mathbf{b} ; 0,0,0)$ \\
\hline$(a, 0,0)$ & $(b, 0, b)$ & $\operatorname{Ima2}(\mathbf{b}+\mathbf{c}, \mathbf{b}-\mathbf{c}-2 \mathbf{a} ;-1 / 2,0,1 / 2)$ \\
\hline$(a, a, 0)$ & $(0, b, b)$ & $\operatorname{Ima} 2(2 \mathbf{c}, \mathbf{b}-\mathbf{a},-\mathbf{a}-\mathbf{b} ; 0,0,0)$ \\
\hline$(a, a, 0)$ & $(0, b,-b)$ & $\operatorname{Imm} 2(\mathbf{a}-\mathbf{b}, 2 \mathbf{c},-\mathbf{a}-\mathbf{b} ; 0,0,1 / 2)$ \\
\hline$(a, a, b)$ & $(0, c,-c)$ & $\mathrm{Cm}(-\mathbf{a}-\mathbf{b}-2 \mathbf{c}, \mathbf{b}-\mathbf{a}, \mathbf{a}+\mathbf{b} ; 0,0,0)$ \\
\hline$(a, a, 0)$ & $(-c, b,-b)$ & $C 2(\mathbf{b}-\mathbf{a}-2 \mathbf{c}, \mathbf{a}+\mathbf{b}, \mathbf{a}-\mathbf{b}, 1 / 4,1 / 4,-1 / 2)$ \\
\hline$(a, a, b)$ & $(d, c, c)$ & $C c(-\mathbf{a}-\mathbf{b}-2 \mathbf{c}, \mathbf{b}-\mathbf{a}, \mathbf{a}+\mathbf{b} ;-1 / 2,0,-1 / 2)$ \\
\hline$(a, 0,0)$ & $(c, 0, b)$ & $C 2(-2 \mathbf{b}, 2 \mathbf{a}, \mathbf{b}+\mathbf{c} ; 1 / 2,-1 / 2,0)$ \\
\hline$(a, b, 0)$ & $(0, c, d)$ & $\mathrm{Cm}(-2 \mathbf{a},-2 \mathbf{c}, \mathbf{a}-\mathbf{b} ;-1 / 2,01 / 2)$ \\
\hline$(a, b, c)$ & $(d, e, f)$ & $P 1(-\mathbf{a}-\mathbf{c}, \mathbf{a}+\mathbf{b}, \mathbf{a}-\mathbf{b} ; 0,0,0)$ \\
\hline
\end{tabular}

Let us consider, for instance, the case of multiferroic $\mathrm{BiFeO}_{3}$. Despite the intensive research on this material, its thermal phase diagram is still under discussion. Its roomtemperature phase has $R 3 c$ symmetry, and can be decomposed into R4+ and GM4- contributions. If we are interested in determining other phases of the phase diagram, it is then reasonable to expect that these phases correspond to other possible combinations of R4+ and GM4- distortions, or are due to only one of these two irrep distortions. Using freely available tools such as ISODISPLACE (Campbell et al., 2006), one can find automatically all possible space groups that can result from the action of these two irreps as primary distortions, acting together or in isolation. These symmetries are shown in Table 20 and are the obvious candidates for any intermediate or competing phase. Furthermore, if we are trying to characterize an intermediate paraelectric phase realized before a full symmetrization into the parent cubic symmetry (the so-called $\beta$ phase), then the obvious symmetry choices are the isotropy subgroups corresponding to a single R4+ distortion, also listed Table 20. Six different space groups are then possible. Only a couple of these symmetries have appeared in the considerations of some of the published $a b$ initio or experimental studies of $\mathrm{BiFeO}_{3}$ (Haumont et al., 2008; Kornev \& Bellaiche, 2009; Kornev et al., 2007) and the symmetry of this intermediate phase is still a matter of discussion (Catalan \& Scott, 2009; Selbach et al., 2008). Unfortunately, a systematic exploration, either experimentally or with ab initio techniques consistent with the mode analysis, taking into account these six possibilities, has not been made.

Some recent work has reported that this $\beta$ phase of $\mathrm{BiFeO}_{3}$ has Pbnm symmetry (conventional Pnma) (Arnold et al., 2009). As can be seen in Table 20, this symmetry cannot be explained by the action of a R4+ distortion or the combination of R4+ and GM4-. This phase would be the Pnma configuration, typical of many perovskites, that was discussed in $\S 9$. As shown there, it requires, besides a R4+ distortion, an M3+ distortion as primary agent of the symmetry break. This Pnma phase would then imply that not only the polar distortion GM4- disappears at the ferroelectric-paraelectric transition but also an additional M3+ distortion simultaneously condenses. This is in principle possible, as the transition is strongly first order. But a full validation of the model, given the uncertainties of powder diffraction analysis of pseudosymmetric structures, would require exploring and checking first the simpler scenarios represented by the action of only a R4+ distortion, whose possible space groups are listed in Table 20. The need for defining a limited set of possible reasonable alternative symmetry scenarios is even more peremptory if we would pretend to predict or determine these phases by ab initio methods. As seen in this example, the analysis of the known structures in terms of irrep distortions can be a most valuable tool for this task.

\subsection{Comparison of ab initio structures with experimental ones}

$A b$ initio calculations are frequently used to determine/ predict the structure corresponding to the ground state of the studied compound. As stressed above, the calculation is in general limited by the assumed symmetry of this state. Some good approximations to the structures of thermally stabilized phases can also be obtained by restricting the minimization of the energy within a postulated or an experimentally known space-group symmetry. The comparison of these theoretical structural models with experimental ones is usually made at a qualitative level, comparing one-to-one the atomic positions. We have seen in the previous sections that the decomposition in terms of symmetry modes provides a robust framework for making quantitative comparisons of experimental structures having equal or different symmetry. As shown in $\S 8$, the separation in a given structural distortion of the irrep distortion amplitudes and the corresponding normalized polarization vectors, together with the distinction between primary and secondary irrep distortions, permits quantitative comparisons of different structural models with deeper insight, separating marginal from fundamental structural features. This is also valid for the comparison of ab initio calculated relaxed structures with experimental ones. The athermal character of the $a b$ initio calculations is bound to yield distortion amplitudes in general larger than those observed in experiment, while the polarization vectors of the 
irrep distortions are expected to be rather robust, weakly dependent on temperature and therefore well described by the calculated models. By means of the symmetry-mode decomposition, the extreme quantitative agreement of sound $a b$ initio calculations with experimental structural features can become patent.

As an example, let us consider the case of $\mathrm{Bi}_{4} \mathrm{Ti}_{3} \mathrm{O}_{12}$. This Aurivillius compound has a tetragonal $14 / \mathrm{mmm}$ configuration as parent structure, and its room-temperature ferroelectric phase was the subject of some controversy. While powder $\mathrm{X}$-ray and neutron powder diffraction studies (Zhou et al., 2003; Hervoches \& Lightfoot, 1999) reported a structure with space group $B 2 c b$ [No. 41, B2eb in the new standard notation (Hahn, 2002)], which is the usual symmetry within the family, an early single-crystal X-ray analysis (Rae et al., 1990) claimed that the phase was monoclinic, with space group $B 1 a 1$, a subgroup of $B 2 c b$. The monoclinic angle was $90^{\circ}$ within experimental resolution, making difficult the experimental detection of this symmetry reduction with powder diffraction experiments. However, a first-principles relaxation of the structure within the discussed $B 1 a 1$ symmetry and its comparison with the experimental monoclinic structure, decomposed into irrep distortions, dissipates any possible doubt about the actual symmetry of this phase (Perez-Mato $e t$ $a l ., 2008)$. The $B 1 a 1$ structure has 57 free positional atomic parameters, compared with six in the parent tetragonal structure. The additional 51 degrees of freedom can be divided into seven distinct irrep subspaces. For our purposes the specific labels and symmetry-breaking properties of these seven irreps are not important [more details can be seen in Perez-Mato et al. (2008)], except that three of them are compatible with the space group $B 2 c b$ and the remaining four are added by the reduction of the symmetry to $B 1 a 1$. Ordering these seven subspaces in a fixed way, with the three irreps consistent with the $B 2 c b$ symmetry first, the experimental monoclinic structure can be described by a vector with seven components/amplitudes (in $\AA$ ), namely $(1.60,1.43,0.96,0.62$, $0.08,0.22,0.13)$, and seven specific normalized polarized vectors defined in each subspace. The dimensions of the seven subspaces are $8,11,3,5,8,9$ and 7 , respectively. One can see in the seven-component vector the prevailing weight of the first three distortion components, i.e. those that are consistent with the $B 2 c b$ space group. The modulus of the seven-component vector, i.e. the total amplitude of the structural distortion, is $2.45 \AA$, while the three first irrep components already constitute a distortion of $2.35 \AA$. The remaining orthogonal distortion that reduces the symmetry to $B 1 a 1$ has a total amplitude of $0.68 \AA$. This latter is therefore much weaker, but certainly significant, with a clear predominance of the first of the four additional irrep components with an amplitude of $0.62 \AA$. The computer-calculated relaxation of the structure within the $B 1 a 1$ symmetry resulted in a structure described using the same parameterization by the following vector: $(1.77,1.33$, $1.07,0.82,0.10,0.25,0.11)$. As expected, the amplitudes are in general slightly larger: $2.61 \AA$ versus $2.45 \AA$ for the total amplitude, $2.46 \AA$ versus $2.35 \AA$ for the $B 2 c b$ distortion (first three components) and $0.87 \AA$ versus $0.68 \AA$ for the modulus of the remaining four components. This slight increase is quite homogeneous for the seven amplitudes, the relative values following the same pattern as in the experimental structure. This similitude of the two structural models can be quantified by the parallelism of the directions defined by the two vectors above; their scalar product is 0.995 , very close to the ideal parallelism.

One can further compare the internal form of each irrep distortion component through the values of the scalar products of their normalized polarization vectors derived from the two structural models. These scalar products result to be $-0.998,-0.996,0.998,0.99,-0.73,-0.80$ and 0.64 , respectively. This means that a more adequate description of the calculated structure, defining polarization vectors similar to those used for the description of the experimental structure, would require changing the signs of some amplitudes: $(-1.77$, $-1.33,1.07,0.82,-0.10,-0.25,0.11)$. One can check that these correlated changes of sign of some of the irrep distortion amplitudes correspond to an equivalent domain-related configuration, as discussed in $\S 7$, and are therefore not important. Note, however, that they imply that a conventional mapping of the two structures comparing one-to-one the atomic positions makes no sense in general. In contrast, the detailed comparison in terms of symmetry modes demonstrates quantitatively the striking agreement of the two structural models, once the expected larger amplitude of the theoretical distortion is taken into account. The dominant irrep components in the two structures agree perfectly, as shown by their scalar products; in particular, the irrep distortion defined by five parameters with amplitude $0.62 \AA$, which is mainly responsible for the symmetry reduction to $B 1 a 1$, is reproduced by the theoretical calculation up to a coincidence given by a scalar product of 0.99 . One can surely not only take for granted that the compound is monoclinic, but also confirm the monoclinic structural model. Note, however, how the agreement of the weaker secondary components is significantly worse. For the irrep components with amplitudes of the order of $0.1 \AA$, the scalar product of their polarization components reduces to values of the order of 0.7. Although the polarization vectors of weaker distortions are necessarily less accurate (the error increasing as the irrep distortion amplitude decreases), the deterioration of the agreement is somewhat larger than expected from this effect. It seems that weaker marginal components are less well determined, as happens in the comparison of experimental structures.

The high level of agreement between $a b$ initio and experimental structures shown in the example above is not exceptional when given in this detailed quantitative form. In PerezMato et al. (2009) the $P 2_{1}$ experimental structure of $\mathrm{SrAl}_{2} \mathrm{O}_{4}$ was compared with an $a b$ initio relaxed structure and the coincidence of both models is also excellent. This structure can be considered a distortion from a $P 6_{3} 22$ parent phase. The system has five irrep distortions; their symmetry labels and respective isotropy subgroups are indicated in Fig. 16. Ordering the components in the form (M2-1q, GM6, M3-1q, GM5, GM4), the vectors describing the amplitudes of the irrep distortion components were $(1.70,1.39,0.57,0.32,0.02)$ and 
$(1.81,1.35,0.57,0.24,0.03)$ for the experimental and the ab initio calculated structure, respectively, with their directions having 0.998 as scalar product. In addition, the scalar products of the polarization vectors of the five irrep distortions in the two structures were $0.998,0.9997,0.997,0.96$ and 0.63 . We can compare in more detail the polarization vectors of the primary distortion having $1.70 \AA$ and $1.81 \AA$ amplitudes in the experimental and theoretical structures, labeled as M2-1q. Using a set of 12 basis modes, the experimental polarization vector for this distortion is $(-0.15,0.10,-0.17,-0.22,-0.14$, $-0.11,-0.69,-0.53,0.06,-0.14,0.05,-0.31)$, while that in the $a b$ initio relaxed structure is $(-0.14,0.07,-0.15,-0.22,-0.13$, $-0.10,-0.69,-0.54,0.05,-0.11,0.02,-0.31)$. Note that owing to the different total amplitude of the distortion in the experimental and theoretical structures, and the contribution of the weaker irrep distortions, this almost perfect agreement on this primary distortion could not be detected by a direct comparison of the atomic coordinates.

\subsection{Primary and secondary distortions in ab initio calculations}

The different roles played by primary and secondary distortions in the stabilization of distorted phases can be investigated by means of $a b$ initio calculations of the energy landscape as a function of the amplitudes of the different irrep distortions detected in the experimental or in the $a b$ initio relaxed structure. We have seen that the symmetry-mode decomposition permits one in general to distinguish the collective degrees of freedom that are unstable in the parent phase and are responsible for the existence of the observed phase from those that are present in the distortion, but are irrelevant for the transition mechanism, in the sense that the transition would take place independently of their presence or not. These modes are in fact stable or 'hard' modes, despite

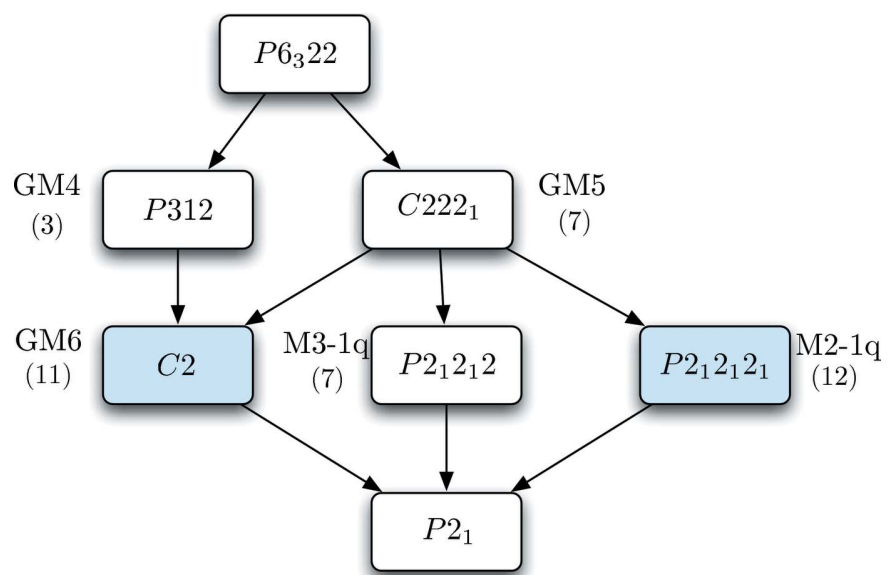

Figure 16

Graph of maximal subgroups relating the space groups of the parent and distorted phase of $\mathrm{SrAl}_{2} \mathrm{O}_{4}$. For each subgroup any irrep yielding this symmetry is indicated. The two subgroups corresponding to the primary active irreps evidenced by the mode decomposition are highlighted. The dimension of the configuration subspace corresponding to each irrep distortion is indicated in parentheses. their condensation through coupling with the primary distortions.

As an example, Fig. 17 shows the energy variation in $\mathrm{SrAl}_{2} \mathrm{O}_{4}$ (Perez-Mato et al., 2009) as a function of the amplitude of the primary distortion GM6 observed in the experimental $P 2_{1}$ structure, the one with amplitude $1.39 \AA$ (see above). This energy variation is compared with that resulting from the secondary distortion M3-1q also present in the experimental structure with an amplitude of $0.57 \AA$. A schematic view of these distortions is given in Fig. 18. While the polar GM6 distortion corresponds to an instability of the parent configuration, the M3-1q distortion that has a weaker but significant weight in the distortion is a 'hard mode'. As shown in Fig. 17, this distortion component by itself is energetically unfavorable, and, if it could be hindered, the structural instability of the parent structure would still persist.

The expected very different response to external perturbations of the irrep distortions becomes evident when the resulting $a b$ initio structures are analyzed in terms of symmetry modes. Fig. 19 shows the amplitudes of the two primary unstable distortions, GM6 and M2-1q, present in the $a b$ initio calculated structures as the monoclinic $\beta$ angle is varied parametrically, its equilibrium value being about $93.5^{\circ}$ (or $86.5^{\circ}$ ). In this compound the monoclinic strain is bilinearly coupled with the irrep distortion GM6, while only higherorder coupling terms, biquadratic or higher, are allowed with the second primary distortion M2-1q. This means that the GM6 distortion is expected to respond linearly to changes in the monoclinic shear strain, while the response of the M2-1q would be much weaker and not linear. Furthermore, the change of sign of the monoclinic shear strain should imply a corresponding switch of the GM6 distortion and the associated dielectric polarization, making the system transit into an equivalent domain-related configuration, with the GM6 distortion having an opposite amplitude, while the M2-1q distortion is maintained, in accordance with the general rules discussed in $\$ 7$. Fig. 19 shows that indeed the responses of the two irrep distortions to the forced shear strain follow this expected pattern. In fact, the M2-1q distortion is essentially

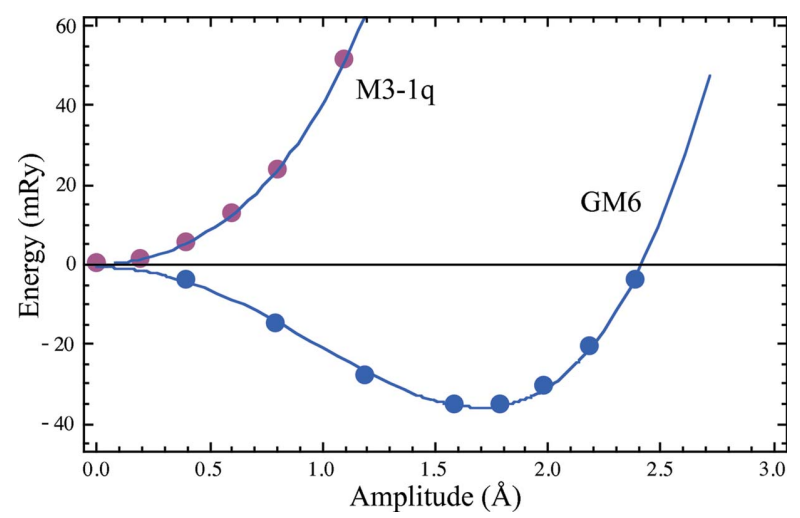

Figure 17

Calculated relative variation of the energy per formula unit in $\mathrm{SrAl}_{2} \mathrm{O}_{4}$ as a function of either the amplitude of the GM6 distortion or the amplitude of the M3-1q distortion, both present in the $P 2_{1}$ structure of this system. 
insensitive to the change of the monoclinic angle, while the GM6 distortion shows the expected switch and linear variation. Furthermore, the variation of the internal structure of the two distortions, monitored by the scalar product of their polarization vectors with those for a reference value of the monoclinic angle, is minimal, demonstrating that in a first approximation the variation of the structure is reduced to changes of some of the irrep distortion amplitudes. On the other hand, the variation of the polarization vector of the M3-1q distortion is significantly larger. As expected, this secondary distortion, being a higher-order coupling effect, with contributions of similar weight of different hard modes, suffers a stronger variation in its internal structure.

\subsection{The privileged basis of energy eigenmodes}

In $\$ 4$ we introduced the special physically adapted basis of symmetry modes $\left\{\mathbf{e}_{\mathrm{E}}(\tau, n)\right\}$ constituted by the eigenmodes of the matrix of second derivatives of the free energy for the atomic displacements with respect to the parent configuration. Quantum $a b$ initio calculations within the DFT formalism allow a theoretical determination of this privileged basis of eigenmodes, at least in the athermal approximation (i.e. assuming $0 \mathrm{~K})$. Branches of stiffness constants $\kappa_{\tau, n}(\mathbf{k})$ within the Brillouin zone, analogous to phonon branches, can be

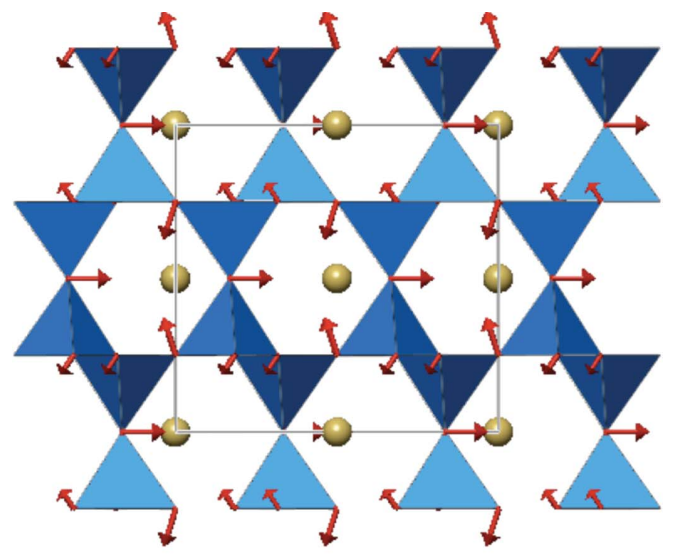

(a)

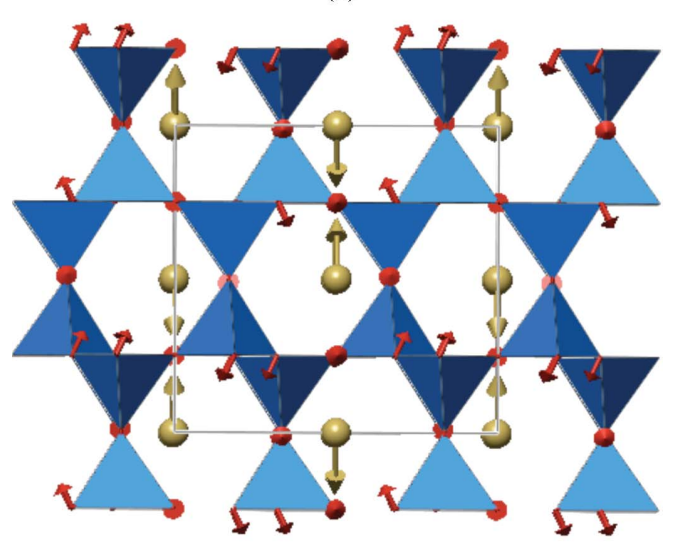

(b)

Figure 18

Scheme of the polarization vectors of $(a)$ the GM6 distortion and $(b)$ the M3-1q distortion present in the $P 2_{1}$ structure of $\mathrm{SrAl}_{2} \mathrm{O}_{4}$. calculated. The more unstable modes, i.e. those with most negative and lowest stiffness constants, are expected to prevail in the distorted phase (or phases) as primary modes. This is the basis for the construction of effective Hamiltonians for thermal simulations, where only the degrees of freedom corresponding to the lowest stiffness constant branches are included, the rest being subsumed within the heat bath. This approach implies two important approximations:

(i) neglecting the secondary (hard) modes;

(ii) the frozen primary distortions are supposed to correspond to the static eigenmodes of appropriate symmetry with the lowest stiffness coefficient.

Comparing the symmetry-mode decomposition of experimental or ab initio relaxed structures with the calculated basis of energy eigenmodes one can obtain an assessment of the validity of these approximations. In previous sections we have seen examples where secondary distortions of different symmetry than the primary distortion(s) are indeed quite negligible, as in $\mathrm{BaTiO}_{3}$, but we have also shown other cases where, although they have much smaller amplitudes, the secondary distortions are clearly significant. One cannot quantify their importance in the thermal properties from their

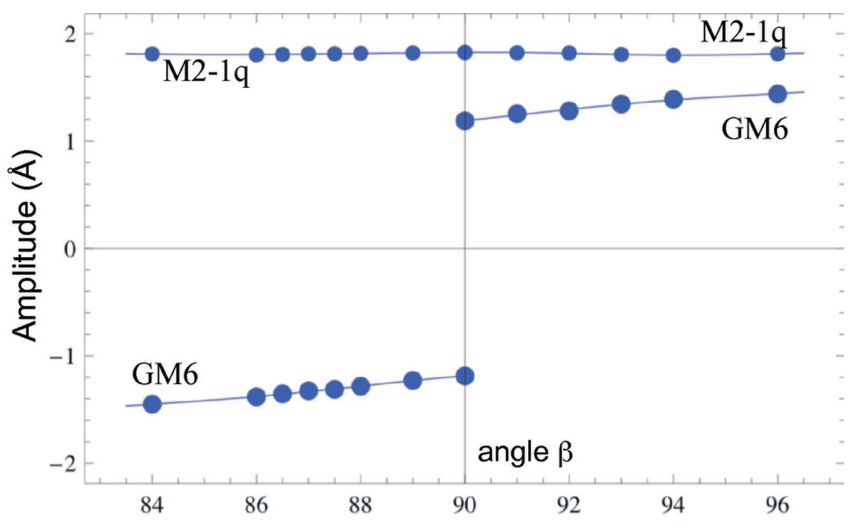

(a)

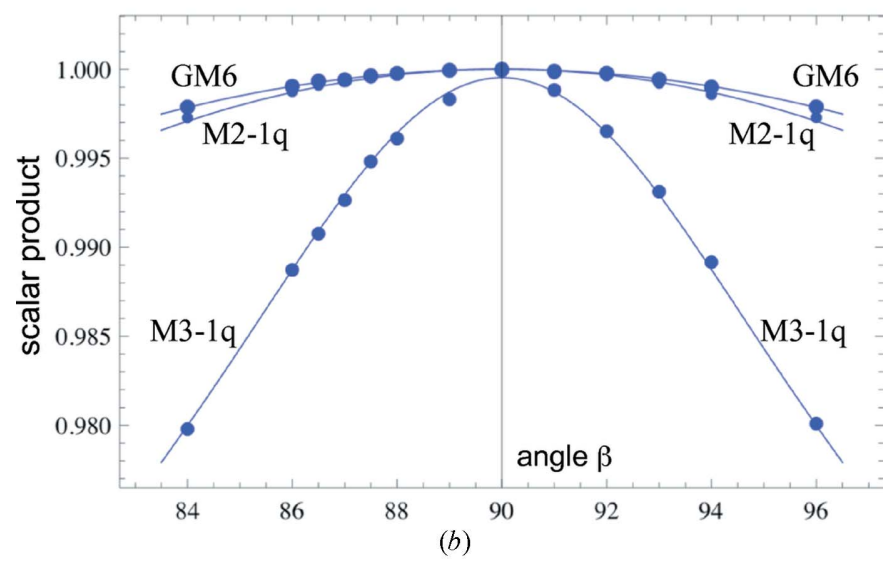

Figure 19

Calculated variation of some of the irrep distortions present in the $P 2_{1}$ structure of $\mathrm{SrAl}_{2} \mathrm{O}_{4}$ as a function of the angle $\beta$ of the monoclinic cell. (a) Amplitudes of the primary GM6 and M2-1q distortions. (b) Scalar product of the polarization vectors of the calculated GM6, M2-1q and M3-1q distortions with those corresponding to $\beta=90^{\circ}$. 
weight in the frozen static distortion, but one can expect a rough direct proportion between both.

Although hard secondary modes are irrelevant for the existence of the associated instabilities, they can play a fundamental role in the stabilization of the primary mode(s) along a specific direction within the irrep subspace, and therefore in the understanding of the phase diagram and the symmetries realized in the different phases. The identification of these significant secondary irrep distortions by means of a symmetry-mode decomposition of the experimental or the $a b$ initio relaxed structure is then the first step for improving the effective Hamiltonian method by including these additional degrees of freedom.

The validity of the second approximation within the effective Hamiltonian method mentioned above can be checked using the basis of calculated energy eigenmodes to describe the observed primary distortions. In the examples above, the polarization vectors of the irrep distortions were expressed in an arbitrarily chosen basis of symmetry-adapted modes. Once the static eigenmodes $\left\{\mathbf{e}_{\mathrm{E}}(\tau, n)\right\}$ for a certain irrep $\tau$ are calculated, the amplitudes $a_{\tau, n}^{\mathrm{E}}$ in equation (11), describing the observed irrep distortion $\mathbf{e}(\tau)$ in the basis of energy eigenmodes, can be immediately obtained through the scalar products $\mathbf{e}_{\mathrm{E}}(\tau, n) \cdot \mathbf{e}(\tau)$.

Let us consider the $\mathrm{X} 3$ - primary distortion (with respect to a tetragonal $I 4 / \mathrm{mmm}$ parent configuration) present in the orthorhombic phase of $\mathrm{SrBi}_{2} \mathrm{Ta}_{2} \mathrm{O}_{9}$ (Perez-Mato et al., 2004; Orobengoa et al., 2009). This distortion was shown to be described by the vector $(0.94,0.34,0.06,0.06,0.01,0.03,0.01)$ in the basis of energy X3 - eigenmodes of the parent structure, ordered from the lowest to the largest stiffness constant. In this basis, only the first eigenmode is unstable, the other six all having positive stiffness constants (Perez-Mato et al., 2004). One can see that indeed the unstable mode is dominant in the condensed distortion, but hard X3- eigenmodes have also small but significant contributions, which, as expected, decrease in general with the hardness of the eigenmode (see §). In particular, the second eigenmode, which is the softest one after the unstable one, but nevertheless stable, has a considerable weight in the static X3- distortion. Similarly, a second primary distortion of symmetry GM5 - of polar character, responsible for the spontaneous polarization of this compound, was given on the basis of calculated GM5eigenmodes by the vector $(0.88,0.32,0.14,0.07,0.09,0.06$, $0.28)$ with the components also ordered from softer to harder eigenmodes (Perez-Mato et al., 2004). Also here, only the first eigenmode is unstable, and one can see an important contribution of the second mode, despite being stable. Furthermore, in this case, it is remarkable that the hardest eigenmode, which corresponds to an internal vibrational mode of the $\mathrm{TaO}_{6}$ octahedra, has a large weight in the distortion, breaking the general trend of decreasing amplitudes with the hardness of the eigenmode. This is due to a very strong anharmonic coupling of this mode with the unstable first eigenmode, dominant in the frozen GM5- distortion.

This mode analysis of the experimental structure, combined with ab initio calculations, shows that hard eigenmodes with the same symmetry as the unstable ones can participate significantly in the distorted phase. This effect can also be observed in the distortions of simple ferroelectric perovskites. These hard eigenmodes are, however, excluded from the restricted configuration subspace usually considered in the construction of $a b$ initio effective Hamiltonians. This is probably one of the major sources of error of the method.

\section{Conclusions}

The development of programs such as AMPLIMODES (Orobengoa et al. 2009) and ISODISPLACE (Campbell et al., 2006) and their direct coupling with refinement programs now allow one to approach without much effort the structural characterization of any ordered pseudosymmetric structure with the full power of group representation theory. The decomposition of displacive distorted structures into symmetry-adapted modes can be performed in a straightforward manner, and permits strong fundamental distortions to be distinguished from those having a marginal character. A natural hierarchy among the different structural symmetry components exists in general, and is one of the fundamental advantages of this approach. The parameterization of the structures in terms of symmetry modes becomes closer to the physico-chemical mechanisms responsible for their stability, and is particularly appropriate for comparative studies. This paper has been devoted to demonstrating through various examples the power of this mode crystallography when applied a posteriori to a known structure, but it is obvious that its advantages are extensible to its direct use in the determination of the structures, refining directly the collective symmetry-mode coordinates. This is today within easy reach of anyone using the mentioned programs.

This work has been supported by the Spanish Ministry of Science and Innovation (project MAT2008-05839) and the Basque Government (project IT-282-07). The authors gratefully acknowledge most valuable discussions with H. Stokes, B. Campbell, Juan Rodríguez-Carvajal and R. L. Withers. We specially thank B. Kennedy and C. Howard for making available to us very useful unpublished data on $\mathrm{SrZrO}_{3}$.

\section{References}

Aizu, K. (1974). J. Phys. Soc. Jpn, 37, 885-896.

Aramburu, I., Friese, K., Perez-Mato, J. M., Morgenroth, W., Aroyo, M. I., Breczewski, T. \& Madariaga, G. (2006). Phys. Rev. B, 73, 014112.

Arnold, D. C., Knight, K. S., Morrison, F. D. \& Lightfoot, P. (2009). Phys. Rev. Lett. 102, 027602.

Aroyo, M. I., Kirov, A., Capillas, C., Perez-Mato, J. M. \& Wondratschek, H. (2006). Acta Cryst. A62, 115-128.

Aroyo, M. I., Perez-Mato, J. M., Capillas, C., Kroumova, E., Ivantchev, S., Madariaga, G. \& Kirov, A. (2006). Z. Kristallogr. 221, 15-27.

Authier, A. (2003). International Tables for Crystallography, Volume D, ch. 1.1. Dordrecht: Kluwer.

Avdeev, M., Caspi, E. N. \& Yakovlev, S. (2007). Acta Cryst. B63, $363-$ 372.

Bergerhoff, G. \& Brown, I. D. (1987). Crystallographic Databases. Chester: International Union of Crystallography. 
Bruce, A. D. (1980). Adv. Phys. 29, 111-217.

Campbell, B. J., Evans, J. S. O., Perselli, F. \& Stokes, H. T. (2007). CompComm Newsl. 8, 81.

Campbell, B. J., Stokes, H. T., Tanner, D. E. \& Hatch, D. M. (2006). J. Appl. Cryst. 39, 607-614.

Capillas, C., Kroumova, E., Aroyo, M. I., Perez-Mato, J. M., Stokes, H. T. \& Hatch, D. M. (2003). J. Appl. Cryst. 36, 953-954.

Car, R. \& Parrinello, M. (1985). Phys. Rev. Lett. 55, 2471-2474.

Carpenter, M. A. \& Howard, C. J. (2009a). Acta Cryst. B65, 134-146. Carpenter, M. A. \& Howard, C. J. (2009b). Acta Cryst. B65, 147-159.

Catalan, G. \& Scott, J. F. (2009). Adv. Mater. 21, 2463-2485.

Cracknell, A. P., Davies, B. L., Miller, S. C. \& Love, W. F. (1979). Kronecker Product Tables. General Introduction and Tables of Irreducible Representations of Space Groups, Vol. 1. New York: IFI/ Plenum.

Cussen, E. J. \& Battle, P. D. (2000). Chem. Mater. 12, 831-838.

Darlington, C. N. W. (2002). Acta Cryst. A58, 66-71.

Degtyareva, O., McMahon, M. I., Allan, D. R. \& Nelmes, R. J. (2004). Phys. Rev. Lett. 93, 205502.

Dove, M. T. (1993). Introduction to Lattice Dynamics. Cambridge University Press.

Dove, M. T. (1997). Am. Mineral. 82, 213-244.

Dvorak, V. (1971). Phys. Status Solidi B, 45, 147-152.

Fennie, C. J. \& Rabe, K. M. (2005). Phys. Rev. B, 72, 100103.

Fink, H. \& Selfert, H.-J. (1982). Acta Cryst. B38, 912-914.

Fu, W. T. \& Ijdo, D. J. W. (2006). J. Solid State Chem. 179, 27322738.

Giddy, A. P., Dove, M. T., Pawley, G. S. \& Heine, V. (1993). Acta Cryst. A49, 697-703.

Glazer, A. M. (1972). Acta Cryst. B28, 3384-3392.

Hahn, Th. (2002). International Tables for Crystallography, Vol. A. Dordrecht: Kluwer.

Hammonds, K. D., Dove, M. T., Giddy, A. P., Heine, V. \& Winkler, B. (1996). Am. Mineral. 81, 1057-1079.

Hatch, D. M. \& Stokes, H. T. (1985). Phys. Rev. B, 31, 2908-2912.

Hatch, D. M. \& Stokes, H. T. (2001). Phys. Rev. B, 65, 014113.

Haumont, R., Kornev, I. A., Lisenkov, S., Bellaiche, L., Kreisel, J. \& Dkhil, B. (2008). Phys. Rev. B, 78, 134108.

Hendrikse, Z. W. \& Maaskant, W. J. A. (1997). Physica B, 233, 139152.

Hervoches, C. H. \& Lightfoot, P. (1999). Chem. Mater. 11, 3359-3364.

Howard, C. J., Knight, K. S., Kennedy, B. J. \& Kisi, E. H. (2000). J. Phys. Condens. Matter, 12, L677.

Howard, C. J. \& Stokes, H. T. (1998). Acta Cryst. B54, 782-789.

Izyumov, Y. A. \& Syromyatnikov, V. N. (1990). Phase Transitions and Crystal Symmetry. Dordrecht: Kluwer.

Janovec, V. \& Privratska, J. (2003). International Tables for Crystallography, Vol. D, ch. 3.4. Dordrecht: Kluwer.

Janssen, T., Janner, A., Looijenga-Vos, A. \& de Wolff, P. M. (2004). International Tables for Crystallography, Vol. C, ch. 9.8. Dordrecht: Kluwer.

Jaric, M. V. \& Senechal, M. (1984). J. Math. Phys. 25, 3148-3154.

Jeitschko, W. (1972). Acta Cryst. B28, 60-76.

Jongen, L., Gloger, T., Beekhuizen, J. \& Meyer, G. (2005). Z. Anorg. Allg. Chem. 631, 582-586.

Jouini, N., Guen, L. \& Tournoux, M. (1982). Mater. Res. Bull. 17, $1421-1427$

Kennedy, B. J., Howard, C. J. \& Chakoumakos, B. C. (1999). Phys. Rev. B, 59, 4023-4027.

Kennedy, B. J., Prodjosantoso, A. K. \& Howard, C. J. (1999). J. Phys. Condens. Matter, 11, 6319.

Knight, K. S. (2009). Can. Mineral. 47, 381-400.

Koch, E. \& Fischer, W. (2006). Z. Kristrallogr. 221, 1-14.

Kornev, I. A. \& Bellaiche, L. (2009). Phys. Rev. B, 79, 100105.

Kornev, I. A., Lisenkov, S., Haumont, R., Dkhil, B. \& Bellaiche, L. (2007). Phys. Rev. Lett. 99, 227602.

Kwei, G. H., Lawson, A. C., Billinge, S. J. L. \& Cheong, S. W. (1993). J. Phys. Chem. 97, 2368-2377.
Landau, L. D. \& Lifshitz, E. M. (1969). Statistical Physics. Pergamon Press.

Lange, R. A., Carmichael, I. S. E. \& Stebbins, J. F. (1986). Am. Mineral. 71, 937-945.

Lines, M. E. \& Glass, A. M. (1977). Principles and Applications of Ferroelectrics and Related Materials. Oxford University Press.

Lonkai, Th., Tomuta, D. G., Amann, U., Ihringer, J., Hendrikx, R. W. A., Többens, D. M. \& Mydosh, J. A. (2004). Phys. Rev. B, 69 , 134108.

Machida, K.-I., Mitsui, T., Kato, T. \& Katsunori, I. (1994). Solid State Commun. 91, 17-20.

Mañes, J. L., Tello, M. J. \& Perez-Mato, J. M. (1982). Phys. Rev. B, 26, 250-268.

Milman, V., Winkler, B., White, J. A., Pickard, C. J., Payne, M. C., Akhmatskaya, E. V. \& Nobes, R. H. (2000). Int. J. Quant. Chem. 77, 895-910.

Minkiewicz, V. J., Cox, D. E. \& Shirane, G. (1970). Solid State Commun. 8, 1001-1005.

Mitsui, T., Nasui, M., Morishita, K., Kato, T. \& Iio, K. (2000). Ferroelectrics, 238, 97-104.

Morishita, K., Iio, K., Mitsui, T. \& Kato, T. (2001). J. Magn. Magn. Mater. 226-230, 579-581.

Moudden, A. H., Denoyer, F., Lambert, M. \& Fitzgerald, W. (1979). Solid State Commun. 32, 933-936.

Nénert, G., Pollet, M., Marinel, S., Blake, G. R., Meetsma, A. \& Palstra, T. T. M. (2007). J. Phys. Condens. Matter, 19, 466212.

Nishiwaki, Y., Kato, T., Oohara, Y., Oosawa, A., Todoroki, N., Igawa, N., Ishii, Y. \& Iio, K. (2006). J. Phys. Soc. Jpn, 75, 034707.

Oganov, A. R. \& Glass, C. W. (2006). J. Chem. Phys. 124, 244704.

Orobengoa, D., Capillas, C., Aroyo, M. I. \& Perez-Mato, J. M. (2009). J. Appl. Cryst. 42, 820-833.

Palmer, D. C., Dove, M. T., Ibberson, R. M. \& Powell, B. M. (1997). Am. Mineral. 82, 16-29.

Parisi, F. \& Bonadeo, H. (1997). Acta Cryst. A53, 286-290.

Perez-Mato, J. M. (1991). Methods of Structural Analysis of Modulated Structures and Quasicrystals, pp. 117-128. Singapore: World Scientific.

Perez-Mato, J. M., Aroyo, M. I., García, A., Blaha, P., Schwarz, K., Schweifer, J. \& Parlinski, K. (2004). Phys. Rev. B, 70, 214111.

Perez-Mato, J. M., Blaha, P., Schwarz, K., Aroyo, M., Orobengoa, D., Etxebarria, I. \& García, A. (2008). Phys. Rev. B, 77, 184104.

Perez-Mato, J. M., Elcoro, L., Aroyo, M. I., Katzke, H., Tolédano, P. \& Izaola, Z. (2006). Phys. Rev. Lett. 97, 115501.

Perez-Mato, J. M., Gaztelua, F., Madariaga, G. \& Tello, M. J. (1986). J. Phys. C, 19, 1923.

Perez-Mato, J. M., Madariaga, G. \& Tello, M. J. (1984). Phys. Rev. B, 30, 1534-1543.

Perez-Mato, J. M., Madariaga, G., Zuñiga, F. J. \& Garcia Arribas, A. (1987). Acta Cryst. A43, 216-226.

Perez-Mato, J. M., Orobengoa, D., Aroyo, M. I. \& Elcoro, L. (2010). J. Phys. Conf. Ser. 226, 012011.

Perez-Mato, J. M., Withers, R. L., Larsson, A.-K., Orobengoa, D. \& Liu, Y. (2009). Phys. Rev. B, 79, 064111.

Petricek, V., Dusek, M. \& Palatinus, L. (2006). Jana2006 - The Crystallographic Computing System. Institute of Physics, Praha, Czech Republic.

Rabe, K. M., Ahn, C. H. \& Triscone, J.-M. (2007). Physics of Ferroelectrics: A Modern Perspective. Berlin, Heidelberg: Springer Verlag.

Rae, A. D., Thompson, J. G. \& Withers, R. L. (1992). Acta Cryst. B48, 418-428.

Rae, A. D., Thompson, J. G., Withers, R. L. \& Willis, A. C. (1990). Acta Cryst. B46, 474-487.

Rijnsdorp, J. \& Jellinek, F. (1978). J. Solid State Chem. 25, $325-$ 328.

Ritter, H., Ihringer, J., Maichle, J. K. \& Prandl, W. (1998). SIMREF, http://www.uni-tuebingen.de/uni/pki/simref/simref.html.

Rodríguez-Carvajal, J. (1993). Physica B, 192, 55-69. 
Rodríguez-Carvajal, J., Hennion, M., Moussa, F., Moudden, A. H., Pinsard, L. \& Revcolevschi, A. (1998). Phys. Rev. B, 57, R3189R3192.

Selbach, S. M., Tybell, T., Einarsrud, M.-A. \& Grande, T. (2008). Adv. Mater. 20, 3692-3696.

Sikora, W., Białas, F. \& Pytlik, L. (2004). J. Appl. Cryst. 37, 1015-1019.

Smaalen, S. van (1988). Phys. Rev. B, 38, 9594-9600.

Stokes, H. T., Campbell, B. J. \& Hatch, D. M. (2007). Acta Cryst. A63, 365-373.

Stokes, H. T. \& Hatch, D. M. (1988). Isotropy Subgroups of the 230 Crystallographic Space Groups. Singapore: World Scientific.

Stokes, H. T. \& Hatch, D. M. (2002). ISOTROPY, http://stokes. byu.edu/isotropy.html.

Stokes, H. T., Hatch, D. M. \& Wells, J. D. (1991). Phys. Rev. B, 43, 11010-11018.

Tanisaki, S., Mashiyama, H. \& Hasebe, K. (1988). Acta Cryst. B44, 441-445.

Toledano, J. C. \& Toledano, P. (1987). The Landau Theory of Phase Transitions. Singapore: World Scientific.

Tomaszewski, P. E. (1992). Phase Transit. B, 38, 127-220.

Vanderbilt, D. \& Cohen, M. H. (2001). Phys. Rev. B, 63, 094108.
Visser, D., Verschoor, G. C. \& IJdo, D. J. W. (1980). Acta Cryst. B36, 28-34.

Wadhawan, V. K. (2000). Introduction to Ferroic Materials. New York: Gordon and Breach.

Wills, A. S. (2000). Physica B, 276-278, 680-681.

Wondratschek, H. (1993). Contrib. Mineral. Petrol. 48, 87-96.

Woodward, P. M. (1997a). Acta Cryst. B53, 32-43.

Woodward, P. M. (1997b). Acta Cryst. B53, 44-66.

Yamada, N., Ono, Y. \& Ikeda, T. (1984). J. Phys. Soc. Jpn, 53, 25652574.

Yamanaka, K., Nishiwaki, Y., Iio, K., Kato, T., Mitsui, T., Tojo, T. \& Atake, T. (2002). J. Therm. Anal. Calorim. 70, 371-378.

Yin, M. T. \& Cohen, M. L. (1982). Phys. Rev. B, 26, 5668-5687.

Zandbergen, H. W. (1981). J. Solid State Chem. 37, 308-317.

Zhong, W. \& Vanderbilt, D. (1995). Phys. Rev. Lett. 74, 2587-2590.

Zhong, W., Vanderbilt, D. \& Rabe, K. M. (1995). Phys. Rev. B, 52, 6301-6312.

Zhou, Q., Kennedy, B. J. \& Howard, C. J. (2003). Chem. Mater. 15, 5025-5028.

Zuñiga, F. J., Madariaga, G., Paciorek, W. A., Pérez-Mato, J. M., Ezpeleta, J. M. \& Etxebarria, I. (1989). Acta Cryst. B45, 566-576. 